\title{
MODULARITY LIFTING RESULTS IN PARALLEL WEIGHT ONE AND APPLICATIONS TO THE ARTIN CONJECTURE: THE TAMELY RAMIFIED CASE
}

\author{
PAYMAN L. KASSAEI ${ }^{1}$, SHU SASAKI ${ }^{2}$ and YICHAO TIAN ${ }^{3}$ \\ ${ }^{1}$ Department of Mathematics and Statistics, McGill University, 805 Sherbrooke St. W., \\ Montreal H3A 0B9, QC, Canada; \\ email: kassaei@math.mcgill.ca \\ ${ }^{2}$ Fakultat fur Mathematik, Universitat Duisburg-Essen, Thea-Leymann-Strasse 9, \\ 45127 Essen, Germany; \\ email: s.sasaki.03@cantabgold.net \\ ${ }^{3}$ Morningside Center of Mathematics, Chinese Academy of Sciences, \\ 55 Zhong Guan Cun East Road, Beijing, 100190, China; \\ email: yichaot@math.ac.cn
}

Received 20 May 2013; accepted 6 May 2014

\begin{abstract}
We extend the modularity lifting result of P. Kassaei ('Modularity lifting in parallel weight one', J. Amer. Math. Soc. 26 (1) (2013), 199-225) to allow Galois representations with some ramification at $p$. We also prove modularity mod 5 of certain Galois representations. We use these results to prove new cases of the strong Artin conjecture over totally real fields in which 5 is unramified. As an ingredient of the proof, we provide a general result on the automatic analytic continuation of overconvergent $p$-adic Hilbert modular forms of finite slope which substantially generalizes a similar result in P. Kassaei ('Modularity lifting in parallel weight one', J. Amer. Math. Soc. 26 (1) (2013), 199-225).
\end{abstract}

2010 Mathematics Subject Classification: 11F33 (primary); 11F41, 11F80 (secondary)

\section{Introduction}

The work of Buzzard and Taylor [6] beautifully combined methods of Wiles and Taylor (à la Diamond [11]) with a geometric analysis of overconvergent $p$-adic modular forms to prove a modularity lifting result for geometric representations of $\operatorname{Gal}(\overline{\mathbb{Q}} / \mathbb{Q})$ which are split and unramified at $p$. Combined with the works of

(C) The Author(s) 2014. The online version of this article is published within an Open Access environment subject to the conditions of the Creative Commons Attribution licence $<$ http://creativecommons.org/licenses/by/3.0/>. 
Shepherd-Barron, Taylor, and Dickinson, these ideas came together in [5], where a program laid out by Taylor came to fruition in proving many cases of the Artin conjecture over $\mathbb{Q}$. Later, Buzzard generalized the results of [6], allowing the Galois representation to have ramification at $p$, and these results were used by Taylor to prove more cases of the Artin conjecture.

In [19], the first-named author proved a generalization of the main result of Buzzard and Taylor [6] over a totally real field in which $p$ is unramified. In [27], Pilloni proved a generalization of this result in the case where $p$ is slightly ramified in $F$. In both works, the Galois representation is assumed unramified at $p$. In this work, we generalize the result of [19] allowing some ramification at $p$ for the Galois representation in question.

To explain our method, let us first give a brief description of the proof in [19]. At the heart of the argument in [19] lies the proof that a collection of weightone specializations of Hida families are all classical Hilbert modular forms by analytically extending them from their initial domains of definition to the entire Hilbert modular variety $\mathfrak{Y}_{\text {rig }}$ of level $\Gamma_{1}(N) \cap \Gamma_{0}(p)$ (where $N$ is prime to $p$ ). This analytic continuation is done in two steps. First, one proves that every finite slope overconvergent Hilbert modular form will automatically extend to a region $\mathcal{R}$ inside $\mathfrak{Y}_{\text {rig }}$, a region considerably larger than the locus of definition of the canonical subgroup, $\mathcal{V}_{\text {can }}$, over which one could previously perform automatic analytic continuation (see [15]). In the second step, using a gluing argument, one shows that a linear combination of the weight-one forms at hand extends to a yet bigger region $\mathcal{R} \subset \mathfrak{Y}_{\text {rig }}^{|\tau| \leqslant 1} \subset \mathfrak{Y}_{\text {rig }}$ which contains a region saturated with respect to the forgetful map $\pi: \mathfrak{Y}_{\text {rig }} \rightarrow \mathfrak{X}_{\text {rig }}$ to the Hilbert modular variety $\mathfrak{X}_{\text {rig }}$ of level $\Gamma_{1}(N)$. It is, then, proved that this linear combination descends under the map $\pi$ to a region $\mathfrak{X}_{\text {rig }}^{|\tau| \leqslant 1}=\pi\left(\mathfrak{Y}_{\text {rig }}^{|\tau| \leqslant 1}\right) \subseteq \mathfrak{X}_{\text {rig }}$, over which a rigid-analytic Koecher principle can be applied to extend the descended form to the entire $\mathfrak{X}_{\text {rig }}$. The point is that, while $\mathcal{R}$ and $\mathfrak{Y}_{\text {rig }}^{|\tau| \leqslant 1}$ are not large enough to make the application of the Koecher principle possible, their images under $\pi$ are.

In the setting of this article, we can no longer descend the forms to level $\Gamma_{1}(N)$, and, hence, we must prove a more optimal analytic continuation at the level of $\mathfrak{Y}_{\text {rig }}$ itself. In this work, we prove that every finite slope overconvergent Hilbert modular form extends to a region $\Sigma$ which is vastly larger than $\mathcal{R}$, a region only slightly smaller than the tube of the 'generic' locus of the special fibre $\bar{Y}$ (in codimension $<2$ ), defined in terms of the stratification of $\bar{Y}$ studied in [15].

THEOREM 1.1. Any overconvergent Hilbert modular form of finite slope (at all primes above $p$ ) extends analytically to

$$
\left.\Sigma=\bigcup_{\operatorname{codim}(W)=0,1}\right] W^{\text {gen }} \Gamma^{\prime},
$$


where $W$ runs over strata of $\bar{Y}$ (defined in [15]) of codimension $0,1, W^{\text {gen }}$ is the generic locus of $W$ defined in Definition 2.5, $\left(W^{\text {gen }}\right)$ is the tube of $W^{\text {gen }}$ in $\mathfrak{Y}_{\text {rig, }}$, and $] W^{\text {gen }}\left[{ }^{\prime} \subset\left(W^{\text {gen }}\right)\right.$ is an admissible open subset defined in Definition 5.6.

This provides the strongest automatic analytic continuation result for overconvergent Hilbert modular forms in the literature so far, and is of independent interest besides the applications explored in this article. This is done in Section 5.

To explain the second step of the proof, let us assume that the prime $p$ is inert in $F$, for simplicity. In this case, the collection of weight-one overconvergent forms consists of two forms, say $f, g$, each of which can be extended to $\Sigma$ by the first step. Let $w$ be the Atkin-Lehner involution on $\mathfrak{Y}_{\text {rig. }}$. To further extend $f$, we want to glue $f$ defined on $\Sigma$ to a scalar multiple of $w(g)$ which is defined on $w^{-1}(\Sigma)$. This would extend $f$ to $\Sigma \cup w^{-1}(\Sigma)$. The main difficulty in this step, compared to the elliptic case, is that $\Sigma \cap w^{-1}(\Sigma)$ has many connected components which need to be controlled. A detailed analysis of these connected components is carried out in Section 6; it utilizes the geometry of intersection of irreducible components of strata on $\bar{Y}$ provided by [15]. Another complication, essentially due to the existence of totally positive units in $F$, is that $f$ may, in fact, not be a scalar multiple of $w(g)$, and a replacement for $g$ has to be constructed.

The third step of the analytic continuation requires a close study of the stratification on $\bar{Y}$, which is carried out in Section 2. Using these results, one shows that the complement of $\Sigma \cup w^{-1}(\Sigma)$ in $\mathfrak{Y}_{\text {rig }}$ has codimension at least 2 in reduction mod $p$. It follows that $f$ automatically extends from $\Sigma \cap w^{-1}(\Sigma)$ to the entire $\mathfrak{Y}_{\text {rig }}$ by the Koecher principle. We should mention that there are smaller choices of $\Sigma$ that make this last step work fine. However, the region $\Sigma$ defined above is chosen such that the connected components of $\Sigma \cap w^{-1}(\Sigma)$ are well behaved: we show that every such connected component contains a region which is saturated under $\pi$; this is essential for carrying out the gluing process described above. The above analytic continuation results allow us to prove the following.

THEOREM 1.2. Let $p>2$ be a prime number, and $F$ a totally real field in which $p$ is unramified. For any prime ideal $\mathfrak{p} \mid p$, let $D_{\mathfrak{p}}$ denote a decomposition group of $\operatorname{Gal}(\overline{\mathbb{Q}} / F)$ at $\mathfrak{p}$, and $I_{\mathfrak{p}}$ the inertia subgroup. Let $\rho: \operatorname{Gal}(\overline{\mathbb{Q}} / F) \rightarrow \mathrm{GL}_{2}(\mathcal{O})$ be a continuous representation, where $\mathcal{O}$ is the ring of integers in a finite extension of $\mathbb{Q}_{p}$, and $\mathfrak{m}$ its maximal ideal. Assume the following.

- $\rho$ is unramified outside a finite set of primes.

- For every prime $\mathfrak{p} \mid p$, we have

$$
\rho_{\left.\right|_{\mathfrak{p}}} \cong \alpha_{\mathfrak{p}} \oplus \beta_{\mathfrak{p}}
$$


where $\alpha_{\mathfrak{p}}, \beta_{\mathfrak{p}}: D_{\mathfrak{p}} \rightarrow \mathcal{O}^{\times}$are characters distinct modulo $\mathfrak{m}$, and $\alpha_{\mathfrak{p}}\left(I_{\mathfrak{p}}\right)$ and $\beta_{\mathfrak{p}}\left(I_{\mathfrak{p}}\right)$ are finite, and $\alpha_{\mathfrak{p}} / \beta_{\mathfrak{p}}$ is tamely ramified.

- $\bar{\rho}:=(\rho \bmod \mathfrak{m})$ is ordinarily modular; that is, there exists a classical Hilbert modular form $g$ of parallel weight 2 such that $\rho \equiv \rho_{g}(\bmod \mathfrak{m})$ and $\rho_{g}$ is potentially ordinary and potentially Barsotti-Tate at every prime of $F$ dividing $p$.

- $\bar{\rho}$ is absolutely irreducible when restricted to $\mathrm{Gal}\left(\overline{\mathbb{Q}} / F\left(\zeta_{p}\right)\right)$.

Then, $\rho$ is isomorphic to $\rho_{f}$, the Galois representation associated to a Hilbert modular eigenform $f$ of weight $(1,1, \ldots, 1)$ and level $\Gamma_{1}(N p)$, for some integer $N$ prime to $p$.

REMARK 1.3 . We can handle the case where $\alpha_{\mathfrak{p}} / \beta_{\mathfrak{p}}$ has arbitrary ramification provided there is an integral model, not necessarily having any moduli interpretation, of the Hilbert modular variety of level $\Gamma_{1}\left(N p^{m}\right)$ (for $m>1$ ) such that the forgetful map to level $\Gamma_{1}(N p)$ is flat.

In the last two sections of the paper, we apply our results to prove certain cases of the strong Artin conjecture over totally real fields. We first prove modularity of certain Galois representations mod 5 following Taylor [42]. Combining these results with Theorem 1.2, we prove new icosahedral cases of the strong Artin conjecture over $F$ (generalizing the main theorem of Taylor in [42] to $F$ while removing conditions at 3 and 5 given there).

THEOREM 1.4. Let $F$ be a totally real field in which 5 is unramified. Let

$$
\rho: \operatorname{Gal}(\overline{\mathbb{Q}} / F) \rightarrow \mathrm{GL}_{2}(\mathbb{C})
$$

be a totally odd and continuous representation satisfying the following conditions:

- $\rho$ is totally odd;

- $\rho$ has the projective image $A_{5}$;

- for every place $\mathfrak{p}$ of $F$ above 5, the projective image of the decomposition group at $\mathfrak{p}$ has order 2.

Then, there exists a holomorphic Hilbert cuspidal eigenform $f$ of weight 1 such that $\rho$ arises from $f$ in the sense of Rogawsi and Tunnell, and the Artin L-function $L(\rho, s)$ is entire. 
After the first version of this article was submitted to Arxiv, Pilloni and Stroh [30] announced an unconditional proof of the strong Artin conjecture for degree two totally odd Galois representations over $F$.

1.5. Notation. Let $p$ be a prime number. Let $L / \mathbb{Q}$ be a totally real field of degree $g>1$ in which $p$ is unramified, $\mathcal{O}_{L}$ its ring of integers, and $\mathfrak{d}_{L}$ its different ideal. For a prime ideal $\mathfrak{p}$ of $\mathcal{O}_{L}$ dividing $p$, let $\kappa_{\mathfrak{p}}=\mathcal{O}_{L} / \mathfrak{p}$, a finite field of order $p^{f_{\mathfrak{p}}}$. Let $\kappa$ be a finite field containing an isomorphic copy of all $\kappa_{\mathfrak{p}}$ and generated by their images. We identify $\kappa_{\mathfrak{p}}$ with a subfield of $\kappa$ once and for all. Let $\mathbb{Q}_{\kappa}$ be the fraction field of $W(\kappa)$. We fix embeddings $\mathbb{Q}_{\kappa} \subset \mathbb{Q}_{p}^{\text {ur }} \subset \overline{\mathbb{Q}}_{p}$. Let $v_{p}$ be the $p$-adic valuation on $\overline{\mathbb{Q}}_{p}$ so that $v_{p}(p)=1$.

Let $\mathbb{S}=\{\mathfrak{p} \mid p\}$ be the set of prime ideals of $\mathcal{O}_{L}$ dividing $p$. Let

$$
\mathbb{B}=\operatorname{Emb}\left(L, \mathbb{Q}_{\kappa}\right)=\bigsqcup_{\mathfrak{p} \in \mathbb{S}} \mathbb{B}_{\mathfrak{p}},
$$

where $\mathbb{B}_{\mathfrak{p}}=\left\{\beta \in \mathbb{B}: \beta^{-1}(p W(\kappa))=\mathfrak{p}\right\}$, for every prime ideal $\mathfrak{p}$ dividing $p$. If $\mathfrak{t} \mid(p)$ is an ideal, we define $\mathbb{B}_{\mathfrak{t}}=\cup_{\mathfrak{p} \mid \mathfrak{t}} \mathbb{B}_{\mathfrak{p}}$. We also set $\mathfrak{t}^{*}=(p) / \mathfrak{t}$.

Let $\sigma$ denote the Frobenius automorphism of $\mathbb{Q}_{\kappa}$, lifting $x \mapsto x^{p}$ on $\kappa$. It acts on $\mathbb{B}$ via $\beta \mapsto \sigma \circ \beta$, and transitively on each $\mathbb{B}_{\mathfrak{p}}$. For $S \subseteq \mathbb{B}$, we let $S^{c}=\mathbb{B}-S$, and $\ell(S)=\left\{\sigma^{-1} \circ \beta: \beta \in S\right\}$, the left shift of $S$. Similarly, define the right shift of $S$, denoted $r(S)$. We denote by $|S|$ the cardinality of $S$. The decomposition

$$
\mathcal{O}_{L} \otimes_{\mathbb{Z}} W(\kappa)=\bigoplus_{\beta \in \mathbb{B}} W(\kappa)_{\beta},
$$

where $W(\kappa)_{\beta}$ is $W(\kappa)$ with the $\mathcal{O}_{L}$-action given by $\beta$, induces a decomposition,

$$
M=\bigoplus_{\beta \in \mathbb{B}} M_{\beta}
$$

on any $\mathcal{O}_{L} \otimes_{\mathbb{Z}} W(\kappa)$-module $M$.

Let $N \geqslant 4$ be an integer prime to $p$. Throughout the paper, we will fix a finite extension $K$ of $\mathbb{Q}_{\kappa}$ with residue field $\kappa_{K}$, and ring of integers $\mathcal{O}_{K}$.

1.6. The Hilbert modular variety of level $\Gamma_{1}(N) \cap \boldsymbol{\Gamma}_{\mathbf{0}}(p)$. We denote by $X$ the Hilbert modular scheme of level $\Gamma_{1}(N)$ over $\operatorname{Spec}\left(\mathcal{O}_{K}\right)$, and by $Y$ the Hilbert modular scheme over $\operatorname{Spec}\left(\mathcal{O}_{K}\right)$ of level $\Gamma_{1}(N) \cap \Gamma_{0}(p)$. The base extensions of $X$ and $Y$ to $\operatorname{Spec}(K)$ are denoted by $X_{K}$ and $Y_{K}$, respectively. The connected components of $X$ and $Y$ are both in natural bijection with the set of strict ideal class group $c l_{L}^{+}$. For a representative $\left(\mathfrak{a}, \mathfrak{a}^{+}\right)$of $c l_{L}^{+}$, we denote the corresponding 
connected component of $X$ (respectively, $Y$ ) by $X_{\mathfrak{a}}$ (respectively, $Y_{\mathfrak{a}}$ ). We apply the same convention to other variants of $X$ and $Y$ appearing in this paper. Let $\bar{X}$ and $\bar{Y}$ be, respectively, the special fibres of $X$ and $Y, \mathfrak{X}$ and $\mathfrak{Y}$ the formal completions of $X$ and $Y$ along their special fibres, and $\mathfrak{X}_{\text {rig }}$ and $\mathfrak{Y}_{\text {rig }}$ the associated rigid analytic generic fibres over $K$. By abuse of notation, we always denote by $\pi$ the natural forgetful projection from level $\Gamma_{1}(N) \cap \Gamma_{0}(p)$ to $\Gamma_{1}(N)$ in various settings, for example, $\pi: Y \rightarrow X, \pi: \mathfrak{Y} \rightarrow \mathfrak{X}, \pi: \mathfrak{Y}_{\text {rig }} \rightarrow \mathfrak{X}_{\text {rig. }}$.

Let $\mathcal{A}^{\text {univ }}$ be the universal abelian scheme over $X$, and $e: X \rightarrow \mathcal{A}^{\text {univ }}$ be the unit section. We put $\omega=e^{*} \Omega_{\mathcal{A}^{\text {univ }} / X}$. This is a locally free $\left(\mathcal{O}_{X} \otimes_{\mathbb{Z}} \mathcal{O}_{L}\right)$-module of rank 1, so that we have a canonical decomposition $\omega=\oplus_{\beta \in \mathbb{B}} \omega_{\beta}$ according to the natural action of $\mathcal{O}_{L}$ on $\omega$. For any $\underline{k}=\left(k_{\beta}\right)_{\beta \in \mathbb{B}} \in \mathbb{Z}^{\mathbb{B}}$, we put

$$
\omega^{\underline{k}}=\otimes_{\beta \in \mathbb{B}} \omega_{\beta}^{\otimes k_{\beta}}
$$

We still denote by $\omega, \omega_{\beta}$ and $\omega^{k}$ their pull-backs to $Y$ via $\pi$ or the corresponding sheaves in the formal or rigid analytic settings.

We choose toroidal compactifications $\tilde{X}$ and $\tilde{Y}$ based on a common fixed choice of rational polyhedral cone decompositions. We still denote by $\pi$ the natural map $\tilde{Y} \rightarrow \tilde{X}$. There is a semi-abelian scheme $\tilde{\mathcal{A}}$ over $\tilde{X}$ extending the universal abelian scheme $\mathcal{A}^{\text {univ }}$. We denote by $\tilde{\mathfrak{X}}$ and $\tilde{\mathfrak{Y}}$ the corresponding formal completions along the special fibres, and by $\tilde{\mathfrak{X}}_{\text {rig }}=\tilde{X}_{K}^{\text {an }}$ and $\tilde{\mathfrak{Y}}_{\text {rig }}=\tilde{Y}_{K}^{\text {an }}$ the associated rigid analytic spaces. For any $\underline{k} \in \mathbb{Z}^{\mathbb{B}}$, the line bundle $\omega^{\underline{k}}$ on $X$ (respectively, on $Y$ ) extends to a line bundle over $\tilde{X}$ (respectively, $\tilde{Y}$ ), still denoted by $\omega^{\underline{k}}$, by using the semi-abelian scheme $\tilde{\mathcal{A}}$ over $\tilde{X}$ (respectively, $\tilde{Y}$ ).

Let $\tilde{\mathfrak{X}}_{\text {rig }}^{\text {ord }}$ be the ordinary locus of $\mathfrak{X}_{\text {rig }}$, that is, the quasi-compact admissible open subdomain of $\tilde{\mathfrak{X}}_{\text {rig }}$, where the rigid semi-abelian scheme $\tilde{\mathcal{A}}^{\text {an }}$ has good ordinary reduction or specializes to the cusps, and $\mathfrak{X}_{\text {rig }}^{\text {ord }}=\tilde{\mathfrak{X}}_{\text {rig }}^{\text {ord }} \cap \mathfrak{X}_{\text {rig }}$. Similarly, let $\mathfrak{Y}_{\text {rig }}^{\text {ord }}$ be the locus of $\pi^{-1}\left(\mathfrak{X}_{\text {rig }}^{\text {ord }}\right)$ where the finite flat subgroup $H$ is of multiplicative

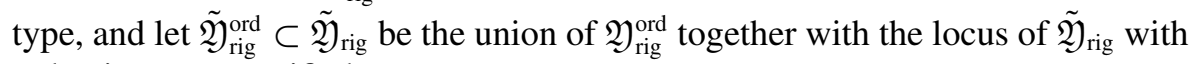
reduction to unramified cusps.

1.7. The Hilbert modular variety of level $\Gamma_{1}(N p)$. Recall our fixed choice of $K$, a finite extension of $\mathbb{Q}_{\kappa}$. We assume that $K$ contains the $p$ th roots of unity. Let $Z_{K}$ be the Hilbert modular variety of level $\Gamma_{1}(N p)$ over $\operatorname{Spec}(K)$, that is, the scheme that represents the functor attaching to a $K$-scheme $S$, the set of isomorphic classes of the 4-tuples, $\left(A, \lambda, i_{N}, P\right)$, where

- $A$ is an abelian scheme of dimension $g$ over $S$ with real multiplication by $\mathcal{O}_{L}$;

- $\lambda: A \rightarrow A^{\vee}$ is a prime-to- $p$ polarization compatible with the action of $\mathcal{O}_{L}$; 
- $i_{N}: \mu_{N} \otimes_{\mathbb{Z}} \mathfrak{d}_{L}^{-1} \hookrightarrow A[N]$ is an $\mathcal{O}_{L}$-equivariant closed immersion of group schemes;

- $P: S \rightarrow A[p]$ is a section of the finite flat group scheme $A[p]$ of order $p$, such that the $\mathcal{O}_{L}$-subgroup generated by $P$ is a free $\left(\mathcal{O}_{L} / p\right)$-module of rank one.

We will often use the abbreviation $\underline{A}=\left(A, \lambda, i_{N}\right)$, and denote an $S$-valued point of $Z_{K}$ simply by $(\underline{A}, P)$. Let $H=(P)$ be the $\mathcal{O}_{L}$-subgroup of $A[p]$ generated by $P$. We have a canonical decomposition $H=\prod_{\mathfrak{p} \in \mathbb{S}} H[\mathfrak{p}]$ corresponding to the decomposition $\mathcal{O}_{L} / p=\prod_{\mathfrak{p} \in \mathbb{S}} \mathcal{O}_{L} / \mathfrak{p}$. We denote by $P_{\mathfrak{p}}$ the image of $P$ under the natural projection $H \rightarrow H[\mathfrak{p}]$. Then $P_{\mathfrak{p}}$ is a generator of $H[\mathfrak{p}]$ as $\mathcal{O}_{L}$-module, and we have $P=\prod_{\mathfrak{p} \in \mathbb{S}} P_{\mathfrak{p}}$. Let

$$
\alpha: Z_{K} \rightarrow Y_{K}
$$

be the map given by $(\underline{A}, P) \mapsto(\underline{A},(P))$. The map $\alpha$ is finite étale of degree $\prod_{\mathfrak{p} \in \mathbb{S}}\left(p^{f_{\mathfrak{p}}}-1\right)$.

We choose the same rational polyhedral cone decomposition as that of $Y$ to construct a toroidal compactification $\tilde{Z}_{K}$ of $Z_{K}$. The morphism $\alpha$ extends to a finite flat map $\alpha: \tilde{Z}_{K} \rightarrow \tilde{Y}_{K}$. For any $\underline{k}=\left(k_{\beta}\right)_{\beta \in \mathbb{B}} \in \mathbb{Z}^{\mathbb{B}}$, we define $\omega^{\underline{k}}$ on $\tilde{Z}_{K}$ to be the pullback of $\omega^{\underline{k}}$ on $\tilde{Y}_{K}$ under $\alpha$.

For uniformity of notation, we will denote $\tilde{Z}_{K}^{\text {an }}$, the rigid analytification of $\tilde{Z}_{K}$, by $\tilde{\mathfrak{Z}}_{\text {rig. }}$. We will continue to denote by $\alpha: \tilde{\mathfrak{Z}}_{\text {rig }}=\tilde{Z}_{K}^{\text {an }} \rightarrow \tilde{Y}_{K}^{\text {an }}=\tilde{\mathfrak{Y}}_{\text {rig }}$ the analytification of $\alpha: \tilde{Z}_{K} \rightarrow \tilde{Y}_{K}$. We set $\tilde{\mathfrak{Z}}_{\text {rig }}^{\text {ord }}=\alpha^{-1}\left(\tilde{\mathfrak{Y}}_{\text {rig }}^{\text {ord }}\right)$. The analytification of the sheaf $\omega^{\underline{k}}$ on $\tilde{\mathfrak{Z}}_{\text {rig }}$ will be denoted by the same symbol.

1.8. An integral model of $Z_{K}$. We will need the integral model of $Z_{K}$ over $\mathcal{O}_{K}$ defined in [26]. Let $H \subset \mathcal{A}^{\text {univ }}[p]$ be the universal isotropic $\left(\mathcal{O}_{L} / p\right)$-cyclic subgroup over $Y$. We have a canonical decomposition $H=\prod_{\mathfrak{p} \in \mathbb{S}} H[\mathfrak{p}]$, where each $H[\mathfrak{p}]=\operatorname{Spec}\left(\mathcal{O}_{H[\mathfrak{p}]}\right)$ is a scheme of one-dimensional $\left(\mathcal{O}_{L} / \mathfrak{p}\right)$-vector spaces over $Y$. By Raynaud's classification of such group schemes [32, 1.4.1], there exist invertible sheaves $\mathcal{L}_{\beta}$ over $Y$, for each $\beta \in \mathbb{B}_{\mathfrak{p}}$, together with $\mathcal{O}_{Y}$-linear morphisms $\Delta_{\beta}: \mathcal{L}_{\beta}^{\otimes p} \rightarrow \mathcal{L}_{\sigma \circ \beta}$ and $\Gamma_{\beta}: \mathcal{L}_{\sigma \circ \beta} \rightarrow \mathcal{L}_{\beta}^{\otimes p}$ such that $\Delta_{\beta} \circ \Gamma_{\beta}$ and $\Gamma_{\beta} \circ \Delta_{\beta}$ are both multiplication by $p$, and the $\mathcal{O}_{Y}$-algebra $\mathcal{O}_{H[\mathfrak{p}]}$ is isomorphic to

$$
\operatorname{Sym}_{\mathcal{O}_{Y}}\left(\oplus_{\beta \in \mathbb{B}} \mathcal{L}_{\beta}\right) /\left(\left(1-\Delta_{\beta}\right) \mathcal{L}_{\beta}^{\otimes p}: \beta \in \mathbb{B}_{\mathfrak{p}}\right) .
$$

In fact, $\mathcal{L}_{\beta}$ is the direct summand of the augmentation ideal $\mathcal{J}_{H[\mathfrak{p}]} \subset \mathcal{O}_{H[\mathfrak{p}]}$, where $\left(\mathcal{O}_{L} / \mathfrak{p}\right)^{\times}$acts via the Teichmüller character $\chi_{\beta}:\left(\mathcal{O}_{L} / \mathfrak{p}\right)^{\times} \rightarrow W\left(\kappa_{K}\right)^{\times}$. Now we consider the closed subscheme $H^{\prime}[\mathfrak{p}]$ of $H$ [p] defined by the equation

$$
\left(\otimes_{\beta \in \mathbb{B}} \Delta_{\beta}-1\right)\left(\otimes_{\beta \in \mathbb{B}} \mathcal{L}_{\beta}^{\otimes(p-1)}\right) .
$$


Explicitly, let $U=\operatorname{Spec}(R)$ be an affine open subset of $Y$ such that

$$
\left.H[\mathfrak{p}]\right|_{U}=\operatorname{Spec}(R)\left[T_{\beta}: \beta \in \mathbb{B}_{\mathfrak{p}}\right] /\left(T_{\beta}^{p}-y_{\sigma \circ \beta} T_{\sigma \circ \beta}: \beta \in \mathbb{B}_{\mathfrak{p}}\right),
$$

with $y_{\beta} \in R$, for $\beta \in \mathbb{B}_{\mathfrak{p}}$. Then, we have

$$
\left.H^{\prime}[\mathfrak{p}]\right|_{U}=\operatorname{Spec}\left(R\left[T_{\beta}: \beta \in \mathbb{B}_{\mathfrak{p}}\right]\right) /\left(T_{\beta}^{p}-y_{\sigma \circ \beta} T_{\sigma \circ \beta}, \prod_{\beta \in \mathbb{B}_{\mathfrak{p}}} T_{\beta}^{p-1}-\prod_{\beta \in \mathbb{B}_{\mathfrak{p}}} y_{\beta}\right) .
$$

From this local description, we see that $H^{\prime}[\mathfrak{p}]$ is a finite flat scheme over $Y$ of rank $p^{f_{\mathfrak{p}}}-1$. We set $H^{\prime}:=\prod_{\mathfrak{p} \in \mathbb{S}} H^{\prime}[\mathfrak{p}]$.

Proposition 1.9 ([26], 5.1.5, 2.3.3). The $Y$-scheme $H^{\prime} \rightarrow Y$ represents the functor which associates to each $Y$-scheme $S$, the set of $\left(\mathcal{O}_{L} / p\right)$-generators of $H \times_{Y} S$ in the sense of Drinfeld, Katz, and Mazur [21, 1.10]. Consequently, the scheme $H^{\prime}$ is an integral model of $Z$ over $\mathcal{O}_{K}$, which is finite flat of degree $\prod_{\mathfrak{p} \in \mathbb{S}}\left(p^{f_{\mathfrak{p}}}-1\right)$ over $Y$.

In what follows, we will define $Z:=H^{\prime}$, and call $\alpha$ the natural map $Z \rightarrow Y$. For any $\underline{k}$ as above, we let $\omega^{\underline{k}}$ denote the pullback of $\omega^{\underline{k}}$ on $Y$ under $\alpha: Z \rightarrow Y$; it is an integral model for the restriction to $Z_{K}$ of the sheaf $\omega^{k}$ defined above on $\tilde{Z}_{K}$.

We let $\mathfrak{Z}$ denote the formal completion of $Z$ along its special fibre, and $\mathfrak{Z}_{\text {rig }}$ its associated rigid generic fibre. Therefore, $\mathfrak{Z}_{\text {rig }}$ is the quasi-compact admissible open subdomain of $\tilde{\mathfrak{Z}}_{\text {rig }}$ where the universal HBAV has good reduction.

1.10. Atkin-Lehner automorphisms. We first define these automorphisms over $Y$. Let $S$ be an $\mathcal{O}_{K}$-scheme, and consider an $S$-valued point $Q$ of $Y$ corresponding to $(\underline{A}, H)=\left(A, i_{N}, \lambda, H\right)$. We have a canonical decomposition $H=\prod_{\mathfrak{q} \in \mathbb{S}} H[\mathfrak{q}]$. For any $\mathfrak{p} \in \mathbb{S}$, we put

$$
w_{\mathfrak{p}}(Q)=\left(\underline{A} / H[\mathfrak{p}], H^{\prime}\right),
$$

where $\underline{A} / H[\mathfrak{p}]$ denotes the quotient of $A$ by $H[\mathfrak{p}]$ along with its induce PEL data (as defined in [15, Section 2.1]), and $H^{\prime}=\prod_{\mathfrak{q}} H^{\prime}[\mathfrak{q}]$, with $H^{\prime}[\mathfrak{p}]=A[\mathfrak{p}] / H[\mathfrak{p}]$ and $H^{\prime}[\mathfrak{q}]=H[\mathfrak{q}]$, under the identification $(A / H[\mathfrak{p}])[\mathfrak{q}] \cong A[\mathfrak{q}]$ for $\mathfrak{q} \neq \mathfrak{p}$. The automorphisms $w_{\mathfrak{p}}$ for $\mathfrak{p} \in \mathbb{S}$ commute with each other. For any $T \subset \mathbb{S}$, we define $w_{T}=\prod_{\mathfrak{p} \in T} w_{\mathfrak{p}}$, and $w=w_{\mathbb{S}}$. In view of

$$
w^{2}(Q)=\left(\prod_{\mathfrak{p} \in \mathbb{S}} w_{\mathfrak{p}}^{2}\right)(Q)=\left(A, \lambda, p i_{N}, H\right)
$$


we see that each $w_{T}$ is automorphism on $Y$. The automorphisms $w_{T}$ extend naturally to the chosen toroidal compactification $\tilde{Y}$. We still denote by $w_{T}$ the automorphisms induced on $\tilde{Y}_{K}, \tilde{\bar{Y}}, \tilde{\mathfrak{Y}}_{\text {rig }}$, etc.

We now turn to $Z_{K}$. Let us fix an element $\zeta_{\mathfrak{p}}$ which is an $\mathcal{O}_{L}$-generator of $\left(\mathbb{G}_{m} \otimes \mathfrak{d}_{L}^{-1}\right)[\mathfrak{p}]$. We define an automorphism $w_{\mathfrak{p}}$ on $Z_{K}$ for $\mathfrak{p} \in \mathbb{S}$ as follows. Let $x=(\underline{A}, P)$ be a point of $Z_{K}$ with values in a $K$-scheme $S$. For each $\mathfrak{p} \in \mathbb{S}$, the prime-to- $p$ polarization $\lambda$ induces a perfect $\mathcal{O}_{L}$-linear Weil pairing

$$
\langle\cdot, \cdot\rangle_{\mathfrak{p}}: A[\mathfrak{p}] \times A[\mathfrak{p}] \rightarrow\left(\mathbb{G}_{m} \otimes \mathfrak{d}_{L}^{-1}\right)[\mathfrak{p}] .
$$

Let $\phi_{\mathfrak{p}}: A \rightarrow A^{\prime}=A / H[\mathfrak{p}]$ be the canonical isogeny, and let $\hat{\phi}_{\mathfrak{p}}: A^{\prime} \rightarrow A / A[\mathfrak{p}]$ be the canonical isogeny with kernel $A[\mathfrak{p}] / H[\mathfrak{p}]$. Since $H[\mathfrak{p}]$ is (automatically) isotropic, the Weil pairing $\langle\cdot, \cdot\rangle_{\mathfrak{p}}$ induces a perfect duality pairing

$$
\langle\cdot, \cdot\rangle_{\phi_{\mathfrak{p}}}: \operatorname{Ker}\left(\phi_{\mathfrak{p}}\right) \times \operatorname{Ker}\left(\hat{\phi}_{\mathfrak{p}}\right) \rightarrow\left(\mathbb{G}_{m} \otimes \mathfrak{d}_{L}^{-1}\right)[\mathfrak{p}] .
$$

We define $w_{\mathfrak{p}}(x)=\left(\underline{A}^{\prime}, Q\right)=\left(A^{\prime}, \lambda^{\prime}, i_{N}^{\prime}, Q\right)$, where $\lambda^{\prime}$ and $i_{N}^{\prime}$ are respectively the induced polarization and $\Gamma_{1}(N)$-structure on $A^{\prime}$, and $Q=\prod_{\mathfrak{q} \in \mathbb{S}} Q_{\mathfrak{q}} \in A^{\prime}[p](S)$ is given as follows. For $\mathfrak{q} \neq \mathfrak{p}$, we put

$$
Q_{\mathfrak{q}}=P_{\mathfrak{q}} \in A^{\prime}[\mathfrak{q}] \simeq A[\mathfrak{p}],
$$

and $Q_{\mathfrak{p}}$ is the unique point of $\operatorname{Ker}\left(\hat{\phi}_{\mathfrak{p}}\right)(S)$ such that $\left\langle P_{\mathfrak{p}}, Q_{\mathfrak{p}}\right\rangle_{\phi_{\mathfrak{p}}}=\zeta_{\mathfrak{p}}$.

We see easily that $w_{\mathfrak{p}}$ and $w_{\mathfrak{q}}$ commute for $\mathfrak{p}, \mathfrak{q} \in \mathbb{S}$, so that $w_{T}=\prod_{\mathfrak{p} \in T} w_{\mathfrak{p}}$ is well defined for any $T \subset \mathbb{S}$. We let $w=w_{\mathbb{S}}$. Note that $w^{2}\left(A, \lambda, i_{N}, P\right)=(A, \lambda$, $\left.p i_{N},-P\right)$, since

$$
\left\langle P_{\mathfrak{p}}, Q_{\mathfrak{p}}\right\rangle_{\phi_{\mathfrak{p}}}\left\langle Q_{\mathfrak{p}}, P_{\mathfrak{p}}\right\rangle_{\hat{\phi}_{\mathfrak{p}}}=1
$$

for any $\mathfrak{p} \in \mathbb{S}$. Since a certain power of $w^{2}$ is the identity, it follows that each $w_{T}$ is an automorphism. The automorphism $w_{T}$ extends to the toroidal compactification $\tilde{Z}_{K}$. Via the natural projection $\alpha: \tilde{Z}_{K} \rightarrow \tilde{Y}_{K}$, the automorphisms $w_{T}$ on $\tilde{Z}_{K}$ and $\tilde{Y}_{K}$ are compatible.

\section{Preliminaries on the geometry of $\bar{Y}$}

In this section, we prove some results on the geometry of the special fibre of $Y$, which will be useful in the analytic continuation process of later sections (see Theorem 7.1).

2.1. Directional degrees and valuations. We recall the notions of directional degrees introduced in [29, 43], and directional valuations defined in [15], and 
discuss the relationship between the two notions. Let $Q=(\underline{A}, H)$ be a rigid point of $\tilde{\mathfrak{Y}}_{\text {rig }}$ defined over a finite extension $K^{\prime} / K$.

Case 1. $\underline{A}$ has good reduction over $\mathcal{O}_{K^{\prime}}$. Then $\underline{A}$ and $H$ can be defined over $\mathcal{O}_{K^{\prime}}$. Let $\omega_{H}$ be the module of invariant differential 1-forms of $H$. We have a canonical decomposition $\omega_{H}=\bigoplus_{\beta \in \mathbb{B}} \omega_{H, \beta}$, where each $\omega_{H, \beta}$ is a torsion $\mathcal{O}_{K^{\prime}}$-module generated by one element. So there exists $a_{\beta} \in \mathcal{O}_{K^{\prime}}$ such that $\omega_{H, \beta} \simeq \mathcal{O}_{K^{\prime}} /\left(a_{\beta}\right)$. We set

$$
\operatorname{deg}_{\beta}(Q)=\operatorname{deg}_{\beta}(H)=v_{p}\left(a_{\beta}\right) \in \mathbb{Q} \cap[0,1] .
$$

Case 2. A has semi-stable reduction over $\mathcal{O}_{K^{\prime}}$. Then we have a canonical decomposition $H \simeq \prod_{\mathfrak{p} \in \mathbb{S}} H[\mathfrak{p}]$ of group schemes over $K^{\prime}$. Recall that $A[\mathfrak{p}]$ has a unique maximal $\mathcal{O}_{L^{-}}$-subgroup, denoted by $A[\mathfrak{p}]^{\mu}$, which extends to a group scheme over $\mathcal{O}_{K^{\prime}}$ of multiplicative type. We put, for all $\beta \in \mathbb{B}_{\mathfrak{p}}$,

$$
\operatorname{deg}_{\beta}(Q)= \begin{cases}1 & \text { if } H[\mathfrak{p}]=A[\mathfrak{p}]^{\mu} \\ 0 & \text { otherwise }\end{cases}
$$

We get therefore a parameterization of $\tilde{\mathfrak{Y}}_{\text {rig }}$ by the partial degrees $[15,29,43]$ :

$$
\underline{\operatorname{deg}}=\left(\operatorname{deg}_{\beta}\right)_{\beta \in \mathbb{B}}: \tilde{\mathfrak{Y}}_{\text {rig }} \rightarrow[0,1]^{\mathbb{B}} .
$$

For an ideal $\mathfrak{t}$ of $\mathcal{O}_{L}$ dividing $(p)$, we have $\mathbb{B}=\mathbb{B}_{\mathfrak{t}} \bigsqcup \mathbb{B}_{\mathfrak{t}^{*}}$, and a decomposition of rigid analytic spaces

$$
\tilde{\mathfrak{Y}}_{\text {rig }}-\mathfrak{Y}_{\text {rig }}=\coprod_{(p) \subset \mathfrak{t} \subset \mathcal{O}_{L}}\left(\tilde{\mathfrak{Y}}_{\text {rig }}-\mathfrak{Y}_{\text {rig }}\right)_{\mathfrak{t}},
$$

where $\left(\tilde{\mathfrak{Y}}_{\text {rig }}-\mathfrak{Y}_{\text {rig }}\right)_{\mathfrak{t}}$ consists of points $Q \in \tilde{\mathfrak{Y}}_{\text {rig }}-\mathfrak{Y}_{\text {rig }}$ with $\operatorname{deg}_{\beta}(Q)=1$ for $\beta \in \mathbb{B}_{\mathfrak{t}}$ and $\operatorname{deg}_{\beta}(Q)=0$ for $\beta \in \mathbb{B}_{\mathfrak{t}^{*}}$.

Using the additivity of partial degrees $[43,3.6]$, we have

$$
\operatorname{deg}_{\beta}\left(w_{\mathfrak{p}}(Q)\right)= \begin{cases}1-\operatorname{deg}_{\beta}(Q) & \text { if } \beta \in \mathbb{B}_{\mathfrak{p}} \\ \operatorname{deg}_{\beta}(Q) & \text { if } \beta \notin \mathbb{B}_{\mathfrak{p}}\end{cases}
$$

for any $Q \in \tilde{\mathfrak{Y}}_{\text {rig }}$ and $\mathfrak{p} \in \mathbb{S}$.

Definition 2.2. For $Q \in \tilde{\mathfrak{Y}}_{\text {rig }}$ and $\mathfrak{p} \in \mathbb{S}$, we define $\operatorname{deg}_{\mathfrak{p}}(Q)=\sum_{\beta \in \mathbb{B}_{\mathfrak{p}}} \operatorname{deg}_{\beta}(Q)$. If $\underline{I}=\left\{I_{\mathfrak{p}} \subset\left[0, f_{\mathfrak{p}}\right]: \mathfrak{p} \in \mathbb{S}\right\}$ is a multiset of intervals indexed by $\mathbb{S}$ and $\mathcal{V} \subset \tilde{\mathfrak{Y}}_{\text {rig }}$ is an admissible open subset, we define

$$
\mathcal{V} \underline{I}=\left\{Q \in \mathcal{V}: \operatorname{deg}_{\mathfrak{p}}(Q) \in I_{\mathfrak{p}}, \forall \mathfrak{p} \in \mathbb{S}\right\},
$$


which is an admissible open subset of $\mathcal{V}$. Also, we put $\operatorname{deg}(Q)=\sum_{\mathfrak{p} \in \mathbb{S}} \operatorname{deg}_{\mathfrak{p}}(Q)$ for $Q \in \tilde{\mathfrak{Y}}_{\text {rig }}$, and, for an interval $I \subset[0, g]$, we define

$$
\tilde{\mathfrak{Y}}_{\text {rig }} I=\left\{Q \in \tilde{\mathfrak{Y}}_{\text {rig }}: \operatorname{deg}(Q) \in I\right\} .
$$

We now discuss the relation to partial valuations $v_{\beta}(Q)$ defined in $[15$, Section 4.2], using the mod- $p$ geometry of $\tilde{\mathfrak{Y}}_{\text {rig }}$. The valuations and partial degrees are related as follows:

$$
v_{\beta}(Q)=1-\operatorname{deg}_{\beta}(Q) .
$$

REMARK 2.3. In this paper, we have decided to use the partial degrees which are more intrinsically defined in terms of the subgroup $H$. Since we refer often to the results of [15], and [19], the reader should be mindful of the slight change in the notation. In those references, intervals are formed using directional valuations rather than directional degrees. The two notions are simply related by interchanging $\underline{I}$ with $\underline{I}^{w}$ obtained by replacing any interval $I_{\mathfrak{p}}=[a, b]$, with $I_{\mathfrak{p}}^{w_{\mathfrak{p}}}:=\left[f_{\mathfrak{p}}-b, f_{\mathfrak{p}}-a\right]$ (similarly, for open, half-open intervals).

2.4. Mod- $\boldsymbol{p}$ geometry: stratifications. Recall first Goren and Oort's stratification on $\bar{X}$ defined in [14]. For each $\beta \in \mathbb{B}$, let $h_{\beta} \in \Gamma\left(\bar{X}, \omega_{\sigma^{-1} \circ \beta}^{\otimes p} \otimes \omega_{\beta}^{-1}\right)$ be the $\beta$ th partial Hasse invariant, and let $Z_{\beta} \subseteq \bar{X}$ be the closed subscheme defined by the vanishing of $h_{\beta}$. For any subset $\tau \subseteq \mathbb{B}$, we put $Z_{\tau}=\bigcap_{\beta \in \tau} Z_{\beta}$, and $W_{\tau}=Z_{\tau}-\bigcup_{\tau \subsetneq \tau^{\prime}} Z_{\tau^{\prime}}$. Each $W_{\tau}$ is a locally closed reduced subvariety of $\bar{X}$, equidimensional of codimension $|\tau|$, and $\left\{W_{\tau}, \tau \subseteq \mathbb{B}\right\}$ form a stratification of $\bar{X}$. For any point $\bar{Q} \in \bar{X}$, we put

$$
\tau(\bar{Q})=\left\{\beta \in \mathbb{B}: h_{\beta}(\bar{Q})=0\right\} .
$$

Then, we have $\bar{Q} \in W_{\tau}$ if and only if $\tau(\bar{Q})=\tau$.

Similarly, Goren and Kassaei [15] defined a stratification on $\bar{Y}$. Following loc. cit., we say that a pair $(\varphi, \eta)$ of subsets of $\mathbb{B}$ is admissible if $\ell\left(\varphi^{c}\right) \subset \eta$. For each admissible pair, they defined a locally closed subset $W_{\varphi, \eta}$ equidimensional of codimension $|\varphi|+|\eta|-g$ in $\bar{Y}$. They proved that, if $Z_{\varphi, \eta}$ denotes the closure of $W_{\varphi, \eta}$ in $\bar{Y}$, then

$$
Z_{\varphi, \eta}=\bigcup_{\left(\varphi^{\prime}, \eta^{\prime}\right) \geqslant(\varphi, \eta)} W_{\varphi^{\prime}, \eta^{\prime}},
$$

where $\left(\varphi^{\prime}, \eta^{\prime}\right) \geqslant(\varphi, \eta)$ means that $\varphi^{\prime} \supset \varphi$ and $\eta^{\prime} \supset \eta[15,2.5 .1]$. Then,

$$
\left\{W_{\varphi, \eta}:(\varphi, \eta) \text { admissible }\right\}
$$


form a stratification of $\bar{Y}[15,2.5 .2]$. By [loc. cit. 2.6.4], we have

$$
\pi\left(W_{\varphi, \eta}\right)=\bigcup_{\substack{\varphi \cap \eta \cap \subset \tau \\ \tau \subset(\varphi \cap \eta) \cup\left(\varphi^{c} \cap \eta^{c}\right)}} W_{\tau} .
$$

The relationship between Goren and Kassaei's stratification and the directional degrees can be explained as follows [15, Theorem 4.3.1]. Let sp : $\mathfrak{Y}_{\text {rig }} \rightarrow \bar{Y}$ denote the specialization map. For a rigid point $Q \in \mathfrak{Y}_{\text {rig }}$, we put

$$
\varphi(\bar{Q})=\left\{\beta \in \mathbb{B}, \operatorname{deg}_{\sigma^{-1} \circ \beta}(Q) \in(0,1]\right\}, \eta(\bar{Q})=\left\{\beta \in \mathbb{B}, \operatorname{deg}_{\beta}(Q) \in[0,1)\right\} .
$$

The pair $(\varphi(\bar{Q}), \eta(\bar{Q}))$ is always admissible, and $\bar{Q}=\operatorname{sp}(Q)$ lies in $W_{\varphi, \eta}$ if and only if $(\varphi(\bar{Q}), \eta(\bar{Q}))=(\varphi, \eta)$. Hence, the strata $\left\{W_{\varphi, \eta}\right\}$ correspond bijectively to the faces of the hypercube $[0,1]^{\mathbb{B}}$ : for any stratum $W_{\varphi, \eta}$, we have

$$
\operatorname{sp}^{-1}\left(W_{\varphi, \eta}\right)=\left\{Q \in \mathfrak{Y}_{\text {rig }}:(\varphi(\bar{Q}), \eta(\bar{Q}))=(\varphi, \eta)\right\}=\mathfrak{Y}_{\text {rig }} \mathbf{a},
$$

where $\mathbf{a} \subset[0,1]^{\mathbb{B}}$ is the face consisting of $\left(x_{\beta}\right)_{\beta \in \mathbb{B}} \in[0,1]^{\mathbb{B}}$ with $x_{\beta}=1$ for $\beta \in$ $\eta^{c}, x_{\beta}=0$ for $\beta \in \ell\left(\varphi^{c}\right)$, and $x_{\beta} \in(0,1)$ for $\beta \in \eta \cap \ell(\varphi)$, and $\mathfrak{Y}_{\text {rig }} \mathbf{a}=\operatorname{deg}^{-1}(\mathbf{a})$. Note that the codimension of $W_{\varphi, \eta}$ in $\bar{Y}$ is equal to the dimension of $\mathbf{a}$.

Let $T \subset \mathbb{S}$, and let $w_{T}$ be the Atkin-Lehner automorphism on $\bar{Y}$ defined earlier. The above description of the stratification in terms of directional degrees shows that

$$
w_{T}\left(W_{\varphi, \eta}\right)=W_{w_{T}(\varphi, \eta)},
$$

where $\left(\varphi^{\prime}, \eta^{\prime}\right)=w_{T}(\varphi, \eta)$ is an admissible pair whose $\mathfrak{p}$-component is given by

$$
\left(\varphi_{\mathfrak{p}}^{\prime}, \eta_{\mathfrak{p}}^{\prime}\right)= \begin{cases}\left(r\left(\eta_{\mathfrak{p}}\right), \ell\left(\varphi_{\mathfrak{p}}\right)\right) & \text { if } \mathfrak{p} \in T \\ \left(\varphi_{\mathfrak{p}}, \eta_{\mathfrak{p}}\right) & \text { if } \mathfrak{p} \notin T\end{cases}
$$

DEFINITION 2.5. For a stratum $W_{\varphi, \eta}$ of $\bar{Y}$, the generic part of $W_{\varphi, \eta}$ is defined to be

$$
W_{\varphi, \eta}^{\text {gen }}=\pi^{-1}\left(W_{\varphi \cap \eta}\right) \cap W_{\varphi, \eta} .
$$

LEMMA 2.6. For each admissible pair $(\varphi, \eta), W_{\varphi, \eta}^{\mathrm{gen}}$ is an open dense subset of $W_{\varphi, \eta}$. Moreover, if $\varphi^{c} \cap \eta^{c} \neq \emptyset$, the complement of $W_{\varphi, \eta}^{\mathrm{gen}}$ in $W_{\varphi, \eta}$ is a divisor.

Proof. By (2.4.2), we have $W_{\varphi, \eta}^{\text {gen }}=\left\{\bar{Q} \in W_{\varphi, \eta}: h_{\beta}(\bar{Q}) \neq 0\right.$, for all $\left.\beta \in \varphi^{c} \cap \eta^{c}\right\}$. Therefore, if $\varphi^{c} \cap \eta^{c} \neq \emptyset$, the complement of $W_{\varphi, \eta}^{\text {gen }}$ is the locus where at least one of the sections $\left\{\pi^{*}\left(h_{\beta}\right) \mid \beta \in \varphi^{c} \cap \eta^{c}\right\}$ vanishes. 
Consider now codimension- 0 strata of $\bar{Y}$. They correspond to vertex points of $[0,1]^{\mathbb{B}}$. For a vertex point $\underline{x}=\left(x_{\beta}\right) \in\{0,1\}^{\mathbb{B}}$, we denote by $W_{\underline{x}}=W_{\varphi_{\underline{x}}, \eta_{\underline{x}}}$ the stratum of codimension 0 attached to $\underline{x}$, where

$$
\varphi_{\underline{x}}=\left\{\beta \in \mathbb{B}: \underline{x}_{\sigma^{-1} \circ \beta}=1\right\}, \quad \eta_{\underline{x}}=\left\{\beta \in \mathbb{B}: \underline{x}_{\beta}=0\right\} .
$$

Then, by [15, 4.3.1], we have

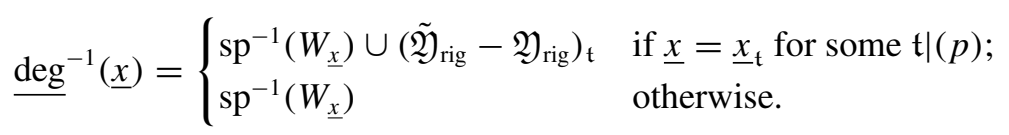

Here, $\underline{x}_{\mathfrak{t}}$ is the vertex point whose $\beta$ th component is 1 if $\beta \in \mathbb{B}_{\mathfrak{t}}$, and 0 otherwise. For a subset $T \subset \mathbb{S}$, let $w_{T}(\underline{x})$ be the vertex point whose $\beta$-component is equal to $1-x_{\beta}$ for $\beta \in \cup_{\mathfrak{p} \in T} \mathbb{B}_{\mathfrak{p}}$, and to $x_{\beta}$ otherwise. It is clear that $w_{T}\left(W_{\underline{x}}\right)=W_{w_{T}(\underline{x})}$.

Definition 2.7. Let $\underline{x} \in[0,1]^{\mathbb{B}}$, and $T \subset \mathbb{S}$. We say that $x$ is $T$-ordinary if, for every prime ideal $\mathfrak{p} \in \bar{T}$, we have either $\underline{x}_{\beta}=1$ for all $\beta \in \mathbb{B}_{\mathfrak{p}}$, or $\underline{x}_{\beta}=0$ for all $\beta \in \mathbb{B}_{\mathfrak{p}}$. If $T=\{\mathfrak{p}\}$ consists of only one element, we also say that $\underline{x}$ is $\mathfrak{p}$-ordinary.

PROPOSITION 2.8. Let $\underline{x}$ be a vertex point of $[0,1]^{\mathbb{B}}$, and $W_{\underline{x}}$ the corresponding stratum of $\bar{Y}$. Let $T$ be the set of all primes $\mathfrak{p} \in \mathbb{S}$ such that $\underline{x}$ is not $\mathfrak{p}$-ordinary. Then, the open dense subset

$$
W_{\underline{x}}^{\mathrm{gen}} \cup w_{T}^{-1}\left(W_{w_{T}(\underline{x})}^{\mathrm{gen}}\right)
$$

of $W_{\underline{x}}$ has a complement of codimension 2.

Proof. Let $W_{\underline{x}}^{\mathrm{sp}}=W_{\underline{x}}-W_{\underline{x}}^{\mathrm{gen}}$. Then, the complement of $W_{\underline{x}}^{\mathrm{gen}} \cup w_{T}^{-1}\left(W_{w_{T}(\underline{x})}^{\mathrm{gen}}\right)$ in $W_{\underline{x}}$ equals

$$
W_{\underline{x}}^{\mathrm{sp}} \cap w_{T}^{-1}\left(W_{w_{T}(\underline{x})}^{\mathrm{sp}}\right) .
$$

By Lemma 2.6, the closed subschemes $W_{\underline{x}}^{\mathrm{sp}}$ and $W_{w_{T}(\underline{x})}^{\mathrm{sp}}$ of $W_{\underline{x}}$ are divisors. To prove the claim, it is enough to show that no irreducible component of $W_{\underline{x}}^{\text {sp }}$ coincides with an irreducible component of $w_{T}^{-1}\left(W_{w_{T}(\underline{x})}^{\mathrm{sp}}\right)$.

Let $Z_{\underline{x}}$ be the closure of $W_{\underline{x}}$ in $\bar{Y}$. In the notation of $[15,2.5 .1]$, we have

$$
Z_{\underline{x}}=Z_{\varphi_{\underline{x}}, \eta_{\underline{x}}}=\bigcup_{(\varphi, \eta) \geqslant\left(\varphi_{\underline{x}}, \eta_{\underline{x}}\right)} W_{\varphi, \eta} .
$$

From the proof of Lemma 2.6, the Zariski closure of $W_{\underline{x}}^{\text {sp }}$ in $Z_{\underline{x}}$ is given by the vanishing of at least one of the sections

$$
\left\{\pi^{*}\left(h_{\beta}\right): \beta \in \varphi_{\underline{x}}^{c} \cap \eta_{\underline{x}}^{c}\right\}
$$


on $Z_{\underline{x}}$. Similarly, using Definition 2.5, we see that the Zariski closure of $w_{T}^{-1}\left(\bar{W}_{w_{T}(\underline{x})}^{\mathrm{sp}}\right)$ in $Z_{\underline{x}}$ is defined by the vanishing of at least one of the sections

$$
\left\{w_{T}^{*} \pi^{*}\left(h_{\beta}\right): \beta \in r_{T}\left(\eta_{\underline{x}}\right)^{c} \cap \ell_{T}\left(\varphi_{\underline{x}}\right)^{c}\right\}
$$

on $Z_{\underline{x}}$.

Lemma 2.9. Let the notation be as in Proposition 2.8. The closure in $Z_{\underline{x}}$ of each irreducible component of $W_{\underline{x}}^{\mathrm{sp}}$ passes through a point in $W_{\mathbb{B}, \mathbb{B}}$.

We assume this Lemma for the moment, and finish the proof of 2.8 as follows. Let $\bar{Q}$ be a point in $W_{\mathbb{B}, \mathbb{B}} \cap Z_{\underline{x}}$ with residue field $\kappa_{\bar{Q}}$. By Stamm's theorem [40], we have an isomorphism

$$
\widehat{\mathcal{O}}_{\bar{Y}, \bar{Q}} \simeq \kappa_{\bar{Q}}\left[\left[x_{\beta}, y_{\beta}: \beta \in \mathbb{B}\right]\right] /\left(x_{\beta} y_{\beta}\right)_{\beta \in \mathbb{B}},
$$

where $x_{\beta}, y_{\beta}$ are specifically defined local parameters. Similarly, we can write

$$
\widehat{\mathcal{O}}_{\bar{Y}, w_{T}(\bar{Q})} \simeq \kappa_{\bar{Q}}\left[\left[x_{\beta}^{\prime}, y_{\beta}^{\prime}: \beta \in \mathbb{B}\right]\right] /\left(x_{\beta}^{\prime} y_{\beta}^{\prime}\right)_{\beta \in \mathbb{B}},
$$

such that $w_{T}^{*}\left(x_{\beta}^{\prime}\right)=y_{\beta}$, and $w_{T}^{*}\left(y_{\beta}^{\prime}\right)=x_{\beta}$ for all $\beta \in \ell_{T}\left(\phi_{\underline{x}}\right)^{c} \cap r_{T}\left(\eta_{\underline{x}}\right)^{c}\left(\subset \cup_{\mathfrak{p} \in T} \mathbb{B}_{\mathfrak{p}}\right)$, as in $[15,2.7 .2]$. It follows from $[15,2.5 .2](4)$ that

$$
\widehat{\mathcal{O}}_{Z_{\underline{x}}, \bar{Q}}=\kappa_{\bar{Q}}\left[\left[z_{\beta}: \beta \in \mathbb{B}\right]\right],
$$

where $z_{\beta}=x_{\beta}$ if $\beta \notin \eta_{\underline{x}}$, and $z_{\beta}=y_{\beta}$ otherwise.

By Lemma 2.9 above, to complete the proof of 2.8, we just need to show that, for each $\beta \in \varphi_{\underline{x}}^{c} \cap \eta_{\underline{x}}^{c}$, and each $\beta^{\prime} \in r_{T}\left(\eta_{\underline{x}}\right)^{c} \cap \ell_{T}\left(\varphi_{\underline{x}}\right)^{c}$, the closed subschemes defined by $\pi^{*}\left(\bar{h}_{\beta}\right)$ and $w_{T}^{*} \pi^{*}\left(h_{\beta^{\prime}}\right)$ in $\operatorname{Spec}\left(\widehat{\mathcal{O}}_{Z_{x}, \bar{Q}}\right)$ have no common irreducible components.

The Key Lemma 2.8.1 in [15] implies that, for all $\beta \in \phi_{\underline{x}}^{c} \cap \eta_{\underline{x}}^{c}$, we have

$$
\pi^{*}\left(h_{\beta}\right)=u x_{\beta}+v y_{\sigma^{-1} \circ \beta}^{p}
$$

in the local ring $\widehat{\mathcal{O}}_{\bar{Y}, \bar{Q}}$, where $u$ and $v$ are units in $\widehat{\mathcal{O}}_{\bar{Y}, \bar{Q}}$. Specializing to the local $\operatorname{ring} \mathcal{O}_{Z_{\underline{x}}, \bar{Q}}$, we obtain

$$
\pi^{*}\left(h_{\beta}\right)=u z_{\beta}+v z_{\sigma^{-1} \circ \beta}^{p} .
$$

Similarly, for all $\beta^{\prime} \in \ell_{T}\left(\phi_{\underline{x}}\right)^{c} \cap r_{T}\left(\eta_{\underline{x}}\right)^{c}$,

$$
\pi^{*}\left(h_{\beta^{\prime}}\right)=u^{\prime} x_{\beta^{\prime}}^{\prime}+v^{\prime}\left(y_{\sigma^{-1} \circ \beta^{\prime}}^{\prime}\right)^{p},
$$

in the local ring $\widehat{\mathcal{O}}_{\bar{Y}, w_{\mathfrak{t}}(\bar{Q})}$, with $u^{\prime}$ and $v^{\prime}$ being units in $\widehat{\mathcal{O}}_{\bar{Y}, w_{\mathfrak{t}}(\bar{Q})}$. This implies that

$$
\begin{aligned}
w_{T}^{*} \pi^{*}\left(h_{\beta^{\prime}}\right) & =w_{T}^{*}\left(u^{\prime}\right) y_{\beta^{\prime}}+w_{T}^{*}\left(v^{\prime}\right)\left(x_{\sigma^{-1} \circ \beta^{\prime}}\right)^{p} \\
& =w_{T}^{*}\left(u^{\prime}\right) z_{\beta^{\prime}}+w_{T}^{*}\left(v^{\prime}\right)\left(z_{\sigma^{-1} \circ \beta^{\prime}}\right)^{p},
\end{aligned}
$$


in the local ring $\mathcal{O}_{Z_{\underline{x}}, \bar{Q}}$. Comparing Equations 2.9.1 and 2.9.3, and noting that

$$
\left(\phi_{\underline{x}}^{c} \cap \eta_{\underline{x}}^{c}\right) \cap\left(\ell_{T}\left(\phi_{\underline{x}}\right)^{c} \cap r_{T}\left(\eta_{\underline{x}}\right)^{c}\right)=\emptyset,
$$

it is now immediate to see that $\pi^{*}\left(h_{\beta}\right)$ and $w_{T}^{*}\left(\pi^{*}\left(h_{\beta^{\prime}}\right)\right)$ cut out two irreducible and distinct divisors in $\operatorname{Spec}\left(\widehat{\mathcal{O}}_{Z_{\underline{x}}, \bar{Q}}\right)$. Now it remains to prove Lemma 2.9.

Proof of Lemma 2.9. Let $C$ be an irreducible component of $W_{\underline{x}}^{\text {sp }}$, and let $\bar{C}$ be its closure in $Z_{\underline{x}}$. Since $Z_{\underline{x}}$ is smooth $[15,2.5 .2].(4)$, there exists a unique irreducible component $\bar{D}$ of $Z_{\underline{x}}$ containing $\bar{C}$. By $[15,2.6 .4](1), \pi(\bar{D})$ is an irreducible component of

$$
\pi\left(Z_{\underline{x}}\right)=\pi\left(Z_{\varphi_{\underline{x}}, \eta_{\underline{x}}}\right)=Z_{\varphi_{\underline{x}} \cap \eta_{\underline{x}}} .
$$

Since $\pi$ is proper, $\pi(\bar{C})$ is an irreducible component of $Z_{\varphi_{\underline{x}} \cap \eta_{\underline{x}}}$. In particular, $\pi(\bar{C})$ intersects $W_{\mathbb{B}}$. On the other hand, since $C \subset \bigcup_{\beta \in \varphi_{\underline{x}}^{c} \cap \eta_{\underline{x}}^{c}} Z_{\underline{x}} \cap \pi^{-1}\left(Z_{\{\beta\}}\right)$, there is $\beta_{0} \in \varphi_{\underline{x}}^{c} \cap \eta_{\underline{x}}^{c}$ such that $\bar{C} \subset \pi^{-1}\left(Z_{\left\{\beta_{0}\right\}}\right)$. Let

$$
E:=\bar{C} \cap Z_{\mathbb{B}-\left\{\beta_{0}\right\}, \mathbb{B}-\left\{\beta_{0}\right\}} .
$$

Since $\bar{C}$ is a divisor in $Z_{\varphi_{\underline{x}} \cap \eta_{\underline{x}}}$, and $Z_{\mathbb{B}-\left\{\beta_{0}\right\}, \mathbb{B}-\left\{\beta_{0}\right\}}$ is purely two-dimensional, every connected component of $\bar{E}$ has dimension one.

By $[15,2.6 .16]$, if $\bar{Q} \in Z_{\mathbb{B}-\left\{\beta_{0}\right\}, \mathbb{B}-\left\{\beta_{0}\right\}}$, then $\tau(\pi(\bar{Q})) \supseteq \mathbb{B}-\left\{\beta_{0}\right\}$. It follows that

$$
\pi(E) \subset W_{\mathbb{B}} \cap \pi(\bar{C}),
$$

which is a finite set, say $\left\{\bar{P}_{1}, \ldots, \bar{P}_{r}\right\}$. Therefore,

$$
E \subset \bigcup_{i=1}^{r} \pi^{-1}\left(\bar{P}_{i}\right) \cap Z_{\mathbb{B}-\left\{\beta_{0}\right\}, \mathbb{B}-\left\{\beta_{0}\right\}} .
$$

By $[15,2.6 .12]$, each $\pi^{-1}\left(\bar{P}_{i}\right) \cap Z_{\mathbb{B}-\left\{\beta_{0}\right\}, \mathbb{B}-\left\{\beta_{0}\right\}}$ is homeomorphic to $\mathbb{A}^{1}$. Since $E$ is purely one-dimensional, it follows that there is a point $\bar{P} \in\left\{\bar{P}_{1}, \ldots, \bar{P}_{r}\right\}$, such that $\pi^{-1}(\bar{P}) \cap Z_{\mathbb{B}-\left\{\beta_{0}\right\}, \mathbb{B}-\left\{\beta_{0}\right\}} \subset E$. Let $\bar{Q}$ be the unique point in

$$
\pi^{-1}(\bar{P})-\pi^{-1}(\bar{P}) \cap Z_{\mathbb{B}-\left\{\beta_{0}\right\}, \mathbb{B}-\left\{\beta_{0}\right\}}=\pi^{-1}(\bar{P}) \cap Z_{\mathbb{B}, \mathbb{B}} .
$$

Since $E$ is Zariski closed in $\bar{Y}$, we deduce that $\bar{Q} \in E \cap W_{\mathbb{B}, \mathbb{B}} \subset \bar{C} \cap W_{\mathbb{B}, \mathbb{B}}$.

\section{Geometric Hilbert modular forms}

For $\underline{k} \in \mathbb{Z}^{\mathbb{B}}$, the space of geometric Hilbert modular forms of level $\Gamma_{1}(N p)$ and weight $\underline{k}$ is defined as

$$
\mathcal{M}_{\underline{k}}\left(\Gamma_{1}(N p) ; K\right):=H^{0}\left(Z_{K}, \omega^{\underline{k}}\right)=H^{0}\left(\tilde{Z}_{K}, \omega^{\underline{k}}\right),
$$


where the last equality is because of the classical Koecher principle [31, 4.9]. We will denote the subspace of cusp forms by $\mathcal{S}_{k}\left(\Gamma_{1}(N p) ; K\right)$. The space of overconvergent Hilbert modular forms of level $\Gamma_{1}(N p)$ and weight $k$ over $K$ is

$$
\mathcal{M}_{\underline{k}}^{\dagger}\left(\Gamma_{1}(N p) ; K\right)=\underset{\mathcal{V} \supset \overrightarrow{\mathcal{Z}}_{\text {rig }}^{\text {ord }}}{\lim } H^{0}\left(\mathcal{V}, \omega^{\underline{k}}\right),
$$

where $\mathcal{V}$ runs through the strict neighborhoods of $\tilde{\mathfrak{Z}}_{\text {rig }}^{\text {ord }}$ in $\tilde{\mathfrak{Z}}_{\text {rig. We will denote the }}$ subspace of cusp forms by $\mathcal{S}_{\underline{k}}^{\dagger}\left(\Gamma_{1}(N p) ; K\right)$.

We have a natural injection $\mathcal{M}_{\underline{k}}\left(\Gamma_{1}(N p) ; K\right) \hookrightarrow \mathcal{M}_{\underline{k}}^{\dagger}\left(\Gamma_{1}(N p) ; K\right)$ sending cusp forms to cusp forms. Both the source and the target of this injection are equipped with an action of Hecke operators, and the injection is compatible with the Hecke action. The overconvergent modular forms in the image of $\mathcal{M}_{\underline{k}}\left(\Gamma_{1}(N p) ; K\right)$ are called classical.

We also define the space of geometric Hilbert modular forms of level $\Gamma_{1}(N) \cap$ $\Gamma_{0}(p)$ and weight $\underline{k}$ as

$$
\mathcal{M}_{\underline{k}}\left(\Gamma_{1}(N) \cap \Gamma_{0}(p) ; K\right):=H^{0}\left(Y_{K}, \omega^{\underline{k}}\right),
$$

with the subspace of cusp forms denoted by $\mathcal{S}_{\underline{k}}\left(\Gamma_{1}(N) \cap \Gamma_{0}(p) ; K\right)$. Similarly, one can define the space of overconvergent Hilbert modular forms of level $\Gamma_{1}(N) \cap$ $\Gamma_{0}(p)$ and weight $\underline{k}$ as

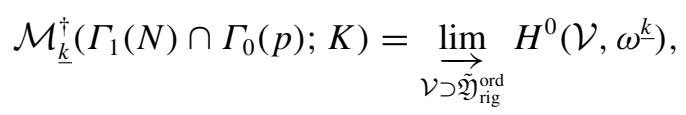

where $\mathcal{V}$ runs through the strict neighborhoods of $\tilde{\mathfrak{Y}}_{\text {rig }}^{\text {ord }}$ in $\tilde{\mathfrak{Y}}_{\text {rig }}$. The subspace of cusp forms is denoted by $\mathcal{S}_{k}^{\dagger}\left(\Gamma_{1}(N) \cap \Gamma_{0}(p) ; K\right)$. When $\underline{k}$ corresponds to parallel weight $k$, we replace $\underline{k}$ with $k$ in the above notation.

REMARK 3.1. Geometric Hilbert modular forms are not exactly automorphic forms for $\operatorname{Res}_{L / \mathbb{Q}} \mathrm{GL}_{2, L}$. In level $\Gamma_{1}(M)$, these automorphic forms can be identified as the space of geometric Hilbert modular forms invariant under the natural action of $\mathcal{O}_{L}{ }^{x,+} / \mathcal{O}_{L, 1, M}^{2}$ (induced by a natural action on $\underline{A}=\left(\underline{A}, \lambda, i_{M}\right)$ via the polarization $\lambda$ ). Here, $\mathcal{O}_{L}{ }^{\times,+}$denotes the group of totally positive units of $L$, and $\mathcal{O}_{L, 1, M}$ the group of units congruent to $1 \bmod M$. See [22, Remark 1.11.8] for more details.

In the second part of this paper, where applications to the Artin conjecture are discussed, we will be working with spaces of automorphic forms for $\operatorname{Res}_{L / \mathbb{Q}} \mathrm{GL}_{2, \mathbb{Q}}$. The results of the first part of the paper will be applied via the above identification. 
3.2. The operators $U_{T}$. Let $T \subset \mathbb{S}$, and set $\mathfrak{t}:=\prod_{\mathfrak{p} \in T} \mathfrak{p}$. We will describe the action of the $U_{T}$-operators. Let $Z_{K}^{T}$ be the étale cover of $Z_{K}$ which classifies triples $(\underline{A}, P, D)$ over $K$-schemes, where $(\underline{A}, P)$ is classified by $Z_{K}$, and $D \subset A[\mathfrak{t}]$ is a closed finite flat $\mathcal{O}_{L}$-subgroup which is an $\mathcal{O}_{L} / \mathfrak{t}$ module, free of rank one as abelian fppf-sheaves, and such that $(P) \cap D=0$. Let $\tilde{Z}_{K}^{T}$ denote the toroidal compactification of $Z_{K}^{T}$, obtained using our fixed choice of collection of cone decompositions. Let $\tilde{\mathfrak{Z}}_{\text {rig }}^{T}$ denote the associated rigid analytic variety. There are two finite flat morphisms

$$
\pi_{1, T}, \pi_{2, T}: \tilde{\mathfrak{Z}}_{\text {rig }}^{T} \rightarrow \tilde{\mathfrak{Z}}_{\text {rig }},
$$

defined by $\pi_{1, T}(\underline{A}, P, D)=(\underline{A}, P)$ and $\pi_{2, T}(\underline{A}, P, D)=(\underline{A} / D, \bar{P})$ on the noncuspidal part, where $\bar{P}$ is the image of $P$ in $A / D$.

We have the same setup over $Y$, that is, for every $T \subset \mathbb{S}$, a rigid analytic variety $\tilde{\mathfrak{Y}}_{\text {rig }}^{T}$ defined over $K$, and two finite flat morphisms

$$
\pi_{1, T}, \pi_{2, T}: \tilde{\mathfrak{Y}}_{\text {rig }}^{T} \rightarrow \tilde{\mathfrak{Y}}_{\text {rig }},
$$

defined similarly. See [19, Section 4] for details. When $T=\{\mathfrak{p}\}$ is a singleton, we write $\tilde{\mathfrak{Z}}_{\text {rig }}^{\mathfrak{p}}$ for $\tilde{\mathfrak{Z}}_{\text {rig }}^{T}$, and $\tilde{\mathfrak{Y}}_{\text {rig }}^{\mathfrak{p}}$ for $\tilde{\mathfrak{Y}}_{\text {rig }}^{T}$, and denote $\pi_{1, T}, \pi_{2, T}$, respectively, with $\pi_{1, \mathfrak{p}}, \pi_{2, \mathfrak{p}}$. If $T=\mathbb{B}$, we use $\tilde{\mathfrak{Z}}_{\text {rig }}^{(p)}$ for $\tilde{\mathfrak{Z}}_{\text {rig }}^{T}$, and $\tilde{\mathfrak{Y}}_{\text {rig }}^{(p)}$ for $\tilde{\mathfrak{Y}}_{\text {rig }}^{T}$, and denote $\pi_{1, T}, \pi_{2, T}$, respectively, with $\pi_{1, p}, \pi_{2, p}$.

Recall the standard construction of Hecke correspondences on the space of overconvergent forms explained in Definition 2.19 of [20]. Let $T \subset \mathbb{S}$, and let $\mathcal{V}_{1}$ and $\mathcal{V}_{2}$ be admissible opens of $\tilde{\mathfrak{Z}}_{\text {rig }}$ such that $\pi_{1, T}^{-1}\left(\mathcal{V}_{1}\right) \subset \pi_{2, T}^{-1}\left(\mathcal{V}_{2}\right)$. We define an operator

$$
U_{T}: \omega^{\underline{k}}\left(\mathcal{V}_{2}\right) \rightarrow \omega^{k}\left(\mathcal{V}_{1}\right)
$$

via the formula

$$
U_{T}(f)=\frac{1}{p^{\sum_{\mathfrak{p} \in T} f_{\mathfrak{p}}}}\left(\pi_{1, T}\right)_{*}\left(\operatorname{res}\left(\operatorname{pr}^{*} \pi_{2, T}^{*}(f)\right)\right),
$$

where res is restriction from $\pi_{2, T}^{-1}\left(\mathcal{V}_{2}\right)$ to $\pi_{1, T}^{-1}\left(\mathcal{V}_{1}\right),\left(\pi_{1, T}\right)_{*}$ is the trace map associated with the finite flat map $\pi_{1, T}$, and $\mathrm{pr}^{*}: \pi_{2, T}^{*} \omega^{\underline{k}} \rightarrow \pi_{1, T}^{*} \omega^{\underline{k}}$ is a morphism of sheaves on $\tilde{\mathfrak{Z}}_{\text {rig }}^{T}$, which at $(\underline{A}, P, D)$ is induced by pr* $: \Omega_{A / D} \rightarrow \Omega_{A}$ coming from the natural projection $\mathrm{pr}: A \rightarrow A / D$. If $f$ is a bounded section of $\underline{\omega}^{k}$ over $\mathcal{V}_{2}$, then $U_{T}(f)$ is also bounded over $\mathcal{V}_{1}$, and we have the estimation

$$
\left|U_{T}(f)\right| \mathcal{V}_{1} \leqslant p^{\sum_{\mathfrak{p} \in T} f_{\mathfrak{p}}}|f|_{\mathcal{V}_{2}} .
$$

When $T=\{\mathfrak{p}\}$ is a singleton, we denote $U_{T}$ by $U_{\mathfrak{p}}$. 
For $0<r<1$, let $\tilde{\mathfrak{Z}}_{\text {rig }}[g-r, g]=\alpha^{-1}\left(\tilde{\mathfrak{Y}}_{\text {rig }}[g-r, g]\right)$ (Definition 2.2). Then, as $r \in \mathbb{Q}$ goes to 0 , the $\tilde{\mathfrak{Z}}_{\text {rig }}[g-r, g]$ form a basis of strict neighborhoods of $\tilde{\mathfrak{Z}}_{\text {rig. }}^{\text {ord }}$ By [19, Section 5], and considering Remark 2.3, for $r$ close to 0, there exists $0<r^{\prime}<r$ such that

$$
\pi_{1, T}^{-1}\left(\tilde{\mathfrak{Z}}_{\text {rig }}[g-r, g]\right) \subset \pi_{2, T}^{-1}\left(\tilde{\mathfrak{Z}}_{\text {rig }}\left[g-r^{\prime}, g\right]\right) .
$$

Taking sections of $\underline{\omega}^{k}$, we get a completely continuous operator $U_{T}$ on $\mathcal{M}_{k}^{\dagger}\left(\Gamma_{1}(N p) ; K\right)$ by formula (3.2.3). It is immediate to see that the operators $U_{T}$ for $T \subset \mathbb{S}$ commute with each other, and $U_{T}$ is the composite of all the $U_{\mathfrak{p}}$ with $\mathfrak{p} \in T$. We put also $U_{p}=U_{\mathbb{S}}$, the composition of all the $U_{\mathfrak{p}}$.

3.3. The operators $w_{T}$. For any $\mathcal{U} \subset \tilde{\mathfrak{Z}}_{\text {rig }}$, and any $T \subset \mathbb{S}$, define

$$
w_{T}: \omega^{\underline{k}}(\mathcal{U}) \rightarrow \omega^{k}\left(w_{T}^{-1}(\mathcal{U})\right),
$$

by $w_{T}(f)=\operatorname{pr}^{*} w_{T}^{*}(f)$. As usual, we set $w_{\mathfrak{p}}:=w_{\{\mathfrak{p}\}}$, and $w=w_{\mathbb{S}}$. The $w_{\mathfrak{p}}$ operators commute with each other, and the operator $w_{T}$ is the composite of all $w_{\mathfrak{p}}$ for $\mathfrak{p} \in T$.

3.4. Nebentypus character. Let $\mathfrak{p} \in \mathbb{S}$, and let $\mathcal{O}_{\mathfrak{p}}$ denote the ring of integers of $L_{\mathfrak{p}}$. For any point $P$ of $A[p]$, we can write $P=P^{\mathfrak{p}} \times P_{\mathfrak{p}}$, where $P^{\mathfrak{p}} \in A[p / \mathfrak{p}]$ and $P_{\mathfrak{p}} \in A[\mathfrak{p}]$. Let $f \in \mathcal{M}_{\underline{k}}^{\dagger}\left(\Gamma_{1}(N p) ; K\right)$. We denote by $\psi_{\mathfrak{p}, f}:\left(\mathcal{O}_{L} / \mathfrak{p}\right)^{\times} \rightarrow \mathbb{C}_{p}^{\times}$ the character given by

$$
f\left(\underline{A}, P^{\mathfrak{p}}, j P_{\mathfrak{p}}\right)=\psi_{\mathfrak{p}, f}(j) f(\underline{A}, P),
$$

for all $j \in\left(\mathcal{O}_{L} / \mathfrak{p}\right)^{\times}$, and we call it the nebentypus character of $f$ at $\mathfrak{p}$. Sometimes, it is convenient to view also $\psi_{\mathfrak{p}, f}$ as a character of $\mathcal{O}_{L_{\mathfrak{p}}}^{\times}$via the natural reduction $\operatorname{map} \mathcal{O}_{L_{\mathfrak{p}}} \rightarrow \mathcal{O}_{L} / \mathfrak{p}$.

\section{Statement of the main result}

In what follows, the Artin reciprocity map is normalized so that any uniformizer is sent to an arithmetic Frobenius. The following is the main classicality result of this work.

THEOREM 4.1. Let $N \geqslant 4$ be an integer not divisible by a prime $p \neq 2$. Consider a collection of elements of $\mathcal{M}_{k}^{\dagger}\left(\Gamma_{1}(N p) ; K\right)$ of parallel weight $k$,

$$
\left\{f_{T}: T \subset \mathbb{S}\right\},
$$


which are all Hecke eigenforms. Let $\psi_{\mathfrak{p}, T}$ denote the nebentypus character of $f_{T}$ at $\mathfrak{p}$. Assume that we are given a collection of Hecke characters of finite order for $L$,

$$
\left\{\chi_{T}: T \subseteq \mathbb{S}\right\} .
$$

Assume that the following conditions hold.

(1) Fix $\mathfrak{p} \in \mathbb{S}$. Then, either for all $\mathfrak{p} \notin T \subset \mathbb{S}$, the conductor of $\chi_{T \cup\{\mathfrak{p}\}} / \chi_{T}$ is $\mathfrak{p n}_{T}$ for some $\mathfrak{n}_{T} \mid N$, or for all $\mathfrak{p} \notin T \subset \mathbb{S}, \chi_{T \cup\{\mathfrak{p}\}} / \chi_{T}=1$. We say that the collection of characters is ramified at $\mathfrak{p}$ in the first case and unramified at $\mathfrak{p}$ in the second case. Furthermore, in all cases, we have

$$
\psi_{\mathfrak{p}, T}^{-1}=\psi_{\mathfrak{p}, T \cup\{\mathfrak{p}\}}=\left.\left(\chi_{T \cup\{\mathfrak{p}\}} / \chi_{T}\right)\right|_{\mathcal{O}_{L_{\mathfrak{p}}}^{\times}},
$$

where in the last equality $\chi_{T \cup\{\mathfrak{p}\}} / \chi_{T}$ is considered as a character of $\mathbb{A}_{L}^{\times} / L^{\times}$ via class field theory.

(2) Each $f_{T}$ is normalized.

(3) If $T \subset \mathbb{S}$, and $\mathfrak{p} \notin T$, then, for any ideal $\mathfrak{m} \subset \mathcal{O}_{L}$ prime to $\mathfrak{p} N$, we have

$$
c\left(f_{T}, \mathfrak{m}\right)=\left(\chi_{T \cup\{\mathfrak{p}\}} / \chi_{T}\right)(\mathfrak{m}) c\left(f_{T \cup\{\mathfrak{p}\}}, \mathfrak{m}\right) .
$$

(4) $c\left(f_{T}, \mathfrak{q}\right)=0$ for all prime ideals $\mathfrak{q} \mid N$.

(5) $c\left(f_{T}, \mathfrak{p}\right) \neq 0$ for all $T \subset \mathbb{S}$.

(6) If $\mathfrak{p} \notin T \subset \mathbb{S}$ and $\chi_{T \cup\{\mathfrak{p}\}} / \chi_{T}=1$, then $c\left(f_{T}, \mathfrak{p}\right) \neq c\left(f_{T \cup\{\mathfrak{p}\}}, \mathfrak{p}\right)$.

Then, all the $f_{T}$ are classical.

The proof of this theorem is divided into three steps. In Section 5, we establish a general analytic continuation result (Theorem 5.8) for an arbitrary finite slope overconvergent form. Then we apply this to each $f_{T}$ as in Theorem 4.1, and show that, after analytic continuation, the various Atkin-Lehren involutions of $f_{T}$ can be glued together to a form defined over a very large area. This will be done in Section 6, in particular Theorem 6.12. Finally, we prove in Theorem 7.1 that the glued form extends automatically to the whole Hilbert modular variety; hence it is classical.

\section{Analytic continuation}

5.1. Admissible domains of $\tilde{\mathfrak{Z}}_{\text {rig. }}$ For a multiset of intervals $\underline{I}$ as in Definition 2.2, set

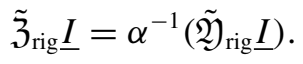

For any admissible open subset $\mathcal{W} \subset \tilde{\mathfrak{Z}}_{\text {rig }}$, define $\mathcal{W} \underline{I}=\mathcal{W} \cap \tilde{\mathfrak{Z}}_{\text {rig }} \underline{I}$. 
5.2. Canonical locus of Goren and Kassaei. For $\mathfrak{p} \in \mathbb{S}$, we put

$$
\mathcal{V}_{\mathfrak{p}}= \begin{cases}\left\{Q \in \tilde{\mathfrak{Y}}_{\text {rig }}: p \operatorname{deg}_{\sigma^{-1} \circ \beta}(Q)+\operatorname{deg}_{\beta}(Q)>1 \text { for } \beta \in \mathbb{B}_{\mathfrak{p}}\right\} & \text { if } f_{\mathfrak{p}}>1 \\ \left\{Q \in \tilde{\mathfrak{Y}}_{\text {rig }}: \operatorname{deg}_{\beta}(Q)>0 \text { for } \beta \in \mathbb{B}_{\mathfrak{p}}\right\} & \text { if } f_{\mathfrak{p}}=1\end{cases}
$$

Following [15], we put

$$
\mathcal{V}_{\text {can }}=\bigcap_{\mathfrak{p} \in \mathbb{S}} \mathcal{V}_{\mathfrak{p}}
$$

This is a slight enlargement of the canonical locus defined by Goren and Kassaei in [15]. Note that the definition here of $\mathcal{V}_{\text {can }}$ is slightly different from that in loc. cit. only at primes $\mathfrak{p}$ with $f_{\mathfrak{p}}=1$.

Lemma 5.3 (Goren and Kassaei). Let $f \in \mathcal{M}_{\underline{k}}^{\dagger}\left(\Gamma_{1}(N p) ; K\right)$ such that $U_{\mathfrak{p}}(f)=$ $a_{\mathfrak{p}} f$ with $a_{\mathfrak{p}} \in \mathbb{C}_{p}^{\times}$for each $\mathfrak{p} \mid p$. Then $f$ extends analytically to a section of $\underline{\omega}^{\underline{k}}$ over $\alpha^{-1}\left(\mathcal{V}_{\text {can }}\right)$.

This lemma is essentially proved in [15]. To extend $f$ along the direction of primes of degree 1, one uses Lubin and Katz's classical theory of canonical subgroups for one-dimensional formal groups. For the extension of $f$ along other directions, one can find a detailed proof for forms of level $\Gamma_{0}(p) \cap \Gamma_{1}(N)$ in [43, Proposition 4.14]. The same arguments work in the $\Gamma_{1}(N p)$-case with $\mathcal{V}_{\text {can }}$ replaced by $\alpha^{-1}\left(\mathcal{V}_{\text {can }}\right)$.

DeFInITION 5.4. Let $W_{\varphi, \eta}$ be a Goren and Kassaei stratum of $\bar{Y}$. For $\mathfrak{p} \in \mathbb{S}$, we say that $W_{\varphi, \eta}$ is not étale at $\mathfrak{p}$ if $\left(\varphi_{\mathfrak{p}}, \eta_{\mathfrak{p}}\right) \neq\left(\emptyset, \mathbb{B}_{\mathfrak{p}}\right)$; equivalently, for every $\bar{Q}=(\underline{A}, H) \in W_{\varphi, \eta}$, the $\mathfrak{p}$-component $H[\mathfrak{p}]$ of the finite group scheme $H$ is not étale. We say that $W_{\varphi, \eta}$ is nowhere étale if $W_{\varphi, \eta}$ is not étale at any $\mathfrak{p} \in \mathbb{S}$.

The nowhere-étale strata have a characterization in terms of partial degrees: a stratum $W_{\varphi, \eta}$ is nowhere étale if and only if we have $\operatorname{deg}_{\mathfrak{p}}(Q)=$ $\sum_{\beta \in \mathbb{B}_{\mathfrak{p}}} \operatorname{deg}_{\beta}(Q)>0$, for every $Q \in \mathrm{sp}^{-1}\left(W_{\varphi, \eta}\right)$ and every $\mathfrak{p} \in \mathbb{S}$.

Definition 5.5. Let $W_{\varphi, \eta}$ be a stratum of codimension 1 . Then, $\ell(\varphi) \cap \eta=\left\{\beta_{0}\right\}$, where $\beta_{0}$ is the unique element of $\mathbb{B}$ such that $\operatorname{deg}_{\beta_{0}}(Q) \in(0,1)$ for one (hence, for all) rigid point $Q \in \operatorname{sp}^{-1}\left(W_{\varphi, \eta}\right)$. We say that $W_{\varphi, \eta}$ is $b a d$ if $\operatorname{deg}_{\sigma \circ \beta_{0}}(Q)=0$ for one (hence, for all) $Q \in \mathrm{sp}^{-1}\left(W_{\varphi, \eta}\right)$; otherwise, we say that $W_{\varphi, \eta}$ is $\operatorname{good}$. In particular, if $\sigma \circ \beta_{0}=\beta_{0}$, that is the prime $\mathfrak{p}_{0} \in \mathbb{S}$ with $\beta \in \mathbb{B}_{\mathfrak{p}_{0}}$ has degree one, then $W_{\varphi, \eta}$ is good. 
DEFINITION 5.6. (i) Let $W=W_{\varphi, \eta}$ be a nowhere-étale stratum of codimension 0 or 1 . We will define an admissible open subset $\left(W^{\text {gen }}\right)^{\prime}$ of $\tilde{\mathfrak{Y}}_{\text {rig }}$ as follows.

(Case 1) $\operatorname{codim}(W)=0$. We put $] W^{\text {gen }}\left[{ }^{\prime}=\operatorname{sp}^{-1}\left(W^{\text {gen }}\right)\right.$, where $W^{\text {gen }}$ is the generic part of $W$ introduced in Definition 2.5.

(Case 2) $\operatorname{codim}(W)=1$. Let $\beta_{0}$ be as in Definition 5.5, $\mathfrak{p}_{0} \in \mathbb{S}$ be such that $\beta_{0} \in \mathbb{B}_{\mathfrak{p}_{0}}$, and $\eta_{\mathfrak{p}_{0}}=\eta \cap \mathbb{B}_{\mathfrak{p}_{0}}$.

(Case 2a) $W$ is good. In this case, we put $] W^{\text {gen }}\left[{ }^{\prime}=\operatorname{sp}^{-1}\left(W^{\text {gen }}\right)\right.$.

(Case 2b) $W$ is bad and $\eta_{\mathfrak{p}_{0}}=\mathbb{B}_{\mathfrak{p}_{0}}$, or, equivalently, for any rigid point $Q$ in $\operatorname{sp}^{-1}(W)$, we have $\operatorname{deg}_{\beta}(Q)=0$ for all $\beta \in \mathbb{B}_{\mathfrak{p}_{0}}-\left\{\beta_{0}\right\}$. In this case, we have $W=W^{\text {gen }}$, and we set

$$
] W^{\text {gen }}\left[^{\prime}=\left\{Q \in \operatorname{sp}^{-1}(W): \operatorname{deg}_{\beta_{0}}(Q) \in\left(\sum_{i=1}^{f_{\mathfrak{p}_{0}}-1} \frac{1}{p^{i}}, 1\right)\right\} .\right.
$$

(Case $2 \mathrm{c}$ ) $W$ is bad and $\eta_{\mathfrak{p}_{0}} \neq \mathbb{B}_{\mathfrak{p}_{0}}$, or, equivalently, there exists an integer $1 \leqslant j \leqslant f_{\mathfrak{p}_{0}}$ such that $\operatorname{deg}_{\sigma^{i} \circ \beta_{0}}(Q)=0$ for $1 \leqslant i \leqslant j$ and $\operatorname{deg}_{\sigma^{j+1} \circ \beta_{0}}(Q)=1$ for any rigid point $Q=(\underline{A}, H) \in \mathrm{sp}^{-1}(W)$. We set $\delta_{j}:=\sum_{i=1}^{j} 1 / p^{i}$, and define

$$
\begin{gathered}
] W^{\text {gen }}\left[^{\prime}=\left\{Q \in \operatorname{sp}^{-1}\left(W^{\mathrm{gen}}\right): \operatorname{deg}_{\beta_{0}}(D) \leqslant \delta_{j},\right.\right. \\
\left.\forall(\underline{A}, H, D) \in \pi_{1, p}^{-1}(Q)\right\},
\end{gathered}
$$

where $\pi_{1, p}: \tilde{\mathfrak{Y}}_{\text {rig }}^{(p)} \rightarrow \tilde{\mathfrak{Y}}_{\text {rig }}$ is defined in (3.2.2).

(ii) We define an admissible domain of $\tilde{\mathfrak{Y}}_{\text {rig }}$ as follows:

$$
\left.\Sigma=\bigcup_{\operatorname{codim}(W)=0,1}\right] W^{\text {gen }}\left[^{\prime},\right.
$$

where $W$ runs though all the nowhere-étale strata of codimension 0 and 1 .

REMARK 5.7. The definition of $] W^{\text {gen }}\left[{ }^{\prime}\right.$ in Case 2(c) will be justified by Proposition 5.15 below.

We can now state the main result of this section.

THEOREM 5.8. Let $f \in \mathcal{M}_{k}^{\dagger}\left(\Gamma_{1}(N p) ; K\right)$ be a simultaneous eigenform for the operators $\left\{U_{\mathfrak{p}}: \mathfrak{p} \in \mathbb{B}\right\}$ with non-zero eigenvalues. Then, $f$ extends analytically to a section of $\underline{\omega}^{\underline{k}}$ over $\alpha^{-1}(\Sigma)$. 
This theorem is an immediate consequence of the following.

Proposition 5.9. Assume that $p \geqslant 3$. Then the image of $\Sigma$ under the Hecke correspondence $U_{p}$ is contained in the canonical locus $\mathcal{V}_{\text {can }}$; that is, we have

$$
\pi_{1, p}^{-1}(\Sigma) \subset \pi_{2, p}^{-1}\left(\mathcal{V}_{\text {can }}\right)
$$

where $\pi_{i, p}: \tilde{\mathfrak{Y}}_{\text {rig }}^{(p)} \rightarrow \tilde{\mathfrak{Y}}_{\text {rig }}$ for $i=1,2$ are the maps defined in (3.2.2).

Indeed, let $f$ be a finite slope eigenform of level $\Gamma_{1}(N p)$ as in Theorem 5.8, and let $\lambda_{p} \neq 0$ be the eigenvalue of $U_{p}$. By Lemma 5.3, $f$ extends to a bounded section of $\underline{\omega}^{k}$ over $\alpha^{-1}\left(\mathcal{V}_{\text {can }}\right)$. Proposition 5.9 implies that $f$ extends to $\alpha^{-1}(\Sigma)$ using the functional equation $f=\frac{1}{\lambda_{p}} U_{p}(f)$. The boundedness of $f$ over $\alpha^{-1}(\Sigma)$ follows from the estimation (3.2.4).

To prove Proposition 5.9, we need some preparation.

5.10. Directional Hodge heights. Let $\underline{A} \in \mathfrak{X}_{\text {rig }}$ be a rigid point, that is, an HBAV defined over the ring of integers $\mathcal{O}_{K^{\prime}}$ of a finite extension $K^{\prime} / K$. We denote by $\underline{A}_{0}$ its base change over $\mathcal{O}_{K^{\prime}} / p$. For each $\beta \in \mathbb{B}$, let $h_{\beta} \in \Gamma\left(\bar{X}, \underline{\omega}_{\sigma-1 \circ \beta}^{p} \otimes \underline{\omega}_{\beta}^{-1}\right)$ be the $\beta$ th partial Hasse invariant, and $h_{\beta}\left(\underline{A}_{0}\right)$ its evaluation at $\underline{A}_{0}$. Using a basis of $\underline{\omega}_{A / \mathcal{O}_{K^{\prime}}}$, we may represent $h_{\beta}\left(\underline{A}_{0}\right)$ as an element of $\mathcal{O}_{K^{\prime}} / p$. In $[43,4.6]$, we defined the $\beta$ th directional Hodge height of $\underline{A}$, denoted by $w_{\beta}(\underline{A})$, as the truncated $p$-adic valuation of $h_{\beta}\left(\underline{A}_{0}\right)$. This is a well-defined rational number in the interval [0,1]. We may extend the definition of $w_{\beta}(\underline{A})$ to whole $\tilde{\mathfrak{X}}_{\text {rig }}$ by setting $w_{\beta}(\underline{A})=0$ if $\underline{A}$ is a rigid point in $\tilde{\mathfrak{X}}_{\text {rig }}-\mathfrak{X}_{\text {rig. }}$. In $[15,4.2]$, the same notion is defined under the name of valuations on $\mathfrak{X}_{\text {rig }}$, and the notation $v_{\beta}$ is used instead of $w_{\beta}$. The following Lemma will play a crucial role in the proof of Proposition 5.9.

LEMMA 5.11 (Goren and Kassaei). Let $Q=(\underline{A}, H) \in \tilde{\mathfrak{Y}}_{\text {rig }}$ be a rigid point. Then, for each $\beta \in \mathbb{B}$, we have

$$
w_{\beta}(\underline{A}) \geqslant \min \left\{p \operatorname{deg}_{\sigma^{-1} \circ \beta}(H), 1-\operatorname{deg}_{\beta}(H)\right\} .
$$

Moreover, the equality holds if $p \operatorname{deg}_{\sigma^{-1} \circ \beta}(H) \neq 1-\operatorname{deg}_{\beta}(H)$.

Proof. Note that, if $\beta \notin \varphi(\bar{Q}) \cap \eta(\bar{Q})$, then the statement is obvious using (2.4.3). For the remaining cases, the statement follows directly from Goren and Kassaei's 'Key Lemma' in [15]. For another proof, see also [43, 4.5].

Proof of Proposition 5.9. Let $\pi_{1, p}, \pi_{2, p}: \tilde{\mathfrak{Y}}_{\text {rig }}^{(p)} \rightarrow \tilde{\mathfrak{Y}}_{\text {rig }}$ be as in (3.2.2). To prove Proposition 5.9, it suffices to show that, for every rigid point $Q=(\underline{A}, H)$ of 
$\Sigma \subset \tilde{\mathfrak{Y}}_{\text {rig }}$, we have $\pi_{2, p}\left(\pi_{1, p}^{-1}\right)(Q) \subset \mathcal{V}_{\text {can }}$. Note that

$\pi_{1, p}^{-1}(Q)=\left\{(\underline{A}, H, D): D \subset A[p]\right.$ is free of rank one over $\mathcal{O}_{L} / p$ and $\left.D \cap H \neq 0\right\}$.

By the definition of $\mathcal{V}_{\text {can }}$, we have to show that, if $(\underline{A}, H, D) \in \pi_{1, p}^{-1}(Q)$, and $\beta \in$ $\mathbb{B}_{\mathfrak{p}} \subset \mathbb{B}$ such that $f_{\mathfrak{p}} \neq 1$, then we have $p \operatorname{deg}_{\beta}(\underline{A}[p] / D)+\operatorname{deg}_{\sigma \circ \beta}(\underline{A}[p] / D)>1$; equivalently, for all $(\underline{A}, H, D)$ and $\beta$ as above, we must show that

$$
p \operatorname{deg}_{\beta}(D)+\operatorname{deg}_{\sigma \circ \beta}(D)<p,
$$

since $\operatorname{deg}_{\beta}(\underline{A}[p] / D)=1-\operatorname{deg}_{\beta}(D)$ for $\beta \in \mathbb{B}$.

LEMMA 5.12. Let $Q=(\underline{A}, H)$ be a rigid point of $\tilde{\mathfrak{Y}}_{\text {rig }}$ whose specialization $\bar{Q}$ is generic in the sense of Definition 2.5. Let $(\underline{A}, H, D) \in \pi_{1, p}^{-1}(Q), \mathfrak{p} \in \mathbb{S}$, and $\beta \in \mathbb{B}_{\mathfrak{p}}$.

(1) We have

$$
\operatorname{deg}_{\beta}(H)+\operatorname{deg}_{\beta}(D) \leqslant \sum_{i=0}^{f_{\mathfrak{p}}-1} \frac{1}{p^{i}} .
$$

In particular, we have $\operatorname{deg}_{\beta}(D)<1$ if $\operatorname{deg}_{\beta}(H)>\sum_{i=1}^{f_{\mathfrak{p}}-1} \frac{1}{p^{i}}$, and $\operatorname{deg}_{\beta}(D) \leqslant$ $\sum_{i=1}^{f_{\mathfrak{p}}-1} \frac{1}{p^{i}}$, if $\operatorname{deg}_{\beta}(H)=1$.

(2) If $\operatorname{deg}_{\beta}(H)=1$, then $\operatorname{deg}_{\sigma^{-1} \circ \beta}(D)=0$.

(3) If $\operatorname{deg}_{\sigma^{-1} \circ \beta}(H)=0$ and $\operatorname{deg}_{\beta}(D)<1$, then $\operatorname{deg}_{\sigma^{-1} \circ \beta}(D)=0$.

Proof. The second part of (1) is clearly a consequence of the first part. Suppose that $(\underline{A}, H, D)$ are defined over $\mathcal{O}_{K^{\prime}}$, where $K^{\prime} / K$ is a finite extension. Consider the homomorphism $D[\mathfrak{p}] \rightarrow A[\mathfrak{p}] / H[\mathfrak{p}]$ of finite flat group schemes of onedimensional $\mathbb{F}_{p^{f \mathfrak{p}}}$-vector spaces, which is generically an isomorphism. By Raynaud's theory, one gets $[43,3.7]$

$$
\sum_{i=0}^{f_{\mathfrak{p}}-1} p^{f_{\mathfrak{p}}-1-i} \operatorname{deg}_{\sigma^{i} \circ \beta}(D) \leqslant \sum_{i=0}^{f_{\mathfrak{p}}-1} p^{f_{\mathfrak{p}}-1-i} \operatorname{deg}_{\sigma^{i} \circ \beta}(A / H) .
$$

Statement (i) follows from the fact that $\operatorname{deg}_{\gamma}(A / H)=1-\operatorname{deg}_{\gamma}(H)$ for all $\gamma \in \mathbb{B}$.

For statement (2), the assumption on $\operatorname{deg}_{\beta}(H)$ implies that $\beta \notin \eta(\bar{Q})$ (by (2.4.3)), whence $\beta \notin \varphi(\bar{Q}) \cap \eta(\bar{Q})$. Since the specialization $\bar{Q}$ of $Q$ is generic, it follows that $w_{\beta}(A)=0$. Applying Lemma 5.11 to $(\underline{A}, D)$, we get $\operatorname{deg}_{\sigma^{-1} \circ \beta}(D)=0$ in view of $\operatorname{deg}_{\beta}(D)<1$. 
For (3), the assumption on $\operatorname{deg}_{\sigma^{-1} \circ \beta}(H)$ implies that $\beta \notin \varphi(\bar{Q})$. The rest of the argument is the same as in part (2).

We assume that $Q=(\underline{A}, H) \in] W^{\text {gen }}\left[{ }^{\prime} \subseteq \Sigma\right.$, where $W=W_{\varphi, \eta}$ is a nowhereétale stratum of codimension 0 or 1 . Let $(\underline{A}, H, D) \in \pi_{1, p}^{-1}(Q)$ be a rigid point of $\tilde{\mathfrak{Y}}_{\text {rig }}^{(p)}$ above $Q$.

LEMMA 5.13. Using the above notation, let $\mathfrak{p} \in \mathbb{S}$ be such that $\operatorname{deg}_{\beta}(H) \in$ $\{0,1\}$, for all $\beta \in \mathbb{B}_{\mathfrak{p}}$. Then, we have $\operatorname{deg}_{\beta}(D)=0$ for all $\beta \in \mathbb{B}_{\mathfrak{p}}$ unless $\operatorname{deg}_{\beta}(H)=1$ and $\operatorname{deg}_{\sigma \circ \beta}(H)=0$, in which case we have

$$
\frac{1}{p} \leqslant \operatorname{deg}_{\beta}(D) \leqslant \sum_{i=1}^{f_{\mathfrak{p}}-1} \frac{1}{p^{i}} .
$$

In particular, (5.11.1) holds for every $\beta \in \mathbb{B}_{\mathfrak{p}}$.

Proof. By Lemma 5.12(2), if $\operatorname{deg}_{\sigma \circ \beta}(H)=1$, then $\operatorname{deg}_{\beta}(D)=0$. We now prove that $\operatorname{deg}_{\beta}(D)=0$ if $\operatorname{deg}_{\beta}(H)=0$. The assumption that $W_{\varphi, \eta}$ is nowhere étale implies that $\operatorname{deg}_{\beta}(H)$ cannot be 0 for all $\beta \in \mathbb{B}_{\mathfrak{p}}$. There exists an integer $n \geqslant 1$ such that $\operatorname{deg}_{\sigma^{i} \circ \beta}(H)=0$ for $0 \leqslant i \leqslant n-1$ and $\operatorname{deg}_{\sigma^{n} \circ \beta}(H)=1$. Lemma 5.12(2) implies that $\operatorname{deg}_{\sigma^{n-1} \circ \beta}(D)=0$. If $n=1$, then we are done; if $n \geqslant 2$, then it follows from repeated application of Lemma 5.12(3) that $\operatorname{deg}_{\sigma^{i} \circ \beta}(D)=0$ for $0 \leqslant i \leqslant n-2$.

It follows from the above that $\operatorname{deg}_{\beta}(D)=0$ except if $\operatorname{deg}_{\beta}(H)=1$ and $\operatorname{deg}_{\sigma \circ \beta}(H)=0$. Then, $\operatorname{deg}_{\beta}(D) \leqslant \sum_{i=1}^{f_{\mathfrak{p}}} 1 / p^{i}$ follows from Lemma 5.12(1). It remains to show that $\operatorname{deg}_{\beta}(D) \geqslant 1 / p$. By Lemma 5.11, we get $w_{\sigma \circ \beta}(A)=1$. Applying the same lemma to $(\underline{A}, D)$, we obtain

$$
1=w_{\sigma \circ \beta}(A) \geqslant \min \left\{1-\operatorname{deg}_{\sigma \circ \beta}(D), p \operatorname{deg}_{\beta}(D)\right\} .
$$

Since $\operatorname{deg}_{\sigma \circ \beta}(D)=0$ by the first part of the proof, it follows that $\operatorname{deg}_{\beta}(D) \geqslant$ $1 / p$.

We now come back to the proof of Proposition 5.9. By the discussion above, it suffices to check (5.11.1) for all $\beta \in \mathbb{B}$. Assume first that $\operatorname{codim}(W)=0$. In this case, Lemma 5.13 applies to every prime $\mathfrak{p} \in \mathbb{S}$. Hence, (5.11.1) holds for every $\beta \in \mathbb{B}$.

Assume now that $\operatorname{codim}(W)=1$. Let be the unique element of $\mathbb{B}$ with $\operatorname{deg}_{\beta_{0}}(H) \in(0,1)$, and let $\mathfrak{p}_{0} \in \mathbb{S}$ be such that $\beta_{0} \in \mathbb{B}_{\mathfrak{p}_{0}}$. Let $\mathfrak{p} \in \mathbb{S}$. If $\mathfrak{p} \neq \mathfrak{p}_{0}$, then $\operatorname{deg}_{\beta}(H) \in\{0,1\}$ for $\beta \in \mathbb{B}_{\mathfrak{p}}$. By Lemma 5.13, relation (5.11.1) holds for $\beta \in \mathbb{B}_{\mathfrak{p}}$. Consider now the case $\mathfrak{p}=\mathfrak{p}_{0}$. We can assume that $f_{\mathfrak{p}_{0}}>1$. We distinguish two cases. 
(a) Assume that $\operatorname{deg}_{\sigma^{-1} \circ \beta_{0}}(H)=\operatorname{deg}_{\sigma \circ \beta_{0}}(H)=1$. We deduce first from Lemma 5.12 (respectively, parts (1) and (2)) that $\operatorname{deg}_{\sigma^{-1} \circ \beta}(D)<1$, and $\operatorname{deg}_{\beta_{0}}(D)=0$. This implies that relation (5.11.1) is satisfied for $\beta=\sigma^{-1} \circ \beta_{0}, \beta_{0}$. For the rest of the $\beta$ the result follows from an argument exactly as in the proof of Lemma 5.13.

(b) Assume that $\operatorname{deg}_{\sigma^{-1} \circ \beta_{0}}(H)=0$ and $\operatorname{deg}_{\sigma \circ \beta_{0}}(H)=1$. Lemma 5.12(2) implies that $\operatorname{deg}_{\beta_{0}}(D)=0$. Applying Lemma 5.12(3), we further find that $\operatorname{deg}_{\sigma^{-1} \circ \beta_{0}}(D)=$ 0 . This gives relation (5.11.1) for $\beta=\sigma^{-1} \circ \beta_{0}, \beta_{0}$. For the rest of the $\beta$, we could use an argument similar to the proof of Lemma 5.13, if we prove that $\operatorname{deg}_{\beta}(D)=0$ if $\operatorname{deg}_{\beta}(H)=0$. One can find an $\ell \geqslant 1$ such that $\operatorname{deg}_{\sigma^{i} \circ \beta}(H)=0$ for $0 \leqslant i \leqslant \ell-1$, and $\operatorname{deg}_{\sigma_{\circ}{ }_{\circ}}(H) \neq 0$. If the latter value is 1 , the proof of loc. cit works. If not, then $\sigma^{\ell} \circ \beta=\beta_{0}$, whence $\operatorname{deg}_{\sigma^{\ell} \circ \beta}(D)=0 \neq 1$. Successive application of Lemma 5.12(3) gives the desired result.

Now, we assume that $W$ is bad; that is, $\operatorname{deg}_{\sigma \circ \beta_{0}}(H)=0$. Note that in this case, for any $D$ as above, we have $\operatorname{deg}_{\beta_{0}}(D)<1$ (by Lemma 5.12(1) in case 2b, and by definition in case $2 \mathrm{c}$ ). We have the following two cases.

(c) $\operatorname{deg}_{\sigma^{-1} \circ \beta_{0}}(H)=1$. First, the same arguments as in the proof of Lemma 5.13 show that $\operatorname{deg}_{\beta}(D)=0$ whenever $\operatorname{deg}_{\beta}(H)=0$, and one verifies easily (5.11.1) for $\beta \neq \sigma^{-2} \circ \beta_{0}, \sigma^{-1} \circ \beta_{0}, \beta_{0}$. By Lemma 5.12(2), we have $\operatorname{deg}_{\sigma^{-2} \circ \beta_{0}}(D)=0$, which implies (5.11.1) for $\beta=\sigma^{-2} \circ \beta_{0}$. One verifies (5.11.1) for $\beta=\beta_{0}$, since $\operatorname{deg}_{\sigma \circ \beta_{0}}(D)=0$ (owing to $\operatorname{deg}_{\sigma \circ \beta_{0}}(H)=0$ ), and $\operatorname{deg}_{\beta_{0}}(D)<1$ (by definition of $\left.\left(W^{\text {gen }}\right)^{\prime}\right)$. It remains to deal with $\beta=\sigma^{-1} \circ \beta_{0}$. Using Lemma 5.11, we have

$$
w_{\beta_{0}}(A)=1-\operatorname{deg}_{\beta_{0}}(H) \geqslant \min \left\{p \operatorname{deg}_{\sigma^{-1} \circ \beta_{0}}(D), 1-\operatorname{deg}_{\beta_{0}}(D)\right\} .
$$

Relation (5.11.1) is trivially true if $p \operatorname{deg}_{\sigma^{-1} \circ \beta_{0}}(D) \leqslant 1-\operatorname{deg}_{\beta_{0}}(D)$. Assume then that $p \operatorname{deg}_{\sigma^{-1} \circ \beta_{0}}(D)>1-\operatorname{deg}_{\beta_{0}}(D)$. In this case, we have $\operatorname{deg}_{\beta_{0}}(D)=$ $1-w_{\beta_{0}}(A)=\operatorname{deg}_{\beta_{0}}(H)$. By Lemma 5.12(i), we have $\operatorname{deg}_{\beta_{0}}(D)=\operatorname{deg}_{\beta_{0}}(H) \leqslant$ $(1 / 2) \sum_{i=0}^{f_{\mathfrak{p}_{0}}-1} 1 / p^{i}$, and $\operatorname{deg}_{\sigma^{-1} \circ \beta_{0}}(D) \leqslant \sum_{i=1}^{f_{\mathfrak{p}_{0}}-1} 1 / p^{i}$. Hence, we get

$$
p \operatorname{deg}_{\sigma^{-1} \circ \beta_{0}}(D)+\operatorname{deg}_{\beta_{0}}(D)<\frac{3}{2} \sum_{i=0}^{f_{\mathfrak{p}_{0}}-1} 1 / p^{i}<\frac{3 p}{2(p-1)}<p .
$$

Note that we used $p \geqslant 3$ in the last step. This verifies (5.11.1) for $\beta=\sigma^{-1} \circ \beta_{0}$.

(d) $\operatorname{deg}_{\sigma^{-1} \circ \beta_{0}}(H)=0$. Since $\operatorname{deg}_{\beta_{0}}(D)<1$, the proof of Lemma 5.13, as modified in part (b) above, allows us to verify (5.11.1) for $\beta \neq \sigma^{-1} \circ \beta_{0}, \beta_{0}$. In the course of doing so, we prove that $\operatorname{deg}_{\beta}(D)=0$ whenever $\operatorname{deg}_{\beta}(H)=0$. In particular, we have $\operatorname{deg}_{\sigma^{-1} \circ \beta_{0}}(D)=\operatorname{deg}_{\sigma \circ \beta_{0}}(D)=0$. Since $\operatorname{deg}_{\beta_{0}}(D)<1$, we conclude that (5.11.1) holds for $\beta=\sigma^{-1} \circ \beta_{0}, \beta_{0}$ as well.

The proof of Proposition 5.9 is now complete. 
The following lemma will be used to prove certain connectedness results in the next section. It also justifies the definition of $\left(W^{\text {gen }}\right)^{\prime}$ in Case $2 c$ of Definition 5.6.

LEMMA 5.14. Let $W$ be a bad stratum of codimension 1 as in Case 2(c) of Definition 5.6, $\beta_{0} \in \mathbb{B}, j \in \mathbb{Z}_{\geqslant 1}$, and let $\delta_{j}$ be as defined there. Let $Q=$ $(\underline{A}, H) \in \mathrm{sp}^{-1}\left(W^{\mathrm{gen}}\right)$ be a rigid point, $(\underline{A}, H, D) \in \pi_{1, p}^{-1}(Q)$. Then, we have

(1) $\operatorname{deg}_{\beta_{0}}(D)=\delta_{j}$, if $\operatorname{deg}_{\beta_{0}}(H)>\delta_{j}$;

(2) $\operatorname{deg}_{\beta_{0}}(D)=\operatorname{deg}_{\beta_{0}}(H)$, if $\operatorname{deg}_{\beta_{0}}(H)<\delta_{j}$;

(3) $\operatorname{deg}_{\beta_{0}}(D) \geqslant \delta_{j}$, if $\operatorname{deg}_{\beta_{0}}(H)=\delta_{j}$.

Proof. We will prove this lemma using Breuil-Kisin modules. Let $K^{\prime} / K$ be a finite extension with ramification index $e$ and residue field $\kappa^{\prime}$ such that $(A, H)$ and $D$ can be defined over $\mathcal{O}_{K^{\prime}}$. Let $\mathfrak{S}_{1}=\kappa^{\prime}[[u]]$, and let $\varphi: \mathfrak{S}_{1} \rightarrow \mathfrak{S}_{1}$ be the Frobenius endomorphism.

Let $\mathfrak{p}_{0} \in \mathbb{S}$ with $\beta_{0} \in \mathbb{B}_{\mathfrak{p}_{0}}$. Since the lemma concerns only the $p$-divisible group $A\left[\mathfrak{p}_{0}^{\infty}\right]$, to simplify the notation, we may assume that $p$ is inert in $F$. Let $(\mathfrak{M}, \varphi)$ be the contravariant Breuil-Kisin module of $A[p]$ as considered in [43, Section 3]. Recall that the action of $\mathcal{O}_{L}$ on $A[p]$ induces a canonical decomposition $\mathfrak{M}=$ $\bigoplus_{\beta \in \mathbb{B}} \mathfrak{M}_{\beta}$, where $\mathfrak{M}_{\beta}$ is a free $\mathfrak{S}_{1}$-module of rank two on which $\mathcal{O}_{L}$ acts via $\beta: \mathcal{O}_{L} \rightarrow W(\kappa) \rightarrow \kappa^{\prime}$. The Frobenius semi-linear endomorphism $\varphi$ on $\mathfrak{M}$ sends $\mathfrak{M}_{\sigma^{-1} \circ \beta}$ to $\mathfrak{M}_{\beta}$ for $\beta \in \mathbb{B}$. By the main theorem of Kisin modules, the $\mathcal{O}_{L^{-}}$ equivariant quotient $A[p] \rightarrow A[p] / H$ corresponds to an $\mathcal{O}_{L}$-equivariant Kisin submodule $\mathfrak{L}=\bigoplus_{\beta \in \mathbb{B}} \mathfrak{L}_{\beta} \subseteq \mathfrak{M}$ such that, for each $\beta \in \mathbb{B}$, both $\mathfrak{L}_{\beta}$ and $\mathfrak{M}_{\beta} / \mathfrak{L}_{\beta}$ are both free $\mathfrak{S}_{1}$-module of rank one. For each $\beta$, we choose a basis $\left(\mathbf{e}_{\beta}, \mathbf{f}_{\beta}\right)$ for $\mathfrak{M}_{\beta}$ over $\mathfrak{S}_{1}$ such that $\mathfrak{L}_{\beta}$ is generated by $\mathbf{e}_{\beta}$. Then the endomorphism $\varphi$ is given by

$$
\varphi\left(\mathbf{e}_{\sigma^{-1} \circ \beta}, \mathbf{f}_{\sigma^{-1} \circ \beta}\right)=\left(\mathbf{e}_{\beta}, \mathbf{f}_{\beta}\right)\left(\begin{array}{cc}
a_{\beta} & b_{\beta} \\
0 & d_{\beta}
\end{array}\right),
$$

with $a_{\beta}, b_{\beta}, d_{\beta} \in \mathfrak{S}_{1}$. By abuse of notation, we denote by $v_{p}$ the valuation on $\mathfrak{S}_{1}$ normalized by $v_{p}(u)=1 / e$. We list the properties of $a_{\beta}, b_{\beta}, d_{\beta}$ as follows.

- By [43, Lemma 3.9], we have $v_{p}\left(d_{\beta}\right)=\operatorname{deg}_{\beta}(H)$, and $v_{p}\left(a_{\beta}\right)=\operatorname{deg}_{\beta}(A[p] / H)$ $=1-\operatorname{deg}_{\beta}(H)$.

- If $\operatorname{deg}_{\beta}(H)=0$, that is $d_{\beta} \in \mathfrak{S}_{1}^{\times}$, then we may assume that $b_{\beta}=0$ and $d_{\beta}=1$ up to replacing $\mathbf{f}_{\beta}$ by $\varphi\left(\mathbf{f}_{\sigma^{-1} \circ \beta}\right)$.

- If $Q \in \mathrm{sp}^{-1}\left(W^{\text {gen }}\right)$, then $v_{p}\left(b_{\sigma^{j+1} \circ \beta_{0}}\right)=0$ by [43, Lemma 3.12]. 
- The fact that $\operatorname{deg}_{\beta_{0}}(H) \in(0,1)$ implies that $v_{p}\left(b_{\beta_{0}}\right)=0$, since the cokernel of the linearized map $1 \otimes \varphi: \varphi^{*}\left(\mathfrak{M}_{\sigma^{-1} \circ \beta}\right) \rightarrow \mathfrak{M}_{\beta}$ is free of rank one over $\mathfrak{S}_{1} / u^{e}$ (see $[43,3.10])$.

Let $D \subseteq A[p]$ be an $\mathcal{O}_{F}$-stable subgroup scheme such that $(\underline{A}, H, D) \in \pi_{1, p}^{-1}$ $(\underline{A}, H)$, and let $\mathfrak{N}=\bigoplus_{\beta \in \mathbb{B}} \mathfrak{N}_{\beta} \subseteq \mathfrak{M}$ be the Kisin submodule corresponding to $A[p] / D$. Assume that $\mathfrak{N}_{\sigma^{-1} \circ \beta_{0}}=\mathfrak{S}_{1}\left(\mathbf{e}_{\sigma^{-1} \circ \beta_{0}}+y_{\sigma^{-1} \circ \beta_{0}} \mathbf{f}_{\sigma^{-1} \circ \beta_{0}}\right)$. Since $\mathfrak{N}_{\sigma^{-1} \circ \beta_{0}}$ is stable under $\varphi^{g}$, the variable $y_{\sigma^{-1} \circ \beta_{0}}$ has to satisfy the following equation:

$$
\begin{gathered}
a_{\sigma^{-1} \circ \beta_{0}} \cdots a_{\sigma^{-g+1} \circ \beta_{0}}^{p^{g-2}} a_{\beta_{0}}^{p^{g-1}}+\left(\sum_{i=0}^{g-1} a_{\sigma^{-1} \circ \beta_{0}} \cdots a_{\sigma^{-i} \circ \beta_{0}}^{p^{i-1}} b_{\sigma^{-i-1} \circ \beta_{0}}^{p^{i}} d_{\sigma^{-i-2} \circ \beta_{0}}^{p^{i+1}} \cdots d_{\sigma^{-g} \circ \beta_{0}}^{p^{g-1}}\right) \\
\times y_{\sigma^{-1} \circ \beta_{0}}^{p^{g}}=d_{\sigma^{-1} \circ \beta_{0}} \cdots d_{\sigma^{-g+1} \circ \beta_{0}}^{p^{g-2}} d_{\beta_{0}}^{g-1} y_{\sigma^{-1} \circ \beta_{0}}^{p^{g}-1} .
\end{gathered}
$$

Note that the constant term has valuation

$$
v_{p}\left(a_{\sigma^{-1} \circ \beta_{0}} \cdots a_{\sigma^{-g+1} \circ \beta_{0}}^{p^{g-2}} a_{\beta_{0}}^{p^{g-1}}\right)=\sum_{i=1}^{g} p^{i-1}\left(1-\operatorname{deg}_{\sigma^{-i} \circ \beta_{0}}(H)\right),
$$

and the coefficient of $y_{\sigma^{-1} \circ \beta_{0}}^{p^{g}-1}$ has valuation

$$
v_{p}\left(d_{\sigma^{-1}} \cdots d_{\sigma^{-g+1} \circ \beta_{0}}^{p^{g-2}} d_{\beta_{0}}^{g-1}\right)=\sum_{i=1}^{g} p^{i-1} \operatorname{deg}_{\sigma^{-i} \circ \beta_{0}}(H) .
$$

Let $c_{p^{g}}=\sum_{i=0}^{g-1} a_{\sigma^{-1} \circ \beta_{0}} \cdots a_{\sigma^{-i} \circ \beta_{0}}^{p^{i-1}} b_{\sigma^{-i-1} \circ \beta_{0}}^{p^{i}} d_{\sigma^{-i-2} \circ \beta_{0}}^{p^{i+1}} \cdots d_{\sigma^{g} \circ \beta_{0}}^{p^{g-1}}$ denote the coefficient of $y_{\sigma^{-1} \circ \beta_{0}}^{p^{g}}$, and let $j$ and $\delta_{j}$ be as defined in the lemma. We distinguish three cases.

(1) If $\operatorname{deg}_{\beta_{0}}(H)>\delta_{j}$, we have

$$
\begin{aligned}
v_{p}\left(c_{p^{g}}\right) & =v_{p}\left(a_{\sigma^{-1} \circ \beta_{0}} \cdots a_{\sigma^{-g+1} \circ \beta_{0}}^{p^{g-2}} b_{\beta_{0}}^{p^{g-1}}\right) \\
& =\sum_{i=1}^{g-1} p^{i-1}\left(1-\operatorname{deg}_{\sigma^{-i} \circ \beta_{0}}(H)\right) .
\end{aligned}
$$

By the method of the Newton polygon, all the $p^{g}$-solutions of $y_{\sigma^{-1} \circ \beta_{0}}$ have valuation

$$
v_{p}\left(y_{\sigma^{-1} \circ \beta_{0}}\right)=\frac{1}{p}\left(1-\operatorname{deg}_{\beta_{0}}(H)\right) .
$$

Since $\varphi\left(\mathbf{e}_{\sigma^{-1} \circ \beta_{0}}+y_{\sigma^{-1} \circ \beta_{0}} \mathbf{f}_{\sigma^{-1} \circ \beta_{0}}\right)=\left(a_{\beta_{0}}+b_{\beta_{0}} y_{\sigma^{-1} \circ \beta_{0}}^{p}\right) \mathbf{e}_{\beta_{0}}+y_{\sigma^{-1} \circ \beta_{0}}^{p} d_{\beta_{0}} \mathbf{f}_{\beta_{0}}$, a detailed computation shows that

$$
\begin{aligned}
\min \left\{v_{p}\left(a_{\beta_{0}}+b_{\beta_{0}} y_{\sigma^{-1} \circ \beta_{0}}^{p}\right), v_{p}\left(y_{\sigma^{-1} \circ \beta_{0}}^{p} d_{\beta_{0}}\right)\right\} & =v_{p}\left(a_{\beta_{0}}+b_{\beta_{0}} y_{\sigma^{-1} \circ \beta_{0}}^{p}\right) \\
& =1-\delta_{j} .
\end{aligned}
$$


Since $\mathfrak{N}_{\beta_{0}}$ is a direct summand of $\mathfrak{M}_{\beta_{0}}$ as $\mathfrak{S}_{1}$-module, it must be generated by $\left(\mathbf{e}_{\beta_{0}}+\left(y_{\sigma^{-1} \circ \beta_{0}}^{p} /\left(a_{\beta_{0}}+b_{\beta_{0}} y_{\sigma^{-1} \circ \beta_{0}}^{p}\right)\right) \mathbf{f}_{\beta_{0}}\right)$. Hence, by [43, Lemma 3.9], we have

$$
\begin{aligned}
\operatorname{deg}_{\beta_{0}}(D)=1-\operatorname{deg}_{\beta_{0}}(A[p] / D) & =\operatorname{deg}\left(\mathfrak{N}_{\beta_{0}} /(1 \otimes \varphi) \varphi^{*}\left(\mathfrak{N}_{\sigma^{-1} \circ \beta_{0}}\right)\right) \\
& =1-v_{p}\left(a_{\beta_{0}}+b_{\beta_{0}} y_{\sigma^{-1} \circ \beta_{0}}^{p}\right)=\delta_{j}
\end{aligned}
$$

for all $D \subseteq A[p]$ with $D \neq H$.

(2) If $\operatorname{deg}_{\beta_{0}}(H)<\delta_{j}$, then we have

$$
\begin{aligned}
v_{u}\left(c_{p^{g}}\right) & =v_{u}\left(a_{\sigma^{-1} \circ \beta_{0}} \cdots a_{\sigma^{-(g-j-2)} \beta_{0}}^{p^{g-j-3}} b_{\sigma^{-(g-j-1)} \circ \beta_{0}}^{p^{g-j-2}} d_{\sigma^{-(g-j)} \circ \beta_{0}}^{p^{g-j-1}} \cdots d_{\sigma^{-g} \beta_{0}}^{p^{g-1}}\right) \\
& =\sum_{i=1}^{g-j-2} p^{i-1}\left(1-\operatorname{deg}_{\sigma^{-i} \circ \beta_{0}}(H)\right)+p^{g-1} \operatorname{deg}_{\beta_{0}}(H) .
\end{aligned}
$$

Hence, all the $p^{g}$-solutions of $y_{\sigma^{-1} \circ \beta_{0}}$ have valuation

$$
v_{p}\left(y_{\sigma^{-1} \circ \beta_{0}}\right)=\frac{1}{p}\left(1-\operatorname{deg}_{\beta_{0}}(H)\right)+\frac{1}{p}\left(\frac{1}{p}+\cdots+\frac{1}{p^{j}}-\operatorname{deg}_{\beta_{0}}(H)\right) .
$$

Hence, we have

$$
\begin{aligned}
& \min \left\{v_{p}\left(a_{\beta_{0}}+b_{\beta_{0}} y_{\sigma^{-1} \circ \beta_{0}}^{p}\right), v_{p}\left(y_{\sigma^{-1} \circ \beta_{0}}^{p} d_{\beta_{0}}\right)\right\} \\
& =v_{p}\left(a_{\beta_{0}}+b_{\beta_{0}} y_{\sigma^{-1} \circ \beta_{0}}^{p}\right)=1-\operatorname{deg}_{\beta_{0}}(H) .
\end{aligned}
$$

As in Case (1) above, $\mathfrak{N}_{\beta_{0}}$ is generated by $\left(\mathbf{e}_{\beta_{0}}+\left(y_{\sigma^{-1} \circ \beta_{0}}^{p} /\left(a_{\beta_{0}}+b_{\beta_{0}} y_{\sigma^{-1} \circ \beta_{0}}^{p}\right)\right) \mathbf{f}_{\beta_{0}}\right)$, and

$$
\operatorname{deg}_{\beta_{0}}(D)=1-v_{p}\left(a_{\beta_{0}}+b_{\beta_{0}} y_{\sigma^{-1} \circ \beta_{0}}^{p}\right)=\operatorname{deg}_{\beta_{0}}(H)
$$

for all $D \subseteq \underline{A}[p]$ with $H \neq D$.

(3) If $\operatorname{deg}_{\beta_{0}}(H)=\delta_{j}$, then

$$
\begin{aligned}
v_{p}\left(c_{p^{g}}\right) & \geqslant \min _{0 \leqslant i \leqslant g-1}\left\{v_{p}\left(a_{\sigma^{-1} \circ \beta_{0}} \cdots a_{\sigma^{-i} \circ \beta_{0}}^{p^{i-1}} b_{\sigma^{-i-1} \circ \beta_{0}}^{p^{i}} d_{\sigma^{-i-2} \circ \beta_{0}}^{p^{i+1}} \cdots d_{\sigma^{g} \circ \beta_{0}}^{p^{g-1}}\right)\right\} \\
& =\sum_{i=1}^{g-j-2} p^{i-1}\left(1-\operatorname{deg}_{\sigma^{-i} \circ \beta_{0}}(H)\right)+p^{g-1}\left(\delta_{j}\right),
\end{aligned}
$$

and all the $p^{g}$-solutions of $y_{\sigma^{-1} \circ \beta_{0}}$ have valuation

$$
v_{p}\left(y_{\sigma^{-1} \circ \beta_{0}}\right) \leqslant \frac{1}{p}\left(1-\delta_{j}\right) .
$$

Hence, we have

$$
\operatorname{deg}_{\beta_{0}}(A[p] / D)=\min \left\{v_{p}\left(a_{\beta_{0}}+b_{\beta_{0}} y_{\sigma^{-1} \circ \beta_{0}}^{p}\right), v_{p}\left(y_{\sigma^{-1} \circ \beta_{0}}^{p} d_{\beta_{0}}\right)\right\} \leqslant 1-\delta_{j},
$$

and thus $\operatorname{deg}_{\beta_{0}}(D) \geqslant \delta_{j}$ for all $D$. This finishes the proof of the lemma. 
Proposition 5.15. Let $W_{\varphi, \eta}$ be a bad stratum of codimension 1 as in Case 2(c) of Definition 5.6, $\beta_{0} \in \mathbb{B}, j \in \mathbb{Z}_{\geqslant 1}$, and let $\delta_{j}$ be as defined there. Then the following hold.

(1) We have

$$
] W_{\varphi, \eta}^{\mathrm{gen}} \mathrm{I}^{\prime} \supset\left\{Q \in\left(W_{\varphi, \eta}^{\mathrm{gen}}\right): \operatorname{deg}_{\beta_{0}}(Q) \in\left(0, \delta_{j}\right) \cup\left(\delta_{j}, 1\right)\right\}
$$

(2) Let $\bar{Q} \in W_{\varphi, \eta}^{\mathrm{gen}}$ be a closed point. Then $] W_{\varphi, \eta}^{\mathrm{gen}}\left[{ }^{\prime} \cap \mathrm{sp}^{-1}(\bar{Q})\right.$ is geometrically connected.

Proof. Part (1) is an immediate consequence of Lemma 5.14 and the definition of $\left(W_{\varphi, \eta}^{\text {gen }}\right)^{\prime}$. By Stamm's theorem [40] (see also [15, Theorem 2.4.1]), we have an isomorphism

$$
\widehat{\mathcal{O}}_{\mathfrak{Y}, \bar{Q}} \simeq \mathcal{O}_{K}\left[\left[x_{\beta_{0}}, y_{\beta_{0}}, z_{2}, \ldots, z_{g}\right]\right] /\left(x_{\beta_{0}} y_{\beta_{0}}-p\right) .
$$

Let $\mathfrak{S}=\operatorname{Spf}\left(\widehat{\mathcal{O}}_{\mathfrak{Y}, \bar{Q}}\right)$. Then, the rigid generic fiber (in the sense of Berthelot)

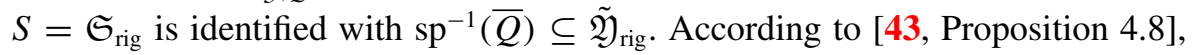
we have $\operatorname{deg}_{\beta_{0}}(H)=\min \left\{1, v_{p}\left(y_{\beta_{0}}(Q)\right)\right\}$ for every rigid point $Q=(A, H) \in S$. Let $T$ be the inverse image of $S$ via the map $\pi_{1, p}: \tilde{\mathfrak{Y}}_{\text {rig }}^{(p)} \rightarrow \tilde{\mathfrak{Y}}_{\text {rig }}$. Then $\left.\pi\right|_{T}: T \rightarrow S$ is a finite étale morphism of rigid analytic spaces. Let $g$ be an analytic function on $T$ such that $\operatorname{deg}_{\beta_{0}}(D)=\min \left\{1, v_{p}\left(g\left(Q^{\prime}\right)\right)\right\}$ for any rigid point $Q^{\prime}=(\underline{A}, H$, $D) \in T \subseteq \tilde{\mathfrak{Y}}_{\text {rig }}^{(p)}$. By the definition of $] W_{\varphi, \eta}^{\text {gen }}[$, we have

$$
] W_{\varphi, \eta}^{\mathrm{gen}}\left[^{\prime} \cap \operatorname{sp}^{-1}(\bar{Q})=S^{|g| \geqslant|p|^{\delta_{j}}},\right.
$$

in the notation of Lemma 5.16 below. Moreover, Lemma 5.14 implies that the assumptions in Lemma 5.16 are satisfied with $u=y_{\beta_{0}}$ and $a=b=\delta_{j}$. Hence, the proposition follows immediately from Lemma 5.16.

LEMMA 5.16. Let $\mathfrak{S}$ be the formal scheme

$$
\mathfrak{S}=\operatorname{Spf}\left(\mathcal{O}_{K}\left[\left[u, v, z_{2}, \ldots, z_{d}\right]\right] /(u v-p)\right),
$$

and let $S=\mathfrak{S}_{\text {rig }}$ be the associated rigid analytic space. Let $\pi: T \rightarrow S$ be a finite étale morphism of rigid analytic spaces. Let $g$ be an analytic function on $T$. Assume that there exist $a, b \in \mathbb{Q}_{>0}$ with $a \in(0,1)$ such that we have

(a) $|g| \leqslant|p|^{b}$ on $\pi^{-1}\left(S^{|p|<|u| \leqslant|p|^{a}}\right)$, where

$$
S^{|p|<|u| \leqslant|p|^{a}}:=\left\{Q \in S:|p|<|u(Q)| \leqslant|p|^{a}\right\},
$$


(b) $|g|=|p|^{b}$ on $\pi^{-1}\left(S^{|p|<|u|<|p|^{a}}\right)$,

(c) $|g|>|p|^{b}$ on $\pi^{-1}\left(S^{|p|^{a}<|u|<1}\right)$.

Then, the admissible open subdomain

$$
S^{|g| \geqslant|p|^{b}}:=\left\{Q \in S:\left|g\left(Q^{\prime}\right)\right| \geqslant|p|^{b} \forall Q^{\prime} \in \pi^{-1}(Q)\right\}
$$

of $S$ is geometrically connected.

Proof. For every integer $N \geqslant 2$, let $S_{N}$ denote the affinoid subdomain of $S$ defined by $|p|^{1-1 / N} \leqslant|u| \leqslant|p|^{1 / N}$ and $\left|z_{i}\right| \leqslant|p|^{1 / N}$ for $2 \leqslant i \leqslant d$. Since $\left\{S_{N}: N \geqslant 3\right\}$ form an admissible open covering of $S$, it suffices to show that $S_{N}^{|g| \geqslant|p|^{b}}:=S_{N} \cap$ $S^{|g| \geqslant|p|^{b}}$ is geometrically connected for $N$ sufficiently large.

Let $A=\mathcal{O}_{K}\left[\left[u, v, z_{2}, \ldots, z_{d}\right]\right] /(u v-p)$. Up to replacing $K$ by a finite extension, we may assume that there exists $\varpi \in \mathcal{O}_{K}$ with $|\varpi|=|p|^{1 / N}$. We put

$$
\begin{gathered}
A_{N}=A\left\langle u_{N}, v_{N}, z_{2, N}, \ldots, z_{d, N}\right\rangle /\left(u-\varpi u_{N}, v-\varpi v_{N},\right. \\
\left.z_{2}-\varpi z_{2, N}, \ldots, z_{d}-\varpi z_{d, N}\right),
\end{gathered}
$$

and $\mathfrak{S}_{N}=\operatorname{Spf}\left(A_{N}\right)$. Then $\mathfrak{S}_{N}$ is an admissible formal scheme over $\mathcal{O}_{K}$, and $\mathfrak{S}_{N, \text { rig }}=S_{N}$. Up to replacing $N$ by a multiple, we may assume that $a=m / N$, $b=n / N$ with $m, n \in \mathbb{Z}_{\geqslant 1}$ and $1<m<N-1$. Let $\tilde{\mathfrak{S}}_{N}$ be the $p$-adic completion of the maximal $\mathcal{O}_{K}$-flat closed subscheme of the admissible blow-up $\operatorname{Proj}\left(A_{N}\left[X_{1}, X_{2}\right] /\left(X_{1} u_{N}-X_{2} \varpi^{m-1}\right)\right)$ of $\mathfrak{S}_{N}$ along the ideal $I=\left(u_{N}, \varpi^{m-1}\right)$. By Raynaud's theory, the natural map $\tilde{\mathfrak{S}}_{N} \rightarrow \mathfrak{S}_{N}$ induces an isomorphism on rigid generic fibers. Let $\mathfrak{U}_{N, 1}$ be the open formal scheme of $\tilde{\mathfrak{S}}_{N}$ defined by $X_{1} \neq 0$, and let $\mathfrak{U}_{N, 2}$ be that defined by $X_{2} \neq 0$. Then $\mathfrak{U}_{1 \text {,rig }}$ (respectively, $\mathfrak{U}_{2, \text { rig }}$ ) is the admissible open subset of $\mathfrak{X}_{N \text {,rig }}$ defined by $|p|^{1-1 / N} \leqslant|u| \leqslant|p|^{a}$ (respectively, $\left.|p|^{a} \leqslant|u| \leqslant|p|^{1 / N}\right)$. Then $x_{2}=X_{2} / X_{1}$ and $x_{1}=X_{1} / X_{2}$ are respectively defined on $\mathfrak{U}_{N, 1}$ and $\mathfrak{U}_{N, 2}$, and $x_{2}=x_{1}^{-1}$ on $\mathfrak{U}_{N, 1} \cap \mathfrak{U}_{N, 2}$. We have $u_{N}=\varpi^{m-1} x_{2}$ on $\mathfrak{U}_{N, 1}$, and $v_{N}=\frac{p}{\varpi^{m+1}} x_{1}$ on $\mathfrak{U}_{N, 2}$. Then, the special fiber of $\tilde{\mathfrak{S}}_{N}$, denoted by $\tilde{S}_{N, 0}$, is geometrically reduced, and it consists of three geometrically irreducible components, $C_{0}, C_{\infty}$, and $C_{1}$, which are all smooth of dimension $d$ and defined respectively by $x_{2}=0, x_{1}=0$ (that is, $x_{2}=\infty$ ), and $u_{N}=v_{N}=0$. Let $U_{N, i}$ for $i=1,2$ denote the open subset of $\tilde{S}_{N, 0}$ corresponding to $\mathfrak{U}_{N, i}$. Then we have $U_{N, 1}=\tilde{S}_{N, 0}-C_{\infty}$ and $U_{N, 2}=\tilde{S}_{N, 0}-C_{0}$.

Let $\tilde{\mathfrak{T}}_{N}$ be the normalization of $\tilde{\mathfrak{S}}_{N}$ in $T$. Then $\tilde{\mathfrak{T}}_{N}$ is a formal model of $T_{N}:=$ $\pi^{-1}\left(\mathfrak{S}_{N, \text { rig }}\right)$, and $\pi$ extends to a finite map $\pi: \tilde{\mathfrak{T}}_{N} \rightarrow \tilde{\mathfrak{S}}_{N}$ of formal schemes. We put $\mathfrak{V}_{N, i}=\pi^{-1}\left(\mathfrak{U}_{N, i}\right)$. Consider the rigid analytic function $\tilde{g}=g / \varpi^{n}$. We have 
$|\tilde{g}| \leqslant 1$ on $\mathfrak{V}_{N, 1 \text {,rig }}$ by Condition (a). Hence, $\tilde{g}$ descends to a global section on $\mathfrak{V}_{N, 1}$. Let $V_{N, 1}$ be the reduced special fiber of $\mathfrak{V}_{N, 1}$, and let $\bar{g}$ denote the image of $\tilde{g}$ in $\Gamma\left(V_{N, 1}, \mathcal{O}_{V_{N, 1}}\right)$ by reduction. Let $Z \subseteq V_{N, 1}$ denote the closed subscheme defined by the vanishing of $\bar{g}$. Note that $(Z)$ is the subdomain of $Y_{N}$ such that $|g|<|p|^{b}$ by our construction of $Z$ and Condition (c). Therefore, we have

$$
S_{N}^{|g| \geqslant|p|^{b}}=\mathfrak{S}_{N, \text { rig }}-\pi((Z))=\left(\tilde{S}_{N, 0}-\pi(Z)\right) .
$$

By [2, Proposition 4.3], $S_{N}^{|g| \geqslant|p|^{b}}$ is geometrically connected if and only if $\tilde{S}_{N, 0}-$ $\pi(Z)$ is geometrically connected. Since $\pi$ is finite, $\pi(Z)$ is a closed subscheme of $U_{N, 1}$. By Condition (b), $\pi(Z)$ has no intersection with $C_{0}$ (since $\left(C_{0}\right)$ is the admissible open of $\mathfrak{X}_{N \text {,rig }}$ defined by $\left.|p|^{1-1 / N} \leqslant|u|<|p|^{a}\right)$. Thus $\pi(Z)$ is a closed subset contained in $U_{N, 1}-C_{0}=C_{1}-C_{0}-C_{\infty}$. Since $C_{1}-C_{0}-C_{\infty}$ is smooth and irreducible of dimension $d, \pi(Z)$ must have dimension $<d$. Hence, $\tilde{S}_{N, 0}-\pi(Z)$ is geometrically connected. This finishes the proof of the lemma.

\section{Gluing}

Let $\underline{A}$ be a Hilbert-Blumenthal abelian variety. For any point $P$ of $A[p]$ and $\mathfrak{p} \in \mathbb{S}$, write $P=P^{\mathfrak{p}} \times P_{\mathfrak{p}}$, where $P^{\mathfrak{p}} \in A[p / \mathfrak{p}]$ and $P_{\mathfrak{p}} \in A[\mathfrak{p}]$. Throughout this section, for a point of $Z_{K}$, we will replace our usual notation of $(\underline{A}, P)$ by $(\underline{\underline{A}}$, $\left.P_{\mathfrak{p}}\right)$, where $P_{\mathfrak{p}}$ is as above, and $\underline{\underline{A}}=\left(\underline{A}, P^{\mathfrak{p}}\right)$. For a point $Q$ of $\underline{A}$ of finite order, we denote by $(Q)$ the $\mathcal{O}_{L}$-module generated by $Q$ inside $A$.

We begin by introducing some auxiliary varieties. Let us fix $\mathfrak{n} \mid N$, an ideal of $\mathcal{O}_{L}$, as well as an $\mathcal{O}_{L}$-generator $\zeta_{\mathfrak{n}}$ of $\left(\mathbb{G}_{m} \otimes \mathfrak{d}_{L}^{-1}\right)[\mathfrak{n}]$. Recall the choice of $\zeta_{\mathfrak{p}}$ in Section 1.10. For a Hilbert-Blumenthal abelian variety $\underline{A}$, consider the $\mathcal{O}_{L}$-linear Weil pairing $\langle-,-\rangle_{\mathfrak{n}}: A[\mathfrak{n}] \times A[\mathfrak{n}] \rightarrow\left(\mathbb{G}_{m} \otimes \mathfrak{d}_{L}^{-1}\right)[\mathfrak{n}]$.

Let $Z_{K}^{\mathfrak{n}}$ be the scheme which classifies tuples $\left(\underline{\underline{A}}, P_{\mathfrak{p}}, P_{\mathfrak{n}}, Q_{\mathfrak{n}}\right)$ over $K$-schemes, where $\left(\underline{\underline{A}}, P_{\mathfrak{p}}\right)$ is classified by $Z_{K}$, and $P_{\mathfrak{n}}, Q_{\mathfrak{n}}$ are points of maximal order in $A[\mathfrak{n}]$ satisfying $\left(P_{\mathfrak{n}}\right)=\left(P_{N}\right) \cap \underline{A}[\mathfrak{n}]$, where $P_{N}$ is the point giving the level $N$ structure included in the notation $\underline{\underline{A}}$, and such that $\left\langle P_{\mathfrak{n}}, Q_{\mathfrak{n}}\right\rangle_{\mathfrak{n}}=\zeta_{\mathfrak{n}}$. Let $\tilde{\mathfrak{Z}}_{\text {rig }}^{\mathfrak{n}}$ denote the toroidal compactification of $Z_{K}^{\mathfrak{n}}$ obtained using our fixed choice of collection of cone decompositions, with the same notation used for the associated rigid analytic variety.

Given $\mathfrak{p} \in \mathbb{S}$, let $Z_{K}^{\mathfrak{p}, \underline{\mathfrak{n}}}$ be the scheme which classifies tuples $\left(\underline{\underline{A}}, P_{\mathfrak{p}}, Q_{\mathfrak{p}}, P_{\mathfrak{n}}\right.$, $\left.Q_{\mathfrak{n}}\right)$ over $K$-schemes, where $\left(\underline{\underline{A}}, P_{\mathfrak{p}}, P_{\mathfrak{n}}, Q_{\mathfrak{n}}\right)$ is classified by $Z_{K}^{\mathfrak{n}}$, and $Q_{\mathfrak{p}}$ is a point of maximal order in $A[\mathfrak{p}]$ such that $\left\langle P_{\mathfrak{p}}, Q_{\mathfrak{p}}\right\rangle_{\mathfrak{p}}=\zeta_{\mathfrak{p}}$. Let $\tilde{\mathfrak{Z}}_{\text {rig }}^{\mathfrak{p}, \mathfrak{n}}$ denote the toroidal compactification of $Z_{K}^{\mathfrak{p}, \mathfrak{n}}$ with the same notation used for the associated rigid analytic variety. 
For any $j_{\mathfrak{p}} \in \mathcal{O}_{L} / \mathfrak{p}$, there is a finite flat morphism

$$
\pi_{2, j_{\mathfrak{p}}}: \tilde{\mathfrak{Z}}_{\text {rig }}^{\mathfrak{p}, \underline{\mathfrak{n}}} \rightarrow \tilde{\mathfrak{Z}}_{\text {rig }}^{\mathfrak{n}},
$$

defined by $\pi_{2, j_{\mathfrak{p}}}\left(\underline{\underline{A}}, P_{\mathfrak{p}}, Q_{\mathfrak{p}}, P_{\mathfrak{n}}, Q_{\mathfrak{n}}\right)=\left(\underline{\underline{A}} /\left(j_{\mathfrak{p}} P_{\mathfrak{p}}+Q_{\mathfrak{p}}\right), \overline{P_{\mathfrak{p}}}, \overline{P_{\mathfrak{n}}}, \overline{Q_{\mathfrak{n}}}\right)$ on the noncuspidal part, where overline denotes image in the quotient. We also define

$$
\pi_{1, \mathfrak{p}}: \tilde{\mathfrak{Z}}_{\text {rig }}^{\mathfrak{p}, \mathfrak{n}} \rightarrow \tilde{\mathfrak{Z}}_{\text {rig }}^{\mathfrak{n}},
$$

via $\pi_{1, \mathfrak{p}}\left(\underline{\underline{A}}, P_{\mathfrak{p}}, Q_{\mathfrak{p}}, P_{\mathfrak{n}}, Q_{\mathfrak{n}}\right)=\left(\underline{\underline{A}}, P_{\mathfrak{p}}, P_{\mathfrak{n}}, Q_{\mathfrak{n}}\right)$. Similarly, for any $j_{\mathfrak{n}} \in \mathcal{O}_{L} / \mathfrak{n}$, we define

$$
\pi_{2, j_{\mathfrak{n}}}: \tilde{\mathfrak{Z}}_{\text {rig }}^{\mathfrak{n}} \rightarrow \tilde{\mathfrak{Z}}_{\text {rig }}
$$

by $\pi_{2, j_{\mathfrak{n}}}\left(\underline{\underline{A}}, P_{\mathfrak{p}}, P_{\mathfrak{n}}, Q_{\mathfrak{n}}\right)=\left(\underline{\underline{A}} /\left(j_{\mathfrak{n}} P_{\mathfrak{n}}+Q_{\mathfrak{n}}\right), \overline{P_{\mathfrak{p}}}\right)$. We also define

$$
\pi_{1, \mathfrak{n}}: \tilde{\mathfrak{Z}}_{\text {rig }}^{\mathfrak{n}} \rightarrow \tilde{\mathfrak{Z}}_{\text {rig }}
$$

via $\pi_{1, \mathfrak{n}}\left(\underline{\underline{A}}, P_{\mathfrak{p}}, P_{\mathfrak{n}}, Q_{\mathfrak{n}}\right)=\left(\underline{\underline{A}}, P_{\mathfrak{p}}\right)$. Finally, we define

$$
w_{\mathfrak{p}}: \tilde{\mathfrak{Z}}_{\text {rig }}^{\mathfrak{n}} \rightarrow \tilde{\mathfrak{Z}}_{\text {rig }}^{\mathfrak{n}},
$$

given by sending $\left(\underline{A}, P_{\mathfrak{p}}, P_{\mathfrak{n}}, Q_{\mathfrak{n}}\right)$ to $\left(\underline{A} /\left(P_{\mathfrak{p}}\right), \overline{Q_{\mathfrak{p}}}, \overline{P_{\mathfrak{n}}}, \overline{Q_{\mathfrak{n}}}\right)$, for any choice of a point of maximal order $Q_{\mathfrak{p}} \in A[\mathfrak{p}]$ satisfying $\left\langle P_{\mathfrak{p}}, Q_{\mathfrak{p}}\right\rangle_{\mathfrak{p}}=\zeta_{\mathfrak{p}}$.

Definition 6.1. Let $\mathfrak{p} \in \mathbb{S}$. We define $\mathbb{I}_{\mathfrak{p}}^{*}$ to be the interval $\left(\sum_{i=1}^{f_{\mathfrak{p}}-1} 1 / p^{i}, 1\right)$ if $f_{\mathfrak{p}}>1$, and $(0,1)$ if $f_{\mathfrak{p}}=1$.

Definition 6.2. Recall $\tilde{\mathfrak{Y}}_{\text {rig }}^{|\tau| \leqslant 1}$ which was introduced in [19, Section 5]. It is an admissible open in $\tilde{\mathfrak{Y}}_{\text {rig }}$ whose points are those $Q=(\underline{A}, C)$ such that $\left|\tau(\bar{Q}) \cap \mathbb{B}_{\mathfrak{p}}\right| \leqslant 1$, for all $\mathfrak{p}$, where $\tau(\bar{Q})$ is defined in (2.4.1). We define $\tilde{\mathfrak{Z}}_{\text {rig }}^{|\tau| \leqslant 1}=$ $\alpha^{-1}\left(\tilde{\mathfrak{Y}}_{\text {rig }}^{|\tau| \leqslant 1}\right)$.

The following lemma is crucial for the gluing process and was essentially proved in [19].

LEMMA 6.3. Let $\underline{I}$ be a multiset of intervals as in Definition 2.2, such that $I_{\mathfrak{p}}=\mathbb{I}_{\mathfrak{p}}^{*}$ for some $\mathfrak{p} \mid p$.

(1) If $(\underline{A}, P) \in \alpha^{-1}(\Sigma \underline{I})$, then, for any nonzero point $Q_{\mathfrak{p}}$ of $A[\mathfrak{p}]$, we have

$$
\left(A, P^{\mathfrak{p}} \times Q_{\mathfrak{p}}\right) \in \alpha^{-1}(\Sigma \underline{I}) .
$$


(2) For all $j_{\mathfrak{p}} \in \mathcal{O}_{L} / \mathfrak{p}$, we have the following inclusion inside $\tilde{\mathfrak{Z}}_{\text {rig }}^{\underline{\mathfrak{p}}, \mathfrak{n}}$ :

$$
\pi_{1, \mathfrak{p}}^{-1} \pi_{1, \mathfrak{n}}^{-1} \alpha^{-1}(\Sigma) \subset \pi_{2, j_{\mathfrak{p}}}^{-1} \pi_{1, \mathfrak{n}}^{-1} \alpha^{-1}(\Sigma)
$$

Proof. The first statement follows directly from [19, Lemma 5.9, Corollary 7.9] (keeping in mind Remark 2.3). The second statement follows from Proposition 5.9 .

In the following, we will prove two connectivity results which are essential for the gluing process. Given $\mathcal{U} \subset \tilde{\mathfrak{Y}}_{\text {rig }}, I \subset[0,1]$, and $\beta \in \mathbb{B}$, we define an admissible open subset of $\mathcal{U}$,

$$
\mathcal{U} I_{\beta}:=\left\{Q \in \mathcal{U}: \operatorname{deg}_{\beta}(Q) \in I\right\}
$$

We first prove a preliminary result which follows directly from the study of the geometry of $\tilde{\bar{Y}}$ (the special fiber of $\tilde{Y}$ ) conducted in [15]. For $W \subset \tilde{\bar{Y}}$, we denote its Zariski closure by $W^{c l}$.

LEMMA 6.4. Let $W_{\varphi, \eta}$ be a stratum in $\tilde{\bar{Y}}$, and let $\left(\varphi^{\prime}, \eta^{\prime}\right)$ be another admissible pair with $\varphi^{\prime} \supseteq \varphi, \eta^{\prime} \supseteq \eta$.

(1) Let $W$ be an irreducible component of $W_{\varphi, \eta}$. There exists an irreducible component $V$ of $W_{\varphi^{\prime}, \eta^{\prime}}$, such that $V \subset W^{c l}$.

(2) Let $V$ be an irreducible component of $W_{\varphi^{\prime}, \eta^{\prime}}$. There exists an irreducible component $W$ of $W_{\varphi, \eta}$ such that $V \subset W^{c l}$.

Proof. Part (2) is immediate: since $V \subset W_{\varphi^{\prime}, \eta^{\prime}} \subset W_{\varphi, \eta}^{c l}$, it follows that $V^{c l}$ lies inside an irreducible component of $W_{\varphi, \eta}^{c l}$.

For part (1), we argue as follows. Let $W$ be an irreducible component of $W_{\varphi, \eta}$. By [15, Theorem 2.6.13], $W^{c l}$ contains a point in $W_{\mathbb{B}, \mathbb{B}}$. Since $W_{\mathbb{B}, \mathbb{B}}$ lies inside every closed stratum, it follows that $W^{c l}$ intersects $V^{c l}$, where $V$ is an irreducible component of $W_{\varphi^{\prime}, \eta^{\prime}}$. By part (2) of the lemma, there is an irreducible component $W_{1}$ of $W_{\varphi, \eta}$ such that $V \subset W_{1}^{c l}$. The nonsingularity of the strata implies that no two distinct irreducible components of $W_{\varphi, \eta}^{c l}$ meet, and, hence, we must have $W_{1}=W$. This ends the proof.

The following general definition includes, as examples, two regions in $\tilde{\mathfrak{Y}}_{\text {rig }}$ for which we want to prove connectivity results.

DEFINITION 6.5. Assume that for any nowhere-étale stratum $W_{\varphi, \eta}$ of $\tilde{\bar{Y}}$ of codimension 0 or 1 , we are given a Zariski open dense subset $W_{\varphi, \eta}^{0} \subset W_{\varphi, \eta}$, and 
an admissible open subset $\mathcal{R}_{W_{\varphi, \eta}} \subset \mathrm{sp}^{-1}\left(W_{\varphi, \eta}^{0}\right)$. Given such a collection $\mathcal{R}$, we define

$$
\Sigma_{\mathcal{R}}:=\bigcup_{(\varphi, \eta)} \mathcal{R}_{W_{\varphi, \eta}},
$$

where $(\varphi, \eta)$ runs over all admissible pairs with $W_{\varphi, \eta}$ nowhere étale and of codimension 0 or 1 . For any irreducible component $W \subset W_{\varphi, \eta}$, we set

$$
\begin{gathered}
W^{0}:=W_{\varphi, \eta}^{0} \cap W, \\
\mathcal{R}_{W}:=\mathcal{R}_{W_{\varphi, \eta}} \cap \mathrm{sp}^{-1}(W) .
\end{gathered}
$$

We assume that the following conditions hold.

(1) If $W \subset W_{\varphi, \eta}$ is an irreducible component of a nowhere-étale stratum of codimension 0 , then $\mathcal{R}_{W}=\mathrm{sp}^{-1}\left(W^{0}\right)$.

(2) Let $V \subset W_{\varphi, \eta}$ be an irreducible component of a nowhere-étale stratum of codimension 1 , and let $\ell(\varphi) \cap \eta=\{\beta\} \subset \mathbb{B}_{\mathfrak{p}}$. Then, if $\eta_{\mathfrak{p}}:=\eta \cap \mathbb{B}_{\mathfrak{p}} \neq \mathbb{B}_{\mathfrak{p}}$, we have

$$
\begin{aligned}
& \mathcal{R}_{V}(0, \delta)_{\beta}=\mathrm{sp}^{-1}\left(V^{0}\right)(0, \delta)_{\beta}, \\
& \mathcal{R}_{V}(\epsilon, 1)_{\beta}=\mathrm{sp}^{-1}\left(V^{0}\right)(\epsilon, 1)_{\beta},
\end{aligned}
$$

for some $0<\epsilon, \delta<1$. If $\eta_{\mathfrak{p}}=\mathbb{B}_{\mathfrak{p}}$, then we only require the second condition.

(3) Let $V$ be as in (2), $Q \in \mathcal{R}_{V}$, and $\bar{Q}=\operatorname{sp}(Q)$. Then, $\operatorname{sp}^{-1}(\bar{Q}) \cap \mathcal{R}_{V}$ is connected, and $\operatorname{deg}_{\beta}$ takes values arbitrarily close to 0 and 1 on it (if $\eta_{\mathfrak{p}}=\mathbb{B}_{\mathfrak{p}}$, we only require that $\operatorname{deg}_{\beta}$ can take values arbitrarily close to 1 ).

Lemma 6.6. Fix $\mathfrak{p} \in \mathbb{S}$. Assume that the collection $\mathcal{R}$ is as in Definition 6.5.

(1) Let $Q \in \Sigma_{\mathcal{R}} \cap \mathrm{sp}^{-1}\left(W_{\varphi, \eta}\right)$, where $W_{\varphi, \eta}$ is a nowhere-étale stratum of codimension 0 . Then, there is a connected subset $C \subset \Sigma_{\mathcal{R}}$ which contains $Q$, and such that $C \cap \operatorname{sp}^{-1}\left(W_{\varphi, \eta \cup\{\beta\}}\right) \neq \emptyset$, for any $\beta \notin \eta_{\mathfrak{p}}$.

(2) Let $Q \in \Sigma_{\mathcal{R}} \cap \operatorname{sp}^{-1}\left(W_{\varphi, \eta}\right)$, where $W_{\varphi, \eta}$ is a nowhere-étale stratum of codimension 0 such that $\varphi_{\mathfrak{p}} \neq \mathbb{B}_{\mathfrak{p}}$. Then, there is a connected subset $C \subset \Sigma_{\mathcal{R}}$ which contains $Q$, and such that $C \cap \operatorname{sp}^{-1}\left(W_{\varphi \cup\{\beta\}, \eta}\right) \neq \emptyset$, for any $\beta \notin \varphi_{\mathfrak{p}}$.

(3) Let $Q \in \Sigma_{\mathcal{R}} \cap \operatorname{sp}^{-1}\left(W_{\varphi, \eta}\right)$, where $W_{\varphi, \eta}$ is a nowhere-étale stratum of codimension 1 such that $\eta_{\mathfrak{p}} \neq \mathbb{B}_{\mathfrak{p}}$ (respectively, $\varphi_{\mathfrak{p}} \neq \mathbb{B}_{\mathfrak{p}}$ ). Then, there is a connected $C \subset \Sigma_{\mathcal{R}}$ containing $Q$, such that $C \cap \operatorname{sp}^{-1}\left(W_{\varphi-\{\sigma \circ \beta\}, \eta}\right) \neq \varnothing$ (respectively, $\left.C \cap \operatorname{sp}^{-1}\left(W_{\varphi, \eta-\{\beta\}}\right) \neq \emptyset\right)$, where $\{\beta\}=\ell(\varphi) \cap \eta$. 
The lemma immediately implies the following.

COROllary 6.7. Let $\mathcal{R}$ be as in Definition 6.5. Every connected component of $\Sigma_{\mathcal{R}}$ intersects $\mathrm{sp}^{-1}\left(W_{\mathbb{B}, \emptyset}\right)$ as well as $\mathrm{sp}^{-1}\left(W_{\emptyset, \mathbb{B}}\right)$. Furthermore, given $\mathfrak{p} \in \mathbb{S}$, every connected component of $\Sigma_{\mathcal{R}}$ intersects $\mathrm{sp}^{-1}\left(W_{\varphi, \eta}\right)$, where $W_{\varphi, \eta}$ is any codimension-1 stratum satisfying $\varphi_{\mathfrak{q}}=\mathbb{B}_{\mathfrak{q}}, \eta_{\mathfrak{q}}=\emptyset$, for $\mathfrak{q} \neq \mathfrak{p}$, and $\eta_{\mathfrak{p}}=\mathbb{B}_{\mathfrak{p}}$, $\left|\varphi_{\mathfrak{p}}\right|=1$ (respectively, $\varphi_{\mathfrak{p}}=\mathbb{B}_{\mathfrak{p}},\left|\eta_{\mathfrak{p}}\right|=1$ ).

Proof of Lemma 6.6. We begin by proving (1). Let $W_{1}$ be an irreducible component of $W_{\varphi, \eta}$ containing $\bar{Q}$. By Definition 6.5, $Q \in \mathrm{sp}^{-1}\left(W_{1}^{0}\right)$. By Lemma 6.4 there is an irreducible component $V$ of $W_{\varphi, \eta \cup\{\beta\}}$ which lies inside $W_{1}^{c l}$, and an irreducible component of $W_{\varphi-\{\sigma \circ \beta\}, \eta \cup\{\beta\}}$, say $W_{2}$, such that $V$ lies inside $W_{2}^{c l}$. Note that $\beta \in \eta^{c} \subset \ell(\varphi)$, and hence $W_{2}$ has codimension 0 . We set $U=W_{1} \cup V \cup W_{2}$. Then,

$$
\tilde{\bar{Y}}-U=\bigcup_{Z} Z
$$

where $Z$ runs over all irreducible components of all closed codimension-0 strata different from $W_{1}^{c l}$ and $W_{2}^{c l}$.

It follows that $U$ is Zariski open in $\tilde{\bar{Y}}$. Hence, so is $U^{0}:=W_{1}^{0} \cup V^{0} \cup W_{2}^{0}$. In particular, for any rational $0<\epsilon<1$, the region

$$
U^{0}[\epsilon, 1]_{\beta}=\operatorname{sp}^{-1}\left(W_{1}^{0}\right) \cup \operatorname{sp}^{-1}\left(V^{0}\right)[\epsilon, 1]_{\beta}
$$

is quasi-compact (noting that $\operatorname{deg}_{\beta}$ is 1 on $\mathrm{sp}^{-1}\left(W_{1}\right)$ and 0 on $\mathrm{sp}^{-1}\left(W_{2}\right)$, and in $(0,1)$ on $\left.\operatorname{sp}^{-1}(V)\right)$. Note that $U^{0}[1,1]_{\beta}=\operatorname{sp}^{-1}\left(W_{1}^{0}\right)$ is connected since $W_{1}^{0}$ is dense in $W_{1}$. We claim that $U^{0}[\epsilon, 1]_{\beta}$ is connected as well. Assume not. Then, it must have a connected component $D$ which does not intersect $U^{0}[1,1]_{\beta}$. Since $D$ is quasi-compact, we can use the maximum modulus principle to deduce that

$$
D \subset U^{0}\left[\epsilon, \epsilon_{0}\right]_{\beta},
$$

for some $\epsilon_{0}<1$. Let $P \in D$ with specialization $\bar{P}$. Let $\mathcal{A}:=\operatorname{sp}^{-1}(\bar{P})[\epsilon, 1)_{\beta}$. Since $\bar{P} \in W_{\varphi, \eta \cup\{\beta\}}$, we deduce by Stamm's theorem, presented in [15, Theorem 2.4.1], that

$$
\operatorname{sp}^{-1}(\bar{P}) \cong\left\{\left(x_{\gamma}\right)_{\gamma \in \mathbb{B}}: v\left(x_{\gamma}\right) \geqslant 0 \forall \gamma \in \mathbb{B}-\{\beta\} ; 0 \leqslant v\left(x_{\beta}\right) \leqslant 1\right\},
$$

where, for any $R \in \operatorname{sp}^{-1}(\bar{P})$, we have $\operatorname{deg}_{\beta}(R)=1-v_{\beta}(Q)=1-v\left(x_{\beta}(R)\right)$ by Remark 2.3. Therefore, $\mathcal{A}$ is a connected subset which contains $P$ and lies entirely in $U^{0}[\epsilon, 1]_{\beta}$. It follows that $\mathcal{A} \subset D$, which contradicts ( $\left.\dagger\right)$, as $\operatorname{deg}_{\beta}$ takes values arbitrarily close to 1 on $\mathcal{A}$. Now, taking $\epsilon$ as in part (2) of Definition 6.5 
(and remembering that $Q \in \mathrm{sp}^{-1}\left(W_{1}^{0}\right)$ ), we find that $C=U^{0}[\epsilon, 1]_{\beta}$ serves as the desired connected subset.

Part (2) of the lemma can be proven exactly as above. We now turn to part (3) of the lemma. Let $V$ be an irreducible component of $W_{\varphi, \eta}$ which contains $\bar{Q}$. As above, let $W_{1}$ be an irreducible component of $W_{\varphi, \eta-\{\beta\}}$ whose Zariski closure contains $V$. Repeating the above argument, we find that, for an appropriate choice of $\epsilon, U^{0}[\epsilon, 1]_{\beta}:=\operatorname{sp}^{-1}\left(W_{1}^{0}\right) \cup \mathrm{sp}^{-1}\left(V^{0}\right)[\epsilon, 1)_{\beta}$ is connected and lies entirely inside $\mathcal{R}_{\Sigma}$. By Assumptions (2) and (3) in Definition 6.5, $\mathrm{sp}^{-1}(\bar{Q}) \cap \mathcal{R}_{\Sigma}$ is connected and $\operatorname{deg}_{\beta}$ takes values arbitrarily close to 1 on it. Since $\operatorname{sp}^{-1}(\bar{Q}) \subset \operatorname{sp}^{-1}\left(V^{0}\right)$, it follows that $\operatorname{sp}^{-1}(\bar{Q}) \cap \mathcal{R}_{\Sigma}$ intersects $U^{0}[\epsilon, 1]_{\beta}$. This shows that $Q$ lies in $C$, the connected component of $\mathcal{R}_{\Sigma}$ containing $U^{0}[\epsilon, 1]_{\beta}$, which clearly intersects $\operatorname{sp}^{-1}\left(W_{\varphi, \eta-\{\sigma \circ \beta\}}\right)$. The other statement in part (3) of the lemma can be proved in exactly the same way.

Proposition 6.8. Let $\Sigma$ be the region defined in Definition 5.6. Fix $\mathfrak{p} \in \mathbb{S}$.

(1) Every connected component of $\Sigma$ contains $\mathrm{sp}^{-1}(W)$, where $W$ is an irreducible component of $W_{\mathbb{B}, \emptyset}$, as well as $\mathrm{sp}^{-1}(V)$, where $V$ is an irreducible component of $W_{\mathbb{B},\{\beta\}}$, for some $\beta \in \mathbb{B}_{\mathfrak{p}}$.

(2) Let $T_{1}, T_{2} \subset \mathbb{S}$. Every connected component of $w_{T_{1}}^{-1}(\Sigma) \cap w_{T_{2}}^{-1}(\Sigma)$ intersects a region of the form $\tilde{\mathfrak{Y}}_{\mathrm{rig}}^{|\tau| \leqslant 1} \underline{I}$, where $\underline{I}$ is a multiset of intervals satisfying $I_{\mathfrak{p}}=\mathbb{I}_{\mathfrak{p}}^{*}$ given in Definition 6.1.

Proof. We consider the following two choices for $\mathcal{R}:=\left\{\mathcal{R}_{V}\right\}$ as in Definition 6.5: for the first one, we take

$$
W_{\varphi, \eta}^{0}:=W_{\varphi, \eta}^{\text {gen }} \quad \text { and } \quad \mathcal{R}_{W_{\varphi, \eta}}:=\left(W_{\varphi, \eta}^{\text {gen }}\right)^{\prime},
$$

so that $\Sigma_{\mathcal{R}}=\Sigma$; the second choice for $\mathcal{R}:=\left\{\mathcal{R}_{V}\right\}$ is given by

$$
\begin{aligned}
W_{\varphi, \eta}^{0}: & =w_{T_{1}}^{-1}\left(W_{w_{T_{1}}(\varphi, \eta)}^{\mathrm{gen}}\right) \cap w_{T_{2}}^{-1}\left(W_{w_{T_{2}}(\varphi, \eta)}^{\mathrm{gen}}\right) \\
\mathcal{R}_{W_{\varphi, \eta}}: & =w_{T_{1}}^{-1}\left(\left(W_{w_{T_{1}}(\varphi, \eta)}^{\mathrm{gen}}\right)^{\prime}\right) \cap w_{T_{2}}^{-1}\left(\left(W_{w_{T_{2}}(\varphi, \eta)}^{\mathrm{gen}}\right)^{\prime}\right),
\end{aligned}
$$

so that $\Sigma_{\mathcal{R}}=w_{T_{1}}^{-1}(\Sigma) \cap w_{T_{2}}^{-1}(\Sigma)$. Here, we refer to Subsection 2.4 for the notation $W_{\varphi, \eta}$. To prove the proposition, we first need to show that the two collections $\mathcal{R}$ presented above satisfy the conditions given in Definition 6.5. This will be done in Lemma 6.9. Assume this is the case for the rest of this proof. Then, applying Corollary 6.7 to the first choice of $\mathcal{R}$ (6.8.1), we find that every connected component of $\Sigma$ intersects $\operatorname{sp}^{-1}\left(W_{\mathbb{B}, \emptyset}\right)$ as well as $\operatorname{sp}^{-1}\left(W_{\mathbb{B},\{\beta\}}\right)$, for some $\beta \in \mathbb{B}_{\mathfrak{p}}$. Since $\operatorname{sp}^{-1}\left(W_{\mathbb{B}, \emptyset}\right) \subset \Sigma \cap \mathcal{V}_{\text {can }}$, it follows that every connected component of $\Sigma$ 
contains a connected component of $\mathrm{sp}^{-1}\left(W_{\mathbb{B}, \emptyset}\right)$. Nonsingularity of strata implies that every connected component of $\operatorname{sp}^{-1}\left(W_{\mathbb{B}, \emptyset}\right)$ is of the form $\operatorname{sp}^{-1}(W)$, where $W$ is an irreducible component of $W_{\mathbb{B}, \emptyset}$. An exactly similar argument, using the fact that $\operatorname{sp}^{-1}\left(W_{\mathbb{B},\{\beta\}}\right) \subset \Sigma \cap \mathcal{V}_{\text {can }}$, proves the second statement in part (1) of the lemma.

For part (2) of the lemma, we use the second choice of $\mathcal{R}$ (6.8.2). By Corollary 6.7, every connected component of $\Sigma_{\mathcal{R}}$ intersects $\operatorname{sp}^{-1}\left(W_{\varphi, \eta}\right)$, where $W_{\varphi, \eta}$ is a codimension-1 stratum satisfying $\varphi_{\mathfrak{q}}=\mathbb{B}_{\mathfrak{q}}, \eta_{\mathfrak{q}}=\emptyset$, for $\mathfrak{q} \neq \mathfrak{p}$, and $\left|\varphi_{\mathfrak{p}}\right|=1, \eta_{\mathfrak{p}}=\mathbb{B}_{\mathfrak{p}}$. To prove part (2) of the lemma, it is enough to show that

$$
\operatorname{sp}^{-1}\left(W_{\varphi, \eta}\right) \cap \Sigma_{\mathcal{R}} \subset \tilde{\mathfrak{Y}}_{\text {rig }}^{|\tau| \leqslant 1} \underline{I},
$$

where $\underline{I}$ is a multiset of intervals with $I_{\mathfrak{p}}=\mathbb{I}_{\mathfrak{p}}^{*}$, and $I_{\mathfrak{q}}=\left\{n_{\mathfrak{q}}\right\}$, with an integer $n_{\mathfrak{q}} \in\left[0, f_{\mathfrak{q}}\right]$, for all $\mathfrak{q} \neq \mathfrak{p}$. By [15, Corollary 2.3.4], on $W_{\varphi, \eta}$, we have $\tau_{\mathfrak{q}}=\emptyset$, and $\left|\tau_{\mathfrak{p}}\right|=1$, which implies that $\operatorname{sp}^{-1}\left(W_{\varphi, \eta}\right) \subset \tilde{\mathfrak{Y}}_{\text {rig }}^{|\tau| \leqslant 1}$. Let $\underline{I}$ be the smallest multiset of intervals satisfying $(\dagger \dagger)$. By the definition of partial degrees on $\operatorname{sp}^{-1}\left(W_{\varphi, \eta}\right)$, it follows that, for $\mathfrak{q} \neq \mathfrak{p}, I_{\mathfrak{q}}$ must be a singleton consisting of an integer in $\left[0, f_{\mathfrak{q}}\right]$. Finally, it follows from the definition of $\Sigma$ that $I_{\mathfrak{p}}=\mathbb{I}_{\mathfrak{p}}^{*}$.

LEMMA 6.9. The two choices of $\mathcal{R}$ given in the proof of Proposition 6.8 satisfy Conditions (1)-(3) given in Definition 6.5.

Proof. Let $\mathcal{R}$ be the first choice (6.8.1) in the proof of 6.8. Condition (1) of Definition 6.5 is direct from the definition of $\left.\mathcal{R}_{W_{\varphi, \eta}}=\right] W_{\varphi, \eta}^{\text {gen }}\left[{ }^{\prime}\right.$ in Definition 5.6. For Condition (2), we distinguish the cases of $W_{\varphi, \eta}$.

- If $W_{\varphi, \eta}$ is a stratum of codimension 1 as in Case 2(a) of Definition 5.6, we have ]$W_{\varphi, \eta}^{\text {gen }}\left[^{\prime}=\right] W_{\varphi, \eta}^{\text {gen }}[$. We may take any $\epsilon, \delta \in(0,1)$.

- If $W_{\varphi, \eta}$ is in Case 2(b) of Definition 5.6, we take $\epsilon=\sum_{i=1}^{f_{\beta}-1} \frac{1}{p^{i}}$ (and there is no need for $\delta$ ).

- If $W_{\varphi, \eta}$ is in Case 2(c) of Definition 5.6, it follows from Proposition 5.15(1) that we can take $\delta=\epsilon=\delta_{j}$.

For Condition (3), we prove first that $\left.\operatorname{sp}^{-1}(\bar{Q}) \cap \mathcal{R}_{W_{\varphi, \eta}}=\operatorname{sp}^{-1}(\bar{Q}) \cap\right] W_{\varphi, \eta}^{\text {gen }}\left[{ }^{\prime}\right.$ is connected for any nowhere-étale codimension-1 stratum $W_{\varphi, \eta}$. For $W_{\varphi, \eta}$ in Case 2(a) of 5.6, we have $\left.\mathrm{sp}^{-1}(\bar{Q}) \cap\right] W_{\varphi, \eta}^{\mathrm{gen}}\left[\left[^{\prime}=\mathrm{sp}^{-1}(\bar{Q})\right.\right.$, which is clearly connected. If $W_{\varphi, \eta}$ is in Case 2(b), we have

$$
\left.\operatorname{sp}^{-1}(\bar{Q}) \cap\right] W_{\varphi, \eta}^{\mathrm{gen}}\left[^{\prime}=\operatorname{sp}^{-1}(\bar{Q})\left(\sum_{i=1}^{f_{\mathfrak{p}}-1} \frac{1}{p^{i}}, 1\right)_{\beta}\right.
$$


with $\ell(\varphi) \cap \eta=\{\beta\}$, which is also clearly connected. For $W_{\varphi, \eta}$ in Case 2(c), the connectedness of $\left.\operatorname{sp}^{-1}(\bar{Q}) \cap\right] W_{\varphi, \eta}^{\text {gen }}['$ was proved in Proposition 5.15. The second half of (3) is clear by Lemma 5.14.

Consider now the second choice of $\mathcal{R}$ (6.8.2) in the proof of 6.8. Condition (1) is direct from the definition of $] W_{\varphi, \eta}^{\text {gen }}\left[{ }^{\prime}\right.$ for codimension-0 stratum $W_{\varphi, \eta}$. For Condition (2), by 2.1.1 and the discussion for the first collection of $\mathcal{R}$, we may take $\delta=1 / p$ and $\epsilon=1-1 / p$ regardless of the cases of $W_{\varphi, \eta}$. For Condition (3), the only nontrivial part is to prove the connectness of

$$
\begin{aligned}
\operatorname{sp}^{-1}(\bar{Q}) \cap \mathcal{R}_{W_{\varphi, \eta}}: & \left.\left.=\operatorname{sp}^{-1}(\bar{Q}) \cap w_{T_{1}}^{-1}(] W_{w_{T_{1}}(\varphi, \eta)}^{\text {gen }} L^{\prime}\right) \cap w_{T_{2}}^{-1}(] W_{w_{T_{2}}(\varphi, \eta)}^{\text {gen }} L^{\prime}\right) \\
& =\left\{Q \in \operatorname{sp}^{-1}(\bar{Q}): w_{T_{1}}(Q) \in\right] W_{w_{T_{1}}(\varphi, \eta)}^{\text {gen }}{ }^{\prime}, w_{T_{2}}(Q) \\
& \in] W_{w_{T_{2}}(\varphi, \eta)}^{\text {gen }}\left[^{\prime}\right\}
\end{aligned}
$$

for each $\bar{Q} \in W_{\varphi, \eta}^{0}=w_{T_{1}}^{-1}\left(W_{w_{T_{1}}(\varphi, \eta)}^{\text {gen }}\right) \cap w_{T_{2}}^{-1}\left(W_{w_{T_{2}}(\varphi, \eta)}^{\text {gen }}\right)$, where $W_{\varphi, \eta}$ is a stratum of codimension 1. Let $\ell(\varphi) \cap \eta=\{\beta\}$ and $\mathfrak{p} \in \mathbb{S}$ such that $\beta \in \mathbb{B}_{\mathfrak{p}}$. We have several cases.

- $\mathfrak{p} \notin T_{1}$ and $\mathfrak{p} \notin T_{2}$. In this case, the stratum $W_{w_{T_{1}}(\varphi, \eta)}$ and $W_{w_{T_{2}}(\varphi, \eta)}$ are in the same case as classified in Definition 5.6. If $Q=(A, H) \in \operatorname{sp}^{-1}(\bar{Q})$ and $w_{T_{i}}(Q)=\left(A_{i}, H_{i}\right)$ for $i=1,2$, then we have a canonical isomorphism of $p$-divisible groups

$$
A\left[\mathfrak{p}^{\infty}\right] \simeq A_{1}\left[\mathfrak{p}^{\infty}\right] \simeq A_{2}\left[\mathfrak{p}^{\infty}\right]
$$

If both $W_{w_{T_{1}}(\varphi, \eta)}$ and $W_{w_{2}(\varphi, \eta)}$ are both in Case $2(\mathrm{a})$, then $\operatorname{sp}^{-1}(\bar{Q}) \cap \mathcal{R}_{W_{\varphi, \eta}}=$ $\mathrm{sp}^{-1}(\bar{Q})$, which is clearly connected. If both of them are in Case 2(b), we have

$$
\mathrm{sp}^{-1}(\bar{Q}) \cap \mathcal{R}_{W_{\varphi, \eta}}=\operatorname{sp}^{-1}(\bar{Q})\left(\sum_{i=1}^{f_{\mathfrak{p}}-1} \frac{1}{p^{i}}, 1\right)_{\beta},
$$

which is also connected. If both strata are in Case 2(c), using the isomorphisms (6.9.1), we see easily that, for $\left.Q \in \operatorname{sp}^{-1}(\bar{Q}), w_{T_{1}}(Q) \in\right] W_{w_{1}(\varphi, \eta)}^{\text {gen }}\left['\right.$ and $w_{T_{2}}(Q) \in$ ]$W_{w_{T_{2}}(\varphi, \eta)}^{\mathrm{gen}}\left[{ }^{\prime}\right.$ hold if and only if $\left.Q \in\right] W_{\varphi, \eta}^{\mathrm{gen}}\left[{ }^{\prime}\right.$. Hence, we get

$$
\left.\operatorname{sp}^{-1}(\bar{Q}) \cap \mathcal{R}_{W_{\varphi, \eta}}=\operatorname{sp}^{-1}(\bar{Q}) \cap\right] W_{\varphi, \eta}^{\mathrm{gen}}\left[^{\prime},\right.
$$

which is connected by Proposition 5.15.

- $\mathfrak{p} \in T_{1}$ and $\mathfrak{p} \in T_{2}$. We have then

$$
\begin{aligned}
\operatorname{sp}^{-1}(\bar{Q}) & \cap \mathcal{R}_{W_{\varphi, \eta}} \\
= & \left.w_{\mathfrak{p}}^{-1}\left(\operatorname{sp}^{-1}\left(w_{\mathfrak{p}}(\bar{Q})\right) \cap w_{T_{1}-\{\mathfrak{p}\}}^{-1}(] W_{w_{T_{1}}(\varphi, \eta)}^{\text {gen }}\left[^{\prime}\right) \cap w_{T_{2}-\{\mathfrak{p}\}}^{-1}(] W_{w_{T_{2}(\varphi, \eta)}}^{\text {gen }} L^{\prime}\right)\right) .
\end{aligned}
$$


As $w_{\mathfrak{p}}^{-1}$ is an isomorphism, $\operatorname{sp}^{-1}(\bar{Q}) \cap \mathcal{R}_{W_{\varphi, \eta}}$ is connected if and only if $\left.\operatorname{sp}^{-1}\left(w_{\mathfrak{p}}(\bar{Q})\right) \cap w_{T_{1}-\{\mathfrak{p}\}}^{-1}(] W_{w_{T_{1}}(\varphi, \eta)}^{\text {gen }}{ }^{\prime}\right) \cap w_{T_{2}-\{\mathfrak{p}\}}^{-1}(] W_{w_{T_{2}(\varphi, \eta)}}^{\text {gen }}\left[^{\prime}\right)$ is. We conclude thus by the previous case.

- $\mathfrak{p}$ lies in one of $T_{1}$ and $T_{2}$, but not in the other. In this case, exactly one of $W_{w_{T_{1}}(\varphi, \eta)}$ and $W_{w_{T_{2}}(\varphi, \eta)}$ is bad in the sense of Definition 5.5. We may assume thus that $W_{w_{T_{1}}(\varphi, \eta)}$ is bad and that $W_{w_{T_{2}}(\varphi, \eta)}$ is good. We have then

$$
\begin{aligned}
\operatorname{sp}^{-1}(\bar{Q}) \cap \mathcal{R}_{W_{\varphi, \eta}} & =\operatorname{sp}^{-1}(\bar{Q}) \cap w_{T_{1}}^{-1}(] W_{w_{T_{1}}(\varphi, \eta)}^{\mathrm{gen}}\left[^{\prime}\right) \\
& =w_{T_{1}}^{-1}\left(\operatorname{sp}^{-1}\left(w_{T_{1}}(\bar{Q})\right) \cap\right] W_{w_{T_{1}}(\varphi, \eta)}^{\text {gen }}\left[^{\prime}\right)
\end{aligned}
$$

As $w_{T_{1}}^{-1}$ is an isomorphism, the connectivity of $\operatorname{sp}^{-1}(\bar{Q}) \cap \mathcal{R}_{W_{\varphi, \eta}}$ follows from Proposition 5.15.

For any $S \subset \mathbb{S}$, let $\Sigma_{S}=\bigcup_{T \subseteq S} w_{T}^{-1}(\Sigma)$.

Lemma 6.10. Let $\mathfrak{p} \notin S \subset \mathbb{S}$, and let $\mathfrak{n}$ be an ideal of $\mathcal{O}_{L}$ prime to $p$. The following hold true.

(1) Every connected component of $\alpha^{-1}\left(\Sigma_{S}\right)$ contains $\alpha^{-1}\left(\mathrm{sp}^{-1}(W)\right)$, where $W$ is an irreducible component of $W_{\mathbb{B}, \emptyset} \subset \tilde{\bar{Y}}$.

(2) Every connected component of $\pi_{1, \mathfrak{n}}^{-1} \alpha^{-1}\left(\Sigma_{S} \cap w_{\mathfrak{p}}^{-1}\left(\Sigma_{S}\right)\right)$ intersects a region of the form $\pi_{1, \mathfrak{n}}^{-1}\left(\tilde{\mathfrak{Z}}_{\text {rig }}^{|\tau| \leqslant 1} \underline{I}\right)=\pi_{1, \mathfrak{n}}^{-1} \alpha^{-1}\left(\tilde{\mathfrak{Y}}_{\mathrm{rig}}^{|\tau| \leqslant 1} \underline{I}\right)$, where $\underline{I}$ is a multiset of intervals satisfying $I_{\mathfrak{p}}=\mathbb{I}_{\mathfrak{p}}^{*}$ given in Definition 6.1.

Proof. Since $\alpha$ and $\pi_{1, \mathfrak{n}}$ are finite flat maps, it is enough to prove the statements with all instances of $\pi_{1, \mathfrak{n}}^{-1}, \alpha^{-1}$ removed. For part (1), by Proposition 6.8 and in view of the definition of $\Sigma_{S}$, it is enough to prove that, given $T_{1}, T_{2} \subset \mathbb{S}$ with $T_{2}=T_{1} \sqcup\{\mathfrak{p}\}$ for some $\mathfrak{p} \in \mathbb{S}$, every connected component $D$ of $w_{T_{2}}^{-1}(\Sigma)$ intersects $w_{T_{1}}^{-1}(\Sigma)$. As $w_{T_{1}}$ is an automorphism, we have $D=w_{T_{2}}^{-1}\left(D^{\prime}\right)$, where $D^{\prime}$ is a connected component of $\Sigma$. It is, therefore, enough to show that $w_{\mathfrak{p}}^{-1}\left(D^{\prime}\right)$ intersects $\Sigma$. By Proposition $6.8, D^{\prime}$ contains $\operatorname{sp}^{-1}(V)$, where $V$ is an irreducible component of $W_{\mathbb{B},\{\beta\}}$ for some $\beta \in \mathbb{B}_{\mathfrak{p}}$. Therefore, $w_{\mathfrak{p}}^{-1}\left(D^{\prime}\right)$ contains $\operatorname{sp}^{-1}\left(w_{\mathfrak{p}}^{-1}(V)\right)$ which intersects $\Sigma$, as $w_{\mathfrak{p}}^{-1}(V)$ is an irreducible component of $w_{\mathfrak{p}}^{-1}\left(W_{\mathbb{B},\{\beta\}}\right)$, easily seen to be a nowhere-étale stratum of codimension 1.

For part (2), we write

$$
\Sigma_{S} \cap w_{\mathfrak{p}}^{-1}\left(\Sigma_{S}\right)=\bigcup_{T_{1}, T_{2} \subseteq S} w_{T_{1}}^{-1}(\Sigma) \cap w_{T_{2} \cup\{\mathfrak{p}\}}^{-1}(\Sigma) .
$$


By Proposition 6.8, every connected component of every term appearing in the union above intersects a region of the form $\tilde{\mathfrak{Y}}_{\text {rig }}^{|\tau| \leqslant 1} \underline{I}$, where $\underline{I}$ is a multiset of intervals satisfying $I_{\mathfrak{p}}=\mathbb{I}_{\mathfrak{p}}^{*}$. This proves the result.

LEMMA 6.11. Let $\mathfrak{n}$ be an ideal of $\mathcal{O}_{L}$ prime to $p$, and let $\mathfrak{p} \in \mathbb{S}$. Let $\mathcal{W}$ be any of $\alpha^{-1}\left(\Sigma_{S}\right), \alpha^{-1} w_{\mathfrak{p}}^{-1}\left(\Sigma_{S}\right), \tilde{\mathfrak{Z}}_{\text {rig }}^{|\tau| \leqslant 1} \underline{I}$ for any $\underline{I}$. Then, $\pi_{2, \mathfrak{n}}^{-1}(\mathcal{W})=\pi_{1, \mathfrak{n}}^{-1}(\mathcal{W})=\pi_{2, j_{\mathfrak{n}}}^{-1}(\mathcal{W})$ for all $j_{\mathfrak{n}} \in \mathcal{O}_{L} / \mathfrak{n}$.

Proof. All of these regions are defined via the $\mathfrak{p}$-divisible group of the HBAV, and dividing by a subgroup of $A[\mathfrak{n}]$ leaves that $\mathfrak{p}$-divisible group unchanged, as $\mathfrak{p} \backslash \mathfrak{n}$.

Consider a collection $\left\{f_{T}: T \subset \mathbb{S}\right\}$ of elements of $\mathcal{M}_{\underline{k}}^{\dagger}\left(\Gamma_{1}(N p) ; K\right)$, as in Theorem 4.1. Then, by Theorem 5.8, each $f_{T}$ extends analytically to $\alpha^{-1}(\Sigma)$.

THEOREM 6.12. Let the notation be as above. Each $f_{T}$ extends from $\alpha^{-1}(\Sigma)$ to

$$
\alpha^{-1}\left(\Sigma_{\mathbb{S}}\right)=\bigcup_{T \subset \mathbb{S}} w_{T}^{-1}\left(\alpha^{-1}(\Sigma)\right)
$$

Proof. We will assume below that the collection of characters is ramified at all $\mathfrak{p} \in \mathbb{S}$ (as explained in Condition (1) in the statement of Theorem 4.1). The case where the collection of characters is unramified at all $\mathfrak{p} \in \mathbb{S}$ is proved in [19], and the intermediate cases can be proved by a straightforward mixing of the argument in [19] and the argument below.

By symmetry, it is enough to prove the theorem for $f_{\emptyset}$. We prove by induction that, if $S \subseteq \mathbb{S}$, then all elements of $\mathcal{F}_{S}:=\left\{f_{T}: T \subseteq \mathbb{S}-S\right\}$ can be extended to $\alpha^{-1}\left(\Sigma_{S}\right)$. We have already proven this for $S=\emptyset$, and we need to prove it for $S=\mathbb{S}$. Assuming that this claim holds for $S \subset \mathbb{S}$, we show that it holds for $S \cup\{\mathfrak{p}\}$, where $\mathfrak{p} \in \mathbb{S}-S$.

As in the proof of [4, Theorem 10.1], we would ideally like to glue $f_{T}$ and $w_{\mathfrak{p}}\left(f_{T \cup\{\mathfrak{p}\}}\right)$ to extend $f_{T}$ from $\alpha^{-1}\left(\Sigma_{S}\right)$ to $\alpha^{-1}\left(\Sigma_{S \cup\{\mathfrak{p}\}}\right)$. However, two complications arise. First, it turns out that $w_{\mathfrak{p}}\left(f_{T \cup\{\mathfrak{p}\}}\right)$ is no longer equal to $f_{T}$, since, unlike the classical case, the characters $\chi_{T}$ will have conductor away from $p$ (essentially due to the existence of totally positive units). This necessitates the introduction of the auxiliary variety $\tilde{\mathfrak{Z}}_{\text {rig }}^{\mathfrak{n}}$ over which a candidate for the replacement of $f_{T \cup\{\mathfrak{p}\}}$ lives. Second, unlike in the elliptic case, the $f_{T}$ are not defined over the entire nonordinary locus, and this causes complications in the gluing process in that the overlap regions will have several connected components that need to be controlled. This issue can be resolved using our detailed study of the connectivity properties of certain regions on $\tilde{\mathfrak{Y}}_{\text {rig }}$ in Section 6. For this to 
work, it is crucial that our analytic continuation result, Theorem 5.8, provides a 'big enough' domain of automatic analytic continuation for the $f_{T}$.

For any fractional ideal $\mathfrak{a}$ of $\mathcal{O}_{L}$, we consider a cusp $T a_{\mathfrak{a}}$ of $\tilde{\mathfrak{Z}}_{\text {rig }}$ which belongs to the connected component $\tilde{\mathfrak{Z}}_{\text {rig, } \mathfrak{a}}$ of $\tilde{\mathfrak{Z}}_{\text {rig }}$, where the polarization module is isomorphic to $\left(\mathfrak{a}, \mathfrak{a}^{+}\right)$as a module with a notion of positivity:

$$
T a_{\mathfrak{a}}=\left(\underline{\underline{\left(\mathbb{G}_{m} \otimes \mathfrak{d}_{L}^{-1}\right) / q^{\mathfrak{a}^{-1}}}},\left[\zeta_{\mathfrak{p}}\right]\right),
$$

the notation being as at the beginning of this section. We assume that the subgroup generated by the level $N p$ structure is the image of $\left(\mathbb{G}_{m} \otimes \mathfrak{d}_{L}^{-1}\right)[p N]$. Let $\eta^{\underline{k}}$ denote a generator of the sheaf $\omega^{k}$ on the base of $T a_{\mathfrak{a}}$. Let $\mathfrak{p} \notin T \supseteq S$ be as above. For any choice of $\mathfrak{a}$, we write

$$
\begin{aligned}
& f_{T}\left(T a_{\mathfrak{a}}\right)=\sum_{\xi \in\left(\mathfrak{a}^{-1}\right)^{+}} a_{\xi(\mathfrak{a})} q^{\xi} \eta^{\underline{k}}, \\
& f_{T \cup\{\mathfrak{p}\}}\left(T a_{\mathfrak{a}}\right)=\sum_{\xi \in\left(\mathfrak{a}^{-1}\right)^{+}} b_{\xi(\mathfrak{a})} q^{\xi} \eta^{\underline{k}},
\end{aligned}
$$

recalling that they are both normalized in the sense that $c\left(\mathcal{O}_{L}, f_{T}\right)=c\left(\mathcal{O}_{L}\right.$, $\left.f_{T \cup\{\mathfrak{p}\}}\right)=1$. By [39, (2.23)], we have $a_{\xi(\mathfrak{a})}=c\left((\xi) \mathfrak{a}, f_{T}\right)$, and similarly for $b_{\xi(\mathfrak{a})}$.

For simplicity of notation, let us denote $\chi_{T \cup\{\mathfrak{p}\}} / \chi_{T}$ by $\Psi$, and put $\mathcal{O}_{\mathfrak{p}}=\mathcal{O}_{L_{\mathfrak{p}}}$. By the assumptions stated in Theorem 4.1, and since the collection of characters is assumed ramified at $\mathfrak{p}, \Psi$ has conductor $\mathfrak{p n}_{T}$ for some integral ideal $\mathfrak{n}_{T} \mid N$, and $\psi_{T, \mathfrak{p}}=\psi_{T \cup\{\mathfrak{p}\}, \mathfrak{p}}^{-1}=\left.\Psi^{-1}\right|_{\mathcal{O}_{\mathfrak{p}}^{\times}} \bmod 1+\mathfrak{p} \mathcal{O}_{\mathfrak{p}}$ is a nontrivial character of $\left(\mathcal{O}_{L} / \mathfrak{p}\right)^{\times}$ we denote these common characters by $\psi_{\mathfrak{p}}$. We set $\mathfrak{n}:=\mathfrak{n}_{T}$, and let $\psi_{\mathfrak{n}}$ be the character of $\left(\mathcal{O}_{L} / \mathfrak{n}\right)^{\times}$which is obtained as $\left.\Psi^{-1}\right|_{\hat{\mathcal{O}}^{\times}} \bmod \Pi_{\mathfrak{q} \mid \mathfrak{n}}\left(1+\mathfrak{q}^{\text {ord }_{\mathfrak{q}} \mathfrak{n}}\right)$. Let $r_{\mathfrak{p}}$ (respectively, $s_{\mathfrak{p}}$ ) denote the $U_{\mathfrak{p}}$-eigenvalues of $f_{T}$ (respectively, $f_{T \cup\{\mathfrak{p}\}}$ ).

Let $c_{\mathfrak{p}} \in \mathfrak{p}^{-1} \mathfrak{a}^{-1}-\mathfrak{a}^{-1}$ and $c_{\mathfrak{n}} \in \mathfrak{n}^{-1} \mathfrak{a}^{-1}-\bigcup_{\mathfrak{q} \mid \mathfrak{n}} \mathfrak{q} \mathfrak{n}^{-1} \mathfrak{a}^{-1}$ (where $\mathfrak{q}$ runs over prime ideals) be such that $T a_{\mathfrak{a}}^{0}=\left(T a_{\mathfrak{a}}, q^{c_{\mathfrak{p}}},\left[\zeta_{\mathfrak{n}}\right], q^{c_{\mathfrak{n}}}\right)$ is a cusp on $\tilde{\mathfrak{Z}}_{\text {rig }}^{\mathfrak{p}, \mathfrak{n}}$. Fix an isomorphism $\mathcal{O}_{L} / \mathfrak{p} \times \mathcal{O}_{L} / \mathfrak{n} \rightarrow \mathfrak{p}^{-1} \mathfrak{n}^{-1} \mathfrak{a}^{-1} / \mathfrak{a}^{-1}$ such that its restriction to $\mathcal{O}_{L} / \mathfrak{p}$ (respectively, to $\mathcal{O}_{L} / \mathfrak{n}$ ) is given by multiplication by $c_{\mathfrak{p}}$ (respectively, by $c_{\mathfrak{n}}$ ), and denote its inverse by

$$
\left(t_{\mathfrak{p}}, t_{\mathfrak{n}}\right): \mathfrak{p}^{-1} \mathfrak{n}^{-1} \mathfrak{a}^{-1} / \mathfrak{a}^{-1} \rightarrow \mathcal{O}_{L} / \mathfrak{p} \times \mathcal{O}_{L} / \mathfrak{n}
$$

LEMMA 6.13. Let the notation be as above. We have the following.

(1) For any $\xi \in\left(\mathfrak{p}^{-1} \mathfrak{n}^{-1} \mathfrak{a}^{-1}\right)^{+}-\bigcup_{\mathfrak{q} \mid \mathfrak{n} \mathfrak{p}}\left(\mathfrak{q} \mathfrak{p}^{-1} \mathfrak{n}^{-1} \mathfrak{a}^{-1}\right)^{+}$, we have

$$
a_{\xi}(\mathfrak{p n a})=C \psi_{\mathfrak{p}}\left(t_{\mathfrak{p}}(\xi)\right) \psi_{\mathfrak{n}}\left(t_{\mathfrak{n}}(\xi)\right) b_{\xi}(\mathfrak{p n a})
$$

where $C$ is independent of $\xi$.

(2) $a_{\xi(\mathfrak{p n a})}=r_{\mathfrak{p}} a_{\xi(\mathfrak{n a})}, b_{\xi(\mathfrak{p n a})}=s_{\mathfrak{p}} b_{\xi(\mathfrak{n a})}$ for all $\xi \in\left(\mathfrak{n}^{-1} \mathfrak{a}^{-1}\right)^{+}$. 
Proof. Let $\mathfrak{m}$ be a fractional ideal of $L$ prime to $\mathfrak{p n}$ (the conductor of $\Psi$ ). By the weak approximation theorem, there is $\lambda_{\mathfrak{m}} \in \mathbb{A}_{L}^{\times}$such that the ideal generated by $\lambda_{\mathfrak{m}}$ equals $\mathfrak{m}$, and such that, for every prime ideal $\mathfrak{q} \mid \mathfrak{p} \mathfrak{n}$, we have $\left(\lambda_{\mathfrak{m}}\right)_{\mathfrak{q}} \equiv 1 \bmod q^{\operatorname{ord}_{\mathfrak{q}}(\mathfrak{p n})}$. In this proof, we will view $\Psi$ as a character of $\mathbb{A}_{L}^{\times} / L^{\times}$. In particular, we have $\Psi(\mathfrak{m})=\Psi\left(\lambda_{\mathfrak{m}}\right)$ for any $\mathfrak{m}$ prime to $\mathfrak{p n}$, and any choice of $\lambda_{\mathfrak{m}}$ as above.

By assumptions, $(\xi) \mathfrak{p n a}$ is prime to $\mathfrak{p n}$, and the assumptions in the statement of Theorem 4.1 tell us that

$$
a_{\xi}(\mathfrak{p n a})=c\left((\xi) \mathfrak{p n a}, f_{T}\right)=\Psi\left(\lambda_{(\xi) \mathfrak{p n a}}\right) c\left((\xi) \mathfrak{p n a}, f_{T \cup\{\mathfrak{p}\}}\right)=\Psi\left(\lambda_{(\xi) \mathfrak{p n a}}\right) b_{\xi}(\mathfrak{p n a}) .
$$

So, to prove (1), it is enough to show that, for any $\xi$, $\xi^{\prime}$ as in part (1) of the lemma, we have $\psi_{\mathfrak{p}}\left(t_{\mathfrak{p}}\left(\xi^{\prime}\right) t_{\mathfrak{p}}(\xi)^{-1}\right) \psi_{\mathfrak{n}}\left(t_{\mathfrak{n}}\left(\xi^{\prime}\right) t_{\mathfrak{n}}(\xi)^{-1}\right)=\Psi\left(\lambda_{\left(\xi^{\prime} \xi^{-1}\right)}\right)$, noting that $\lambda_{\xi^{-1} \xi^{\prime}}$ makes sense, as $\xi^{-1} \xi^{\prime}$ is prime to $\mathfrak{p n}$ by the choices.

For every place $v$ of $L$, let $i_{v}: L^{\times} \rightarrow L_{v}^{\times} \subset \mathbb{A}_{L}^{\times}$be the natural inclusion. We can write

$$
\begin{aligned}
\Psi\left(\lambda_{\left(\xi^{\prime} \xi^{-1}\right)}\right) & =\Psi\left(\lambda_{\left(\xi^{\prime} \xi^{-1}\right)}\left(\xi^{\prime}\right)^{-1} \xi\right)=\left.\left.\Psi\right|_{\mathcal{O}_{\mathfrak{p}}^{\times}}\left(i_{\mathfrak{p}}\left(\left(\xi^{\prime}\right)^{-1} \xi\right)\right) \Pi_{\mathfrak{q} \mid \mathfrak{n}} \Psi\right|_{\mathcal{O}_{\mathfrak{q}}^{\times}}\left(i_{\mathfrak{q}}\left(\left(\xi^{\prime}\right)^{-1} \xi\right)\right) \\
& =\psi_{\mathfrak{p}}^{-1}\left(\left(\xi^{\prime}\right)^{-1} \xi \bmod 1+\mathfrak{p} \mathcal{O}_{\mathfrak{p}}\right) \psi_{\mathfrak{n}}^{-1}\left(\left(\xi^{\prime}\right)^{-1} \xi \bmod \Pi_{\mathfrak{q} \mid \mathfrak{n}}\left(1+\mathfrak{q}^{\text {ord }_{\mathfrak{q}}(\mathfrak{n})} \mathcal{O}_{\mathfrak{q}}\right)\right)
\end{aligned}
$$

where we have used that $\left(\xi^{\prime}\right)^{-1} \xi$ is totally positive and, hence, $\left.\Psi\right|_{L \otimes_{\mathbb{Q}} \mathbb{R}}$ $\left(\left(\xi^{\prime}\right)^{-1} \xi\right)=1$. To end the proof of part $(1)$, we use $t_{\mathfrak{p}}\left(\xi^{\prime}\right) t_{\mathfrak{p}}(\xi)^{-1} \equiv \xi^{\prime} \xi^{-1}$ $\bmod \mathfrak{p}\left(\right.$ which implies that $\psi_{\mathfrak{p}}\left(t_{\mathfrak{p}}\left(\xi^{\prime}\right) t_{\mathfrak{p}}(\xi)^{-1}\right)=\psi_{\mathfrak{p}}\left(\xi^{\prime} \xi^{-1} \bmod 1+\mathfrak{p} \mathcal{O}_{\mathfrak{p}}\right)$ ), as well as a similar statement at $\mathfrak{n}$.

Part (2) is immediate, since $f_{T}$ and $f_{T \cup\{\mathfrak{p}\}}$ are $U_{\mathfrak{p}}$-eigenforms with eigenvalues $r_{\mathfrak{p}}, s_{\mathfrak{p}}$, respectively.

Let $f:=\pi_{2, \mathfrak{n}}^{*}\left(f_{T}\right)$ and $g:=\sum_{j_{\mathfrak{n}} \in\left(\mathcal{O}_{L} / \mathfrak{n}\right)^{\times}} \psi_{\mathfrak{n}}^{-1}\left(j_{\mathfrak{n}}\right) \operatorname{pr}^{*} \pi_{2, j_{\mathfrak{n}}}^{*}\left(f_{T \cup\{\mathfrak{p}\}}\right)$, which are both defined on $\pi_{1, \mathfrak{n}}^{-1} \alpha^{-1}\left(\Sigma_{S}\right)=\pi_{2, \mathfrak{n}}^{-1} \alpha^{-1}\left(\Sigma_{S}\right)$ (Lemma 6.11).

LEMMA 6.14. We have the following equality over $\pi_{1, \mathfrak{p}}^{-1} \pi_{1, \mathfrak{n}}^{-1} \alpha^{-1}\left(\Sigma_{S}\right) \subset \tilde{\mathfrak{I}}_{\mathrm{rig}}^{\text {p. }}$, :

$$
\sum_{j_{\mathfrak{p}} \in\left(\mathcal{O}_{L} / \mathfrak{p}\right)^{\times}} \psi_{\mathfrak{p}}\left(j_{\mathfrak{p}}\right) \operatorname{pr}^{*} \pi_{2, j_{\mathfrak{p}}}^{*}(f)=C W\left(\psi_{\mathfrak{n}}^{-1}\right)^{-1} W\left(\psi_{p}\right)\left(\operatorname{pr}^{*} \pi_{2, \mathfrak{p}}^{*}(g)-s_{\mathfrak{p}} \pi_{1, \mathfrak{p}}^{*}(g)\right),
$$

where $W($.$) denotes the Gauss sum.$

Proof. By Proposition 5.9, both sides are defined over $\pi_{1, \mathfrak{p}}^{-1} \pi_{1, \mathfrak{n}}^{-1} \alpha^{-1}\left(\Sigma_{S}\right)$. By definitions, we have, for terms appearing on the right-hand side of the equation,

$$
\begin{aligned}
\operatorname{pr}^{*} \pi_{1, \mathfrak{p}}^{*} \pi_{2, j_{\mathfrak{n}}}^{*}\left(f_{T \cup\{\mathfrak{p}\}}\right)\left(T a^{0}\right) & =\operatorname{pr}^{*} f_{T \cup\{\mathfrak{p}\}}\left(\left(\mathbb{G}_{m} \otimes \mathfrak{d}_{L}^{-1}\right) /<q^{\mathfrak{a}^{-1}}, q^{c_{\mathfrak{n}}} \zeta_{\mathfrak{n}}^{j_{\mathfrak{n}}}>,\left[\zeta_{\mathfrak{p}}\right]\right) \\
& =\sum_{\xi \in\left(\mathfrak{n}^{-1} \mathfrak{a}^{-1}\right)^{+}} b_{\xi(\mathfrak{n})} \zeta_{\mathfrak{p}}^{j_{\mathfrak{n}} t_{\mathfrak{n}}(\xi)} q^{\xi} \eta^{\underline{k}}
\end{aligned}
$$




$$
\begin{aligned}
\operatorname{pr}^{*} \pi_{2, \mathfrak{p}}^{*} \pi_{2, j_{\mathfrak{n}}}^{*}\left(f_{T \cup\{\mathfrak{p}\}}\right)\left(T a^{0}\right) & =\operatorname{pr}^{*} f_{T \cup\{\mathfrak{p}\}} \frac{\left(\left(\mathbb{G}_{m} \otimes \mathfrak{d}_{L}^{-1}\right) /<q^{\mathfrak{a}^{-1}}, q^{c_{\mathfrak{p}}}, q^{c_{\mathfrak{n}}} \zeta_{\mathfrak{n}}^{j_{\mathfrak{n}}}>,\left[\zeta_{\mathfrak{p}}\right]\right)}{\frac{b_{\xi(\mathfrak{p n a})} \zeta_{\mathfrak{n}}^{j_{\mathfrak{n}} t_{\mathfrak{n}}(\xi)} q^{\xi} \eta^{\underline{k}}}{}} \\
& =\sum_{\xi \in\left(\mathfrak{p}^{-1} \mathfrak{n}^{-1} \mathfrak{a}^{-1}\right)^{+}} \frac{b}{b}
\end{aligned}
$$

Similarly, for the terms on the left-hand side of the equation, we can write

$$
\begin{aligned}
& \operatorname{pr}^{*} \pi_{2, j_{\mathfrak{p}}}^{*} \pi_{2, \mathfrak{n}}^{*}\left(f_{T}\right)\left(T a^{0}\right)=\operatorname{pr}^{*} f_{T}\left(\left(\mathbb{G}_{m} \otimes \mathfrak{d}_{L}^{-1}\right) /<q^{\mathfrak{a}^{-1}}, q^{c_{\mathfrak{n}}}, q^{c_{\mathfrak{p}}} \zeta_{\mathfrak{p}}^{j_{\mathfrak{p}}}>,\left[\zeta_{\mathfrak{p}}\right]\right) \\
& =\sum_{\xi \in\left(\mathfrak{p}^{-1} \mathfrak{n}^{-1} \mathfrak{a}^{-1}\right)^{+}} a_{\xi(\mathfrak{p n a})} \zeta^{j_{\mathfrak{p}} t_{\mathfrak{p}}(\xi)} q^{\xi} \eta^{\underline{k}} .
\end{aligned}
$$

Using the above calculations, Lemma 6.13, and the fact that the $U_{\mathfrak{q}}$-eigenvalues of the $f_{T}$ are all assumed zero for $\mathfrak{q} \mid N$, it follows easily that the two sides of the desired equation have the same $q$-expansion at the cusp $T a_{\mathfrak{a}}^{0}$. To prove the lemma, it is enough to show that every connected component of $\pi_{1, \mathfrak{p}}^{-1} \pi_{1, \mathfrak{n}}^{-1} \alpha^{-1}\left(\Sigma_{S}\right) \subset \tilde{\mathfrak{Z}}_{\text {rig }}^{\underline{p}, \mathfrak{n}}$ contains such a cusp. Every such connected component maps surjectively to a connected component of $\alpha^{-1}\left(\Sigma_{S}\right)$, since $\pi_{1, \mathfrak{n}}$ and $\pi_{1, \mathfrak{p}}$ are finite flat. The claim now follows from part (1) of Lemma 6.10.

We continue the proof of Theorem 6.12. By the induction assumption, $f_{T}$ extends to $\Sigma_{S}$, and $w_{\mathfrak{p}}\left(f_{T \cup\{\mathfrak{p}\}}\right)$ extends to $w_{\mathfrak{p}}^{-1}\left(\Sigma_{S}\right)$. By Lemma 6.11,

$$
h:=N_{L / \mathbb{Q}}(\mathfrak{p}) r_{\mathfrak{p}} f-C \psi_{\mathfrak{p}}(-1) W\left(\psi_{\mathfrak{p}}\right) W\left(\psi_{\mathfrak{n}}^{-1}\right)^{-1} w_{\mathfrak{p}}(g)
$$

is defined over $\pi_{1, \mathfrak{n}}^{-1}\left(\Sigma_{S} \cap w_{\mathfrak{p}}^{-1}\left(\Sigma_{S}\right)\right)$. By Assumption (1) in Theorem 4.1, $h$ has (nontrivial) character $\psi_{\mathfrak{p}}$ at $\mathfrak{p}$.

In what follows, we will use the shorthand notation $\underline{\underline{A}}$ for the data $\left(\underline{\underline{A}}, P_{\mathfrak{n}}\right.$, $\left.Q_{\mathfrak{n}}\right)$. Let $\underline{I}$ be any multiset of intervals as in Definition 2.2 such that $I_{\mathfrak{p}}=\mathbb{I}_{\mathfrak{p}}^{*}$ and $\mathfrak{Y}_{\text {rig }}^{|\tau| \leqslant 1} \underline{I} \subset \Sigma$. Let $\left(\underline{\underline{A}}, P_{\mathfrak{p}}, Q_{\mathfrak{p}}\right) \in \pi_{1, \mathfrak{p}}^{-1} \pi_{1, \mathfrak{n}}^{-1}\left(\tilde{\mathfrak{Z}}_{\text {rig }}^{|\tau| \leqslant 1} \underline{I}\right)=\pi_{1, \mathfrak{p}}^{-1} \pi_{2, \mathfrak{n}}^{-1}\left(\tilde{\mathfrak{Z}}_{\text {rig }}^{|\tau| \leqslant 1} \underline{I}\right)$ (Lemma 6.11). We write

$$
\begin{aligned}
& N_{L / \mathbb{Q}}(\mathfrak{p}) r_{\mathfrak{p}} f\left(\underline{\underline{\underline{A}}}, Q_{\mathfrak{p}}\right)-\operatorname{pr}^{*} f\left(\underline{\underline{A}} /\left(P_{\mathfrak{p}}\right), \overline{Q_{\mathfrak{p}}}\right) \\
& \quad=\sum_{j_{\mathfrak{p}} \in\left(\mathcal{O}_{L} / \mathfrak{p}\right)^{\times}} \operatorname{pr}^{*} f\left(\underline{\underline{\underline{A}}} /\left(j_{\mathfrak{p}} P_{\mathfrak{p}}+Q_{\mathfrak{p}}\right), \overline{Q_{\mathfrak{p}}}\right) \\
& \quad=\psi_{\mathfrak{p}}(-1) \sum_{j_{\mathfrak{p}} \in\left(\mathcal{O}_{L} / \mathfrak{p}\right)^{\times}} \psi_{\mathfrak{p}}\left(j_{\mathfrak{p}}\right) \operatorname{pr}^{*} f\left(\underline{\underline{\underline{A}}} /\left(j_{\mathfrak{p}} P_{\mathfrak{p}}+Q_{\mathfrak{p}}\right), \overline{P_{\mathfrak{p}}}\right) \\
& \quad=\psi_{\mathfrak{p}}(-1) \sum_{j_{\mathfrak{p}} \in\left(\mathcal{O}_{L} / \mathfrak{p}\right)^{\times}} \psi_{\mathfrak{p}}\left(j_{\mathfrak{p}}\right) \operatorname{pr}^{*} \pi_{2, j_{\mathfrak{p}}}^{*}(f)\left(\underline{\underline{\underline{A}}}, P_{\mathfrak{p}}, Q_{\mathfrak{p}}\right) \\
& \quad=C_{0}\left(\operatorname{pr}^{*} g \underline{\left.\left.\underline{\underline{A}} /\left(Q_{\mathfrak{p}}\right), \overline{P_{\mathfrak{p}}}\right)-s_{\mathfrak{p}} g\left(\underline{\underline{A}}, P_{\mathfrak{p}}\right)\right),}\right.
\end{aligned}
$$


where $C_{0}=C \psi_{\mathfrak{p}}(-1) W\left(\psi_{\mathfrak{p}}\right) W\left(\psi_{\mathfrak{n}}^{-1}\right)^{-1}$. In the first equality, we have used the fact that $U_{\mathfrak{p}}(f)=r_{\mathfrak{p}} f$, and, in the last equality, we have used Lemma 6.14. We must note that, by Lemma 6.3, all the terms in the above calculation are well defined.

It follows that, if $R=\left(\underline{\underline{A}}, P_{\mathfrak{p}}, Q_{\mathfrak{p}}\right) \in \pi_{1, \mathfrak{p}}^{-1} \pi_{1, \mathfrak{n}}^{-1}\left(\tilde{\mathfrak{Z}}_{\text {rig }}^{|\tau| \leqslant 1} \underline{I}\right)$, we have

$$
h\left(\underline{\underline{\underline{A}}}, Q_{\mathfrak{q}}\right)=\operatorname{pr}^{*} f\left(\underline{\underline{\underline{A}}} /\left(P_{\mathfrak{p}}\right), \bar{Q}_{\mathfrak{p}}\right)-C_{0} s_{\mathfrak{p}} g\left(\underline{\underline{\underline{A}}}, P_{\mathfrak{p}}\right) .
$$

For $j_{\mathfrak{p}} \in\left(\mathcal{O}_{L} / \mathfrak{p}\right)^{\times}$, let $j_{\mathfrak{p}}^{*}$ denote its inverse. Lemma 6.3 implies that both points $R_{1}=\left(\underline{\underline{A}}, j_{\mathfrak{p}}^{*} P_{\mathfrak{p}}-Q_{\mathfrak{p}}, P_{\mathfrak{p}}\right)$ and $R_{2}=\left(\underline{\underline{A}}, P_{\mathfrak{p}}-j_{\mathfrak{p}} Q_{\mathfrak{p}}, Q_{\mathfrak{p}}\right)$ belong to $\pi_{1, \mathfrak{p}}^{-1} \pi_{1, \mathfrak{n}}^{-1}\left(\tilde{\mathfrak{Z}}_{\text {rig }}^{|\tau| \leqslant 1} \underline{\bar{I}}\right)$. Applying the above equality to $\bar{R}_{1}$ and $R_{2}$, we deduce that

$$
h\left(\underline{\underline{\underline{A}}}, P_{\mathfrak{p}}\right)=\psi_{\mathfrak{p}}\left(j_{\mathfrak{p}}\right) h\left(\underline{\underline{\underline{A}}}, Q_{\mathfrak{p}}\right),
$$

for any $\left(\underline{\underline{A}}, P_{\mathfrak{p}}, Q_{\mathfrak{p}}\right) \in \pi_{1, \mathfrak{p}}^{-1} \pi_{1, \mathfrak{n}}^{-1}\left(\tilde{\mathfrak{Z}}_{\text {rig }}^{|\tau| \leqslant 1} \underline{I}\right)$. Since $\psi_{\mathfrak{p}}$ is nontrivial, it follows that $h=0$ on $\pi_{1, \mathfrak{n}}^{-1}\left(\tilde{\mathfrak{Z}}_{\text {rig }}^{|\tau| \leqslant 1} \underline{I}\right)$. Choosing $\underline{I}$ as in part (2) of Lemma 6.10 allows us to deduce that $h=0$ on the bigger domain $\pi_{1, \mathfrak{n}}^{-1} \alpha^{-1}\left(\Sigma_{S} \cap w_{\mathfrak{p}}^{-1}\left(\Sigma_{S}\right)\right)=$ $\pi_{2, \mathfrak{n}}^{-1} \alpha^{-1}\left(\Sigma_{S} \cap w_{\mathfrak{p}}^{-1}\left(\Sigma_{S}\right)\right)$ (Lemma 6.11). Therefore, $N_{L / \mathbb{Q}}(\mathfrak{p}) r_{\mathfrak{p}} f$ defined on $\pi_{2, \mathfrak{n}}^{-1} \alpha^{-1}\left(\Sigma_{S}\right)$ and $C_{0} w_{\mathfrak{p}}(g)$ defined on $w_{\mathfrak{p}}^{-1} \pi_{2, \mathfrak{n}}^{-1} \alpha^{-1}\left(\Sigma_{S}\right)$ glue to extend $f$ to $\pi_{2, \mathfrak{n}}^{-1} \alpha^{-1}\left(\Sigma_{S} \cup w_{\mathfrak{p}}^{-1}\left(\Sigma_{S}\right)\right)=\pi_{2, \mathfrak{n}}^{-1} \alpha^{-1}\left(\Sigma_{S \cup\{\mathfrak{p}\}}\right)$. Since $f=\operatorname{pr}^{*} \pi_{2, \mathfrak{n}}^{*}\left(f_{T}\right)$, it follows that $f_{T}$ can be analytically extended to $\alpha^{-1}\left(\Sigma_{S \cup\{\mathfrak{p}\}}\right)$. This end the proof.

\section{Further analytic continuation}

The aim of this section is to show that the overconvergent modular forms obtained in previous sections can be further analytically continued to the entire Hilbert modular variety; that is, they are classical.

THEOREM 7.1. Let $f$ be an overconvergent Hilbert modular form of level $\Gamma_{1}(N p)$ and weight $\underline{k}$. Assume that $f$ extends analytically to $\alpha^{-1}\left(\Sigma_{\mathbb{S}}\right)$, where

$$
\Sigma_{\mathbb{S}}=\bigcup_{T \subset \mathbb{S}} w_{T}^{-1}(\Sigma)
$$

Then, $f$ is classical.

Using Theorems 6.12 and 7.1, one immediately deduces Theorem 4.1. In the following, we prove Theorem 7.1.

LEMMA 7.2 (Rigid Koecher principle). Let $V$ be an admissible formal scheme over $\mathcal{O}_{K}$, and $\mathscr{F}$ a locally free $\mathcal{O}_{V}$-module of finite rank. Let $V_{\text {rig }}$ be the rigid 
generic fiber of $V$, and let $\mathscr{F}_{\text {rig }}$ be the rigid analytification of $\mathscr{F}$. Suppose that the special fiber $V_{0}$ of $V$ satisfies the $S_{2}$-condition. If $U_{0} \subset V_{0}$ is an open subset with complement of codimension $\geqslant 2$, then the natural restriction map

$$
H^{0}\left(V_{\text {rig }}, \mathscr{F}_{\text {rig }}\right) \stackrel{\sim}{\rightarrow} H^{0}\left(\mathrm{sp}^{-1}\left(U_{0}\right), \mathscr{F}_{\text {rig }}\right)
$$

is an isomorphism.

This lemma is a classical result in rigid analytic geometry. For a proof, see [43, A.3].

Proof of Theorem 7.1. Let $\alpha_{*}\left(\omega^{\underline{k}}\right)$ be the push-forward of the sheaf $\underline{\omega}^{\underline{k}}$ on $\mathfrak{Z}$ via the finite flat map $\alpha: \tilde{\mathfrak{Z}}_{\text {rig }} \rightarrow \tilde{\mathfrak{Y}}_{\text {rig }}$. For any admissible open subset $U \subset \tilde{\mathfrak{Y}}_{\text {rig }}$, we can identify $H^{0}\left(\alpha^{-1}(U), \omega^{\underline{k}}\right)$ with $H^{0}\left(U, \alpha_{*}\left(\omega^{\underline{k}}\right)\right)$. By the rigid GAGA, we just need to prove that $f$, which is a priori a section of $\alpha_{*} \underline{\omega}^{\underline{k}}$ defined over $\Sigma_{\mathbb{S}}$, extends analytically to $\tilde{\mathfrak{Y}}_{\text {rig. }}$. If $T \subset \mathbb{S}$ is a subset, we put $\mathfrak{t}(T)=\prod_{\mathfrak{p} \in T} \mathfrak{p}$ and $\mathfrak{t}^{*}(T)=(p) / \mathfrak{t}$. We have $w_{T}^{-1}\left(\underline{\operatorname{deg}}^{-1}(\underline{1})\right)=\underline{\operatorname{deg}}^{-1}\left(\underline{1}_{t^{*}(T)}\right)$, where $\underline{1}_{t^{*}(T)} \in[0,1]^{\mathbb{B}}$ is the vector with $\beta$-component equal to 1 if $\bar{\beta} \in \mathbb{B}_{\mathfrak{t}^{*}(T)}=\cup_{\mathfrak{p} \mid \mathfrak{t}^{*}(T)} \mathbb{B}_{\mathfrak{p}}$, and 0 otherwise. Since $\Sigma$ contains $\operatorname{deg}^{-1}(\underline{1})$ by definition, the region $\bigcup_{T \subset \mathbb{S}} w_{T}^{-1}\left(\underline{\operatorname{deg}^{-1}}(\underline{1})\right)$ is contained in $\Sigma_{\mathbb{S}}$. As $\overline{\mathfrak{Y}_{\text {rig }}}$ together with $\left\{w_{T}^{-1}\left(\underline{\operatorname{deg}^{-1}}(\underline{1})\right): T \subset \mathbb{S}\right\}$ form an admissible cover of $\tilde{\mathfrak{Y}}_{\text {rig }}$, it suffices to show that $f$ can be extended analytically to $\mathfrak{Y}_{\text {rig. }}$. The existence of an integral model $\mathfrak{Z}$ of $Z_{K}$ finite flat over $\mathfrak{Y}$ implies that the sheaf $\alpha_{*}\left(\underline{\omega}^{k}\right)$ over $\mathfrak{Y}_{\text {rig }}$ is the rigidification of the locally free $\mathcal{O}_{\mathfrak{Y}}$-module $\underline{\omega}^{\underline{k}} \otimes_{\mathcal{O}_{\mathfrak{Y}}} \alpha_{*}\left(\overline{\mathcal{O}_{\mathfrak{Z}}}\right)$. Therefore, by the rigid Koecher principle 7.2, it suffices to show that there exists a closed subset $\bar{V} \subset \bar{Y}$ of codimension $\geqslant 2$, such that $\Sigma_{\mathbb{S}}$ contains the tube $\mathrm{sp}^{-1}(\bar{Y}-\bar{V})$.

For $\mathfrak{p} \in \mathbb{S}$, let $w_{\mathfrak{p}}$ be the automorphism of $[0,1]^{\mathbb{B}}$ given by $\underline{a} \mapsto \underline{b}$ with $b_{\beta}=1-a_{\beta}$ for $\beta \in \mathbb{B}_{\mathfrak{p}}$ and $b_{\beta}=a_{\beta}$ otherwise. For a subset $S \subset \mathbb{S}$, we define $w_{S}$ to be the composite of the $w_{\mathfrak{p}}$ with $\mathfrak{p} \in S$, and $w=w_{\mathbb{B}}$. Let $\underline{x}$ be a vertex point of the hypercube $[0,1]^{\mathbb{B}}$, and let $W_{\underline{x}}$ be the corresponding stratum of codimension 0 . We define

$$
\begin{gathered}
T_{0}=\left\{\mathfrak{p} \in \mathbb{S}: x_{\beta}=0, \forall \beta \in \mathbb{B}_{\mathfrak{p}}\right\}, \\
T_{1}=\left\{\mathfrak{p} \in \mathbb{S}: x_{\beta}=1, \forall \beta \in \mathbb{B}_{\mathfrak{p}}\right\}, \\
T_{2}=\mathbb{B} \backslash\left(T_{0} \cup T_{1}\right) .
\end{gathered}
$$

It is immediate that both $W_{w_{T_{0}}(x)}$ and $W_{w_{T_{0} \cup T_{2}}(x)}$ are nowhere étale (see 5.4 for this notion). Therefore, by definition, $\Sigma$ contains $\operatorname{sp}^{-1}\left(W_{w_{T_{0}}(\underline{x})}^{\text {gen }} \cup W_{w_{T_{0}} \cup T_{2}(\underline{x})}^{\text {gen }}\right)$; hence, $\Sigma_{\mathbb{S}}$ contains the admissible open $w_{T_{0}}^{-1}\left(\mathrm{sp}^{-1}\left(W_{w_{T_{0}}(\underline{x})}^{\mathrm{gen}}\right)\right) \cup w_{T_{0} \cup T_{2}}^{-1} \mathrm{sp}^{-1}\left(W_{w_{T_{0}} \cup T_{2}(\underline{x})}^{\mathrm{gen}}\right)$, which equals

$$
\operatorname{sp}^{-1}\left(w_{T_{0}}^{-1}\left(W_{\left.w_{T_{0}} \underline{x}\right)}^{\mathrm{gen}}\right) \cup w_{T_{0} \cup T_{2}}^{-1}\left(W_{w_{T_{0} \cup T_{2}}(\underline{x})}^{\mathrm{gen}}\right)\right)
$$


Note that $w_{T_{0}}^{-1}\left(W_{w_{T_{0}} \underline{x}}^{\text {gen }}\right) \cup w_{T_{0} \cup T_{2}}^{-1}\left(W_{w_{T_{0}} \cup T_{2}(\underline{x})}^{\text {gen }}\right)$ is an open subset of $W_{\underline{x}}$. We denote by $V_{\underline{x}}$ its complement in $W_{\underline{x}}$, and by $\bar{V}_{\underline{x}}$ the closed closure of $V_{\underline{x}}$ in $\bar{Y}$. By Proposition 2.8, $V_{\underline{x}}$ has codimension $\geqslant 2$ in $W_{\underline{x}}$, so $\bar{V}_{\underline{x}}$ has codimension $\geqslant 2$ in $\bar{Y}$. Let $\mathbf{a}$ be an edge of $[0,1]^{\mathbb{B}}$, and $W_{\mathbf{a}}$ the corresponding stratum of codimension 1 . There exists a unique $\beta_{0} \in \mathbb{B}$ such that a consists of the points $\underline{a}=\left(a_{\beta}\right) \in[0,1]^{\mathbb{B}}$ with $a_{\beta_{0}} \in(0,1)$ and $a_{\beta} \in\{0,1\}$ for $\beta \neq \beta_{0}$. Let $S_{0} \subset \mathbb{S}$ be such that $a_{\beta}=0$ for all $\beta \in \mathbb{B}_{\mathfrak{p}}$, and $\mathfrak{p}_{0} \in \mathbb{S}$ with $\beta_{0} \in \mathfrak{p}_{0}$. Then both $W_{w_{S_{0}} \text { (a) }}$ and $W_{w_{S_{0} \cup\left\{\mathfrak{p}_{0}\right\}}(\mathbf{a})}$ are nowhere étale. Hence, by definition, $\Sigma_{\mathbb{S}}$ contains the subset

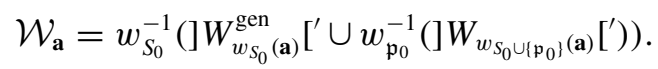

Note that $\mathcal{W}_{\mathbf{a}} \subset \operatorname{sp}^{-1}\left(W_{\mathbf{a}}\right)$. We denote by $W_{w_{s_{0}}(\mathbf{a})}^{\text {sing }}$ the reduced closed subscheme associated with the complement of $W_{w_{S_{0}}(\mathbf{a})}^{\text {gen }}$ in $W_{w_{S_{0}}(\mathbf{a})}$. Then $W_{w_{s_{0}}(\mathbf{a})}^{\text {sing }}$ has codimension $\geqslant 1$ in $W_{w_{S_{0}}(\mathbf{a})}$. We put

$$
V_{\mathbf{a}}=w_{S_{0}}^{-1}\left(W_{w_{0}(\mathbf{a})}^{\text {sing }}\right) \cup w_{S_{0} \cup\left\{\mathfrak{p}_{0}\right\}}^{-1}\left(W_{w_{S_{0} \cup\left\{\mathfrak{p}_{0}\right\}}(\mathbf{a})}^{\text {sing }}\right)
$$

so that

$$
W_{\mathbf{a}}-V_{\mathbf{a}}=w_{S_{0}}^{-1}\left(W_{w_{S_{0}}(\mathbf{a})}^{\mathrm{gen}} \cap w_{\mathfrak{p}_{0}}^{-1}\left(W_{w_{S_{0} \cup\left\{\mathfrak{p}_{0}\right\}}(\mathbf{a})}^{\mathrm{gen}}\right)\right) .
$$

Note that exactly one of $W_{w_{S_{0}} \text { (a) }}$ and $W_{w_{\left.S_{0} \cup \mathfrak{p}_{0}\right\}}(\mathbf{a})}$ is a good codimension-1 stratum in the sense of Definition 5.5. Hence, one has $] W^{\text {gen }}\left[{ }^{\prime}=\right] W^{\text {gen }}[$ for either $W=W_{w_{S_{0}}(\mathbf{a})}$ or $W=W_{w_{S_{0} \cup\left\{\mathfrak{p}_{0}\right\}}(\mathbf{a})}$ by Definition 5.6. It follows immediately that $\operatorname{sp}^{-1}\left(W_{\mathbf{a}}-V_{\mathbf{a}}\right) \subseteq \mathcal{W}_{\mathbf{a}}$. We let $\bar{V}_{\mathbf{a}}$ be the closure of $V_{\mathbf{a}}$ in $\bar{Y}$. Since $W_{\mathbf{a}}$ has codimension 1 in $\bar{Y}, \bar{V}_{\mathbf{a}}$ has codimension $\geqslant 2$ in $\bar{Y}$. Finally, we set

$$
\bar{V}=\left(\bigcup_{\underline{x}} \bar{V}_{\underline{x}}\right) \cup\left(\bigcup_{\mathbf{a}} \bar{V}_{\mathbf{a}}\right) \cup\left(\bigcup_{\operatorname{codim}(W) \geqslant 2} W\right),
$$

where $\underline{x}$ runs through all the vertexes of $[0,1]^{\mathbb{B}}$, a though all the edges, and $W$ through all the strata of codimension $\geqslant 2$. This is a closed subset of $\bar{Y}$ of codimension 2 . By the discussion above, we see that $\Sigma_{\mathbb{S}}$ contains the tube $\operatorname{sp}^{-1}(\bar{Y}-\bar{V})$. This finishes the proof of Theorem 7.1.

\section{Residual modularity}

Let $F$ be a totally real field (on which we will put various conditions). In this section, we prove that certain mod- $p$ representations of $G_{F}=\operatorname{Gal}(\overline{\mathbb{Q}} / F)$ are modular.

8.1. Modularity of icosahedral mod-5 representations of $\boldsymbol{G}_{F}$. We begin with a lemma. 
LEMMA 8.2. Let $F$ be a totally real field. Suppose that $\bar{\rho}: G_{F} \rightarrow G L_{2}\left(\overline{\mathbb{F}}_{5}\right)$ is a continuous representation of $G_{F}=\mathrm{Gal}(\overline{\mathbb{Q}} / F)$ satisfying the following:

- $\bar{\rho}$ is totally odd,

- $\bar{\rho}$ has projective image $A_{5}$,

Then, there is a finite soluble totally real extension $F \subset M \subset \overline{\mathbb{Q}}$ and an elliptic curve $E$ over $M$ satisfying the following conditions:

- $\bar{\rho}_{E, 5}: G_{M}=\operatorname{Gal}(\overline{\mathbb{Q}} / M) \rightarrow \operatorname{Aut}(E[5])$ is equivalent to a twist of $\left.\bar{\rho}\right|_{G_{M}}$ by some character,

- $\bar{\rho}_{E, 3}: \operatorname{Gal}\left(\overline{\mathbb{Q}} / M\left(\zeta_{3}\right)\right) \rightarrow \operatorname{Aut}(E[3])$ is absolutely irreducible,

- E has good ordinary reduction at every place of $M$ above 3, and potentially good ordinary reduction at every place of $M$ above 5.

Proof. Suppose that $\left[F\left(\zeta_{5}\right): F\right]=4$. Then the mod-5 cyclotomic character $\epsilon: \operatorname{Gal}\left(F\left(\zeta_{5}\right) / F\right) \rightarrow(\mathbb{Z} / 5 \mathbb{Z})^{\times}$is an isomorphism. Let $F_{1}$ be the unique quadratic extension $F(\sqrt{5})$ in $F\left(\zeta_{5}\right)$ of $F$ corresponding to the index-2 subgroup of $(\mathbb{Z} / 5 \mathbb{Z})^{\times}$.

Following Taylor [42], since the kernel of the projection $S L_{2}\left(\mathbb{F}_{5}\right) \rightarrow P S L_{2}\left(\mathbb{F}_{5}\right)$ is $\{ \pm 1\}$, the obstruction for lifting $\operatorname{Gal}(\bar{F} / F) \rightarrow P S L_{2}\left(\mathbb{F}_{5}\right)$ to $S L_{2}\left(\mathbb{F}_{5}\right)$ lies in $H^{2}(\operatorname{Gal}(\bar{F} / F),\{ \pm 1\})=\operatorname{Br}(F)[2]$, and we shall 'annihilate' the obstruction by quadratic base-changes. Since $F\left(\zeta_{5}\right)$ is totally ramified at 5 , so is $F_{1}$. Hence the image in the local Brauer group of the obstruction at every place of $F_{1}$ at 5 is trivial. One may and will choose $F_{2}$ to be a totally real quadratic extension of $F$ such that all the places of $F$ not dividing 5, at which local images of the obstruction are nontrivial, remain prime; and every place of $F$ above 5 splits completely. Let $L$ be the bi-quadratic extension $F_{1} F_{2}$ of $F$.

Suppose that $\left[F\left(\zeta_{5}\right): F\right]=2$. Choose a totally real quadratic extension $F_{1}$ of $F$ in which every finite place of $F$ not dividing 5 , at which local images of the obstruction are nontrivial, splits completely, while every place above 5 remains prime. Choose a quadratic extension $F_{2}$ of $F$ as above. Let $L=F_{1} F_{2}$.

In either case, the restriction to $\operatorname{Gal}\left(F\left(\zeta_{5}\right) / L\right)$ of the mod-5 cyclotomic character defines an isomorphism to the order-2 subgroup $\{ \pm 1\}$ of $(\mathbb{Z} / 5 \mathbb{Z})^{\times}$.

The image of the obstruction for $\operatorname{Gal}(\bar{F} / L) \rightarrow P S L_{2}\left(\mathbb{F}_{5}\right)$ to $S L_{2}\left(\mathbb{F}_{5}\right)$ therefore lies in $H^{2}(\operatorname{Gal}(\bar{F} / L),\{ \pm 1\})$, and it has local image trivial everywhere except at the infinite places of $L$.

Since the kernel of the square $(\mathbb{Z} / 5 \mathbb{Z})^{\times} \rightarrow\{ \pm 1\}$ is $\{ \pm 1\}$, the obstruction, for lifting the cyclotomic character $\operatorname{Gal}(\bar{F} / L) \rightarrow\{ \pm 1\}$ to a character $\operatorname{Gal}(\bar{F} / L) \rightarrow$ $(\mathbb{Z} / 5 \mathbb{Z})^{\times}$whose square is the cyclotomotic character, lies in $H^{2}(\operatorname{Gal}(\bar{F} / L)$, 
$\{ \pm 1\})$. Evidently, the image of the obstruction in $H^{2}(\operatorname{Gal}(\bar{F} / L),\{ \pm 1\})$ is trivial everywhere except at the infinite places of $L$.

Hence, there is no obstruction for lifting $\operatorname{proj} \bar{\rho}: \operatorname{Gal}(\bar{F} / L) \rightarrow A_{5}$ to $\bar{\rho}_{L}$ : $\operatorname{Gal}(\bar{F} / L) \rightarrow \mathrm{GL}_{2}\left(\mathbb{Z}_{5}\right)$ with its determinant mod-5 cyclotomic character.

Choose, by class-field theory, a finite soluble totally real extension $L^{\prime}$ of $L$ such that $\bar{\rho}_{L}$ is trivial when restricted to the decomposition group of every place in $L^{\prime}$ above 3 and 5 . The construction follows from class field theory; see Lemma 2.2 in [42] of 'Chevalley's lemma', for example. Let $M$ be the Galois closure of $L$ ' over $F$ (note that $M$ is soluble over $F$ ), and let $\bar{\rho}_{M}$ denote the restriction of $\bar{\rho}_{L}$ to $\operatorname{Gal}(\overline{\mathbb{Q}} / M)$.

As in Section 1 of [38], let $Y_{\bar{\rho}_{M}} / M$ (respectively, $X_{\bar{\rho}_{M}} / M$ ) denote the twist of the (respectively, compactified) modular curve $Y_{5}$ (respectively, $X_{5}$ ) with full level-5 structure. As proved in Lemma 1.1 of [38], the 'twist' cohomology class is in fact trivial, and therefore $X_{\bar{\rho}_{M}} \simeq X_{5}$ and $Y_{\bar{\rho}_{M}}$ is isomorphic over $M$ to a Zariski open subset of the projective line. In particular, $Y_{\bar{\rho}_{M}}$ has infinitely many rational points.

Let $Y_{\bar{\rho}_{M}, 0}(3)$ denote the degree four cover over $Y_{\bar{\rho}_{M}}$ which parameterizes the isomorphism classes $\left(E, \phi_{5}, C\right)$ of elliptic curves $E$ equipped with an isomorphism $\phi_{5}: E[5] \simeq \bar{\rho}_{M}$ taking the Weil pairing on $E[5]$ to $\epsilon: \wedge^{2} \bar{\rho}_{M} \rightarrow \mu_{5}$, and a finite flat subgroup scheme $C \subset E[3]$ of order 3 .

Let $Y_{\bar{\rho}_{M} \text {,split }}(3)$ denote the étale cover over $Y_{\bar{\rho}_{M}}$ which parameterizes the isomorphism classes $\left(E, \phi_{5}, C, D\right)$, where $\left(E, \phi_{5}\right)$ is as in $Y_{\bar{\rho}_{M}}$, and where $(C$, $D)$ is an unordered pair of finite flat subgroup schemes of $E[3]$ of order 3 which intersect trivially. Then, it follows from Lemma 12 in [35] that $Y_{\bar{\rho}_{M}, \text { split }}(3)$ and $Y_{\bar{\rho}_{M}, 0}(3)$ have only finitely many rational points.

For every prime $\mathfrak{p}$ of $M$ above 3 (respectively, 5), the elliptic curve $y^{2}=x^{3}+$ $x^{2}-x$ (respectively, a twist of the CM elliptic curve $y^{2}=x^{3}+x$ ) defines an element of $Y_{\bar{\rho}_{M}}\left(M_{\mathfrak{p}}\right)$ with good ordinary reduction, and we let $\mathcal{U}_{\mathfrak{p}} \subset Y_{\bar{\rho}_{M}}\left(M_{\mathfrak{p}}\right)$ denote a (nonempty) open neighbourhood, for the 3-adic (5-adic) topology, of the point, consisting of elliptic curves with good (respectively, potentially) ordinary reduction at $\mathfrak{p}$.

By the Hilbert irreducibility theorem (Theorem 1.3 in [12]; see also Theorem 3.5.7 in [37]), we may then find a rational point in $Y_{\bar{\rho}_{M}}(M)$ which lies in $\mathcal{U}_{\mathfrak{p}}$ for every $\mathfrak{p}$ above 15 and does not lie in the images of $Y_{\bar{\rho}_{M}, 0}(3)(M) \rightarrow Y_{\bar{\rho}_{M}}(M)$ and $Y_{\bar{\rho}_{M} \text {,split }}(3)(M) \rightarrow Y_{\bar{\rho}_{M}}(M)$. The elliptic curve over $M$ corresponding to the rational point is what we are looking for.

THEOREM 8.3. Let $F$ be a totally real field. Let $\bar{\rho}: G_{F} \rightarrow \mathrm{GL}_{2}\left(\overline{\mathbb{F}}_{5}\right)$ be a continuous representation of the absolute Galois group $G_{F}=\mathrm{Gal}(\overline{\mathbb{Q}} / F)$ of $F$ which satisfies the following conditions: 
- $\bar{\rho}$ is totally odd;

- $\bar{\rho}$ has projective image $A_{5}$.

Then $\bar{\rho}$ is modular.

Proof. This can be proved exactly as in [36, Section 2]. Choose an elliptic curve $E$ over a finite soluble totally real extension $M$ of $F$ as in the preceding lemma. Replace $M$ by its finite totally real soluble extension, if necessary, to assume that the mod-3 representation $\bar{\rho}_{E, 3}$ is unramified at every prime of $M$ above 3 . As argued in the proof of Theorem 3.5.5 in [23], one can do this with the absolute irreducibility of $\left.\bar{\rho}_{E, 3}\right|_{\mathrm{Gal}(\overline{\mathbb{Q}} / M)}$ intact.

By the Langlands-Tunnell theorem, there exists a weight-1 cuspidal Hilbert eigenform $f_{1}$ which gives rise to $\bar{\rho}_{E, 3}$. By 3 -adic Hida theory, we may find a cuspidal Hilbert eigenform $f_{2}$ of weight 2 and of level prime to 3 , ordinary at every prime of $M$ above 3 , which gives rise to $\bar{\rho}_{E, 3}$. As $E$ is ordinary at $3, f_{2}$ renders $\rho_{E, 3}: G_{M} \rightarrow G L\left(T_{3} E\right)$ 'strongly residually modular' in the sense of Kisin [23], and it follows from Theorem 3.5.5 in [23] that $T_{3} E$ is modular. By Falting's isogeny theorem, $E$ is therefore modular. As $\bar{\rho}_{E, 5}$ is modular, $\left.\bar{\rho}\right|_{G_{M}}$ is modular. It then follows from Theorem 3.2.1 of [3] that $\bar{\rho}$ is modular.

REMARK 8.4. When the first draft of this paper was written, our theorems about modularity of mod-5 representations of $\operatorname{Gal}(\bar{F} / F)$ came with conditions at 5; they also appeared in our main theorem about the strong Artin conjecture. At that time, [3] was not written up, and, in order to establish modularity of the mod-5 representation $r$ of the absolute Galois group of a totally real field $L$ with the image of $\operatorname{proj}(r)$ being $A_{5}$ (for example $\bar{\rho}_{L}$ in the proof of the lemma above), it was necessary to assume that either $r$ is distinguished (with a view to making appeal to Ramakrishna/Taylor lifting argument), or the kernel of $\operatorname{proj}(r)$ does not fix $L\left(\zeta_{5}\right)$ (with a view to using the Khare-Wintenberger 'finiteness of deformation rings' argument to lift $r$ to a characteristic zero lifting that is modular). The main theorem of Barnet-Lamb, Gee, and Geraghty [3] completely solves this inconvenience for us.

\section{Hida theory and $\boldsymbol{\Lambda}$-adic companion forms}

9.1. Hilbert modular forms. Let $L$ be a totally real field. In the following, we will define Hilbert modular forms as true automorphic forms for the group $\operatorname{Res}_{L / \mathbb{Q}} \mathrm{GL}_{2, F}$. See Remark 3.1 for the relationship between these forms and the geometric Hilbert modular forms defined in Section 3.1.

As before, $\mathcal{O}_{L}$ denotes the integers of $L$, and $\mathfrak{d}_{L}$ the different of $L$. Let $\mathbb{A}_{L}=$ $\mathbb{A}_{L}^{\infty} \times L_{\infty}$ denote the adeles of $L$. By $\infty$, we shall also mean the product of the 
infinite primes of $L$. For an ideal $M$ of $\mathcal{O}_{L}$, let $L_{M}$ denote the strict ray class field of conductor $M \infty$.

Let $U_{1}(M)$ denote the open compact subgroup of matrices $\left(\begin{array}{ll}a & b \\ c & d\end{array}\right) \in$ $\mathrm{GL}_{2}\left(\mathcal{O}_{L} \otimes_{\mathbb{Z}} \widehat{\mathbb{Z}}\right)$ such that $c \equiv 0 \bmod M$, and $d \equiv 1 \bmod M$. Let $C_{L, M}$ denote the strict ray class group mod $M \infty$; that is, $\mathbb{A}_{L}^{\times} / L^{\times}\left(\mathbb{A}_{L}^{\infty, \times} \cap U_{1}(M)\right) L_{\infty}^{+, \times}$. Let $p$ be a rational prime, and fix an algebraic closure $\overline{\mathbb{Q}}_{p}$ and an isomorphism $\overline{\mathbb{Q}}_{p} \rightarrow \mathbb{C}$.

If $k \in \mathbb{Z}$, let $S_{k, w=1}\left(U_{1}(M) ; \mathbb{C}\right)$ denote the $\mathbb{C}$-vector space, in the sense of Hida [16], of parallel weight $k=\sum_{\tau \in \mathrm{Hom}_{\mathbb{Q}}(F, \mathbb{R})} k \tau$ cusp forms of level $U_{1}(M)$. Let $S_{k}\left(U_{1}(M)\right.$ ) (respectively, $S_{k}\left(U_{1}(M) ; R\right.$ ) for a ring $\left.R \subset \overline{\mathbb{Q}}_{p}\right)$ denote the subspace of forms $f$ in $S_{k, w=1}\left(U_{1}(N)\right.$; $\left.\mathbb{C}\right)$ whose Fourier coefficients $c(\mathfrak{n}, f) \in \mathbb{Z}$ (respectively, $R$ ) for all integral ideals $\mathfrak{n}$ of $\mathcal{O}_{L}$. These spaces come equipped with action of $C_{L, M}$ via the diamond operators $\mathfrak{q} \mapsto\langle\mathfrak{q}\rangle$ for a prime $\mathfrak{q} \nmid M, T_{\mathfrak{q}}$ for a prime $\mathfrak{q} \nmid M$, and $U_{\mathfrak{q}}$ for a prime $\mathfrak{q} \mid M$. Let $h_{k}(M)$ denote the sub-Z्Z-algebra of $\operatorname{End}\left(S_{k}\left(U_{1}(M)\right)\right)$ generated over $\mathbb{Z}$ by all these operators. For a prime $\mathfrak{q} \nmid M$, define $S_{\mathfrak{q}}$ by $\left(\mathbf{N}_{L / \mathbb{Q}} \mathfrak{q}\right)^{k-2}\langle\mathfrak{q}\rangle$.

Fix an integer $N$ prime to $p$. For the ring $\mathcal{O}$ of integers of a finite extension $K$ of $\mathbb{Q}_{p}$, let $e$ denote Hida's idempotent [16, Section 3] of ordinary forms. We set

$$
h_{\mathcal{O}}^{0}(N)=\lim _{r} e\left(h_{2}\left(N p^{r}\right) \otimes_{\mathbb{Z}} \mathcal{O}\right)
$$

We have a natural map (induced by the diamond operators)

$$
\langle\rangle: C_{L, N p^{\infty}} \stackrel{\text { def }}{=}{\underset{r}{r}}_{\lim _{L, N p^{r}}}=\mathbb{A}_{L}^{\times} / \overline{L^{\times}\left(\mathbb{A}_{L}^{\infty, \times} \cap U_{1}\left(N p^{\infty}\right)\right) L_{\infty}^{+, \times}} \longrightarrow h_{\mathcal{O}}^{0}(N)^{\times},
$$

where by $\mathbb{A}_{L}^{\infty, \times} \cap U_{1}\left(N p^{\infty}\right)$ we mean the set of elements in $\mathbb{A}_{L}^{\infty, \times} \cap U_{1}(N)$ which are 1 at every prime $\mathfrak{p}$ of $L$ above $p$.

We let $\operatorname{Tor}_{L, N p^{\infty}}$ (respectively, $\operatorname{Fr}_{L, N p^{\infty}}$ ) denote the torsion subgroup (respectively, a maximal $\mathbb{Z}_{p}$ free subgroup of rank $1+\delta$ with $\delta=0$ if the Leopoldt conjecture holds) of $C_{L, N p^{\infty}}$, and let $\Lambda_{\mathcal{O}}$ denote the completed group algebra over $\mathcal{O}$ of $\operatorname{Fr}_{L, N p^{\infty}}$. Note that $h_{\mathcal{O}}^{0}(N)$ is a $\Lambda_{\mathcal{O}}$-module via \langle\rangle . In [16], Hida proves that $h_{\mathcal{O}}^{0}(N)$ is a torsion free module of finite type over $\Lambda_{\mathcal{O}}$.

We denote by

$$
\text { Art }: \mathbb{A}_{L}^{\times} / \overline{L^{\times} L_{\infty}^{+, \times}} \rightarrow \operatorname{Gal}(\bar{L} / L)^{\mathrm{ab}}
$$

the (global) Artin map, normalized compatibly with the local Artin maps which takes uniformizers to arithmetic Frobenius elements. By abuse of notation, we shall let Art also denote the induced homomorphism $C_{L, N p^{\infty}} \rightarrow$ $\operatorname{Gal}\left(L_{N}\left(\mu_{p^{\infty}}\right) / L\right)$. 
Let $\epsilon$ denote the cyclotomic character

$$
\epsilon: \operatorname{Gal}\left(L_{N}\left(\mu_{p^{\infty}}\right) / L\right) \rightarrow \mathbb{Z}_{p}^{\times} \hookrightarrow \overline{\mathbb{Q}}_{p}^{\times} .
$$

We will let $\epsilon^{\text {cyclo }}$ denote the character

$$
G_{L}=\operatorname{Gal}(\bar{L} / L) \rightarrow \operatorname{Gal}(\bar{L} / L)^{\mathrm{ab}} \rightarrow C_{L, N p^{\infty}} \hookrightarrow \mathcal{O}\left[C_{L, N p^{\infty}}\right]^{\times}=\Lambda_{\mathcal{O}}\left[\operatorname{Tor}_{L, N p^{\infty}}\right]^{\times} .
$$

Note that $\mathfrak{q} \mapsto S_{\mathfrak{q}}$ extends to a homomorphism

$$
S: \Lambda_{\mathcal{O}}\left[\operatorname{Tor}_{L, N p^{\infty}}\right] \rightarrow h_{\mathcal{O}}^{0}(N)^{\times} .
$$

If $\mathfrak{m}$ is a maximal ideal of $h_{\mathcal{O}}^{0}(N)$ with residue field $k(\mathfrak{m})$, there is (see Taylor [41], Carayol [7], Wiles [44], and Rogawski and Tunnell [33]) a continuous representation

$$
\bar{\rho}_{\mathfrak{m}}: G_{L} \rightarrow \mathrm{GL}_{2}(k(\mathfrak{m})),
$$

such that, for all prime ideals $\mathfrak{q}$ not dividing $N p$, the representation is unramified at $\mathfrak{q}$ and $\operatorname{tr} \bar{\rho}_{\mathfrak{m}}\left(\operatorname{Frob}_{\mathfrak{q}}\right)=T_{\mathfrak{q}}$.

Let $\mathcal{K}$ be a finite extension of the field of fractions of $\Lambda_{\mathcal{O}}$, and $\mathcal{O}_{\mathcal{K}}$ the integral closure of $\Lambda_{\mathcal{O}}$ in $\mathcal{L}$. We call a $\Lambda_{\mathcal{O}}$-algebra homomorphism

$$
F_{\mathrm{H}}: h_{\mathcal{O}}^{0}(N) \rightarrow \mathcal{O}_{\mathcal{K}}
$$

a $\Lambda$-adic eigenform (' $\mathrm{H}$ ' for Hida). If the unique maximal ideal $\mathfrak{m} \subset h_{\mathcal{O}}^{0}(N)$ above $\operatorname{ker} F_{\mathrm{H}}$ is non-Eisenstein, that is, $\bar{\rho}_{\mathfrak{m}}$ is absolutely irreducible, it follows from results of Nyssen [25] and Rouquier [34] that there is a continuous representation

$$
\rho_{F_{\mathrm{H}}}: G_{L} \rightarrow \mathrm{GL}_{2}\left(h_{\mathcal{O}}^{0}(N)_{\mathfrak{m}}\right) \stackrel{F_{\mathrm{H}}}{\rightarrow} \mathrm{GL}_{2}\left(\mathcal{O}_{\mathcal{K}}\right),
$$

which is unramified for all primes $\mathfrak{q} \nmid N p$, and satisfies $\operatorname{tr} \rho_{F_{\mathrm{H}}}\left(\right.$ Frob $\left._{\mathfrak{q}}\right)=T_{\mathfrak{q}}$, and $\operatorname{det} \rho_{F_{\mathrm{H}}}=S \circ \epsilon^{\text {cyclo }}$. Moreover, it follow from work of Wiles [44] that, for every $\mathfrak{p} \mid p$,

$$
\left.\rho_{F_{\mathrm{H}}}\right|_{D_{\mathfrak{p}}} \sim\left(\begin{array}{cc}
* & * \\
0 & \phi_{\mathfrak{p}}
\end{array}\right),
$$

where $\phi_{\mathfrak{p}}$ is the unramified character of the decomposition group $D_{\mathfrak{p}}$ at $\mathfrak{p}$ sending Frob $_{\mathfrak{p}}$ to $F\left(U_{\mathfrak{p}}\right)$, and the product of the diagonal characters is $\left.\left(F_{\mathrm{H}} \circ S\right) \circ \epsilon^{\text {cyclo }}\right|_{D_{\mathfrak{p}}}$.

9.2. $\Lambda$-adic companion forms. Let $F$ be a totally real field with ring of integers $\mathcal{O}_{F}$. Let $\mathbb{S}_{F}$ be the set of prime ideals of $\mathcal{O}_{F}$ above $p$. Assume that $p$ is unramified in $F$. In the following, we construct a finite totally real soluble extension $L$ of $F$ and $2^{\left|\mathbb{S}_{L}\right|}$ overconvergent Hilbert modular forms on $\operatorname{Res}_{L / \mathbb{Q}} \mathrm{GL}_{2, L}$ whose various twists by characters of finite order give rise to the Galois representation in question. 
THEOREM 9.3. Let $\mathcal{O}$ be the ring of integers of a sufficiently large finite extension of $\mathbb{Q}_{p}$, with maximal ideal $\mathrm{m}$. Let $\rho: \operatorname{Gal}(\bar{F} / F) \rightarrow \mathrm{GL}_{2}(\mathcal{O})$ be a continuous representation satisfying the following.

- $\rho$ is unramified at only finitely many places of $F$;

- if $\bar{\rho} \stackrel{\text { def }}{=}(\rho \bmod \mathfrak{m})$, there exists a cuspidal Hilbert modular eigenform $f$ such that $\rho_{f}$ is potentially Barsotti-Tate and nearly ordinary at every place of $F$ above $p$ and such that $\bar{\rho}_{f} \simeq \bar{\rho}$;

- $\bar{\rho}$ is absolutely irreducible when restricted to $\operatorname{Gal}\left(\bar{F} / F\left(\zeta_{p}\right)\right)$;

- for every place $\mathfrak{p}_{0}$ of $F$ above $p$, the restriction of $\rho$ to the decomposition group at $\mathfrak{p}$ is the direct sum of characters $\alpha_{\mathfrak{p}_{0}}$ and $\beta_{\mathfrak{p}_{0}}$, such that the images of the inertia subgroup at $\mathfrak{p}_{0}$ are both finite, and $\alpha_{\mathfrak{p}_{0}} / \beta_{\mathfrak{p}_{0}} \neq 1 \bmod \mathfrak{m}$.

Then, there exists a finite totally real soluble extension $L$ of $F$ in which every prime of $F$ above $p$ splits completely, a finite set $\mathcal{S}$ of finite places of $L$ prime to $p$ satisfying the following conditions:

- for any subset $T$ of places of $L$ above $p$, there is a character

$$
\chi_{T}: \operatorname{Gal}(\bar{F} / L) \rightarrow \mathcal{O}^{\times}
$$

and a $\Lambda$-adic eigenform for $L$

$$
F_{\mathrm{H}, T}: h_{\mathcal{O}}^{0}(N) \rightarrow \mathcal{O}_{\mathcal{K}},
$$

where $N$ is an integer prime to $p$ and divisible by all the places in $\mathcal{S}, \mathcal{O}_{\mathcal{K}}$ is the integral closure of $\Lambda_{\mathcal{O}}$ in a finite extension $\mathcal{K}$ of $\operatorname{Frac}\left(\Lambda_{\mathcal{O}}\right)$, together with a height-one prime $\wp_{T}$ of $\mathcal{O}_{\mathcal{K}}$ such that

$-\left.\left(\rho_{F_{\mathrm{H}, T}} \bmod \wp_{T}\right) \simeq \rho\right|_{\mathrm{Gal}(\bar{F} / L)} \otimes \chi_{T}^{-1}$.

- Let $\mathfrak{p}$ be a place of L dividing $p$, and $\mathfrak{p}_{0}$ denote the place of $F$ induced by $\mathfrak{p}$ so that $L_{\mathfrak{p}} \cong F_{\mathfrak{p}_{0}}$. Let $\alpha_{\mathfrak{p}}$ and $\beta_{\mathfrak{p}}$ denote the characters of $\operatorname{Gal}\left(\bar{L}_{\mathfrak{p}} / L_{\mathfrak{p}}\right)$ corresponding to $\alpha_{\mathfrak{p}_{0}}$ and $\beta_{\mathfrak{p}_{0}}$. Then $\alpha_{\mathfrak{p}} / \chi_{T}$ (respectively, $\beta_{\mathfrak{p}} / \chi_{T}$ ) is unramified if $\mathfrak{p} \in T$ (respectively, if $\mathfrak{p} \notin T$ ), and we have

$$
\left(F_{\mathrm{H}, T}\left(U_{\mathfrak{p}}\right) \quad \bmod \wp_{T}\right)= \begin{cases}\left(\alpha_{\mathfrak{p}} / \chi_{T}\right)\left(\text { Frob }_{\mathfrak{p}}\right) & \text { if } \mathfrak{p} \in T, \\ \left(\beta_{\mathfrak{p}} / \chi_{T}\right)\left(\text { Frob }_{\mathfrak{p}}\right) & \text { if } \mathfrak{p} \notin T,\end{cases}
$$

where $\operatorname{Frob}_{\mathfrak{p}} \in \operatorname{Gal}\left(\bar{L}_{\mathfrak{p}} / L_{\mathfrak{p}}\right)$ denotes a Frobenius element. 
Proof. As in the proof of [23, Theorem 3.5.5], we may and will choose $L$ to be a finite totally real soluble extension of $F$ such that

- every prime $\mathfrak{p}$ of $F$ above $p$ splits completely in $L$,

- if $\left.\rho_{L} \stackrel{\text { def }}{=} \rho\right|_{\mathrm{Gal}(\bar{F} / L)}$, then $\rho_{L}$ is totally odd, and is ramified precisely at a finite set $\mathcal{S}$ of finite places of $L$ and possibly at places above $p$; at every place in $\mathcal{S}$, the image of the inertia subgroups is unipotent,

- $\bar{\rho}_{L}=\left(\rho_{L} \bmod \mathfrak{m}\right)$ is unramified outside $p$ and is absolutely irreducible when restricted to $L\left(\zeta_{p}\right)$,

- there exists a cuspidal automorphic representation $g=\mathrm{BC}(f)$ of $\mathrm{GL}_{2}\left(\mathbb{A}_{L}\right)$ such that $\bar{\rho}_{g} \sim \bar{\rho}$, and $g$ is nearly ordinary at every place of $L$ above $p$ and is special at every place in $\mathcal{S}$.

By class field theory, one can choose a character $\chi_{T}: \operatorname{Gal}(\bar{F} / L) \rightarrow \mathcal{O}^{\times}$such that, if $\mathfrak{p}$ is a place of $L$ dividing $p$, then $\left.\chi_{T}\right|_{I_{\mathfrak{p}}}=\left.\alpha_{\mathfrak{p}}\right|_{I_{\mathfrak{p}}}$ for $\mathfrak{p} \in T$ and $\left.\chi_{T}\right|_{I_{\mathfrak{p}}}=\left.\beta_{\mathfrak{p}}\right|_{I_{\mathfrak{p}}}$ for $\mathfrak{p} \notin T$. Let $\mathbb{S}_{L}^{u r}$ be the set of primes $\mathfrak{p} \mid p$ such that $\alpha_{\mathfrak{p}} / \beta_{\mathfrak{p}}$ is unramified. We can and do arrange so that the characters $\chi_{T}$ satisfy $\chi_{T}=\chi_{T-\mathbb{S}_{L}^{u r}}$ for all $T$. In other words, if $\mathfrak{p} \in \mathbb{S}_{L}^{u r}-T$, then $\chi_{T}=\chi_{T \cup\{\mathfrak{p}\}}$.

Let $\rho_{T}=\rho_{L} \otimes \chi_{T}^{-1}$ and $\bar{\rho}_{T}=\left(\rho_{T} \bmod \mathfrak{m}\right)$. Since $\rho_{T}$ is $p$-distinguished, we may appeal to the Ramakrishna/Taylor lifting argument (see Gee's proof of Theorem 3.4 of [13]) to construct a potentially Barsotti-Tate $p$-ordinary lifting $\tilde{\rho}_{T}$ of $\bar{\rho}_{T}$ such that

$$
\left.\tilde{\rho}_{T}\right|_{D_{\mathfrak{p}}} \simeq\left(\begin{array}{cc}
* & * \\
0 & \phi_{T}
\end{array}\right)
$$

where $\phi_{T}$ is an unramified lifting of $\left(\alpha_{\mathfrak{p}} / \chi_{T} \bmod \mathfrak{m}\right)$ if $\mathfrak{p} \in T$ and $\left(\beta_{\mathfrak{p}} / \chi_{T} \bmod \mathfrak{m}\right)$ if $\mathfrak{p} \notin T$. It is indeed strongly residually modular in the sense of Kisin, since the twist of $\rho_{g}$ by the Teichmuller lifting of $\bar{\chi}_{T}$ defines a modular lifting of $\bar{\rho}_{T}$, and (since $\bar{\rho}_{T}$ is ordinary) it follows from Jarvis's level lowering result [17] and the Fontaine-Laffaille theory that the twist is ordinary and Barsotti-Tate at every place of $L$ above $p$. It then follows from Theorem 3.5.5 of [23] that there exists a $p$-ordinary Hilbert modular form $g_{T}$ whose associated Galois representation is isomorphic to $\widetilde{\rho}_{T}$.

Let $\mathfrak{m}_{T}$ denote the maximal ideal of $h_{\mathcal{O}}^{0}(N)$ corresponding to $\bar{\rho}_{g_{T}}$; in other words, $\bar{\rho}_{\mathfrak{m}_{T}} \sim \bar{\rho}_{T}$ and $\left(U_{\mathfrak{p}} \bmod \mathfrak{m}_{T}\right)=\left(\alpha_{\mathfrak{p}} / \chi_{T}\right)\left(\right.$ Frob $\left._{\mathfrak{p}}\right)$ for $\mathfrak{p} \in T$ and $\left(U_{\mathfrak{p}} \bmod \mathfrak{m}_{T}\right)$ $=\left(\beta_{\mathfrak{p}} / \chi_{T}\right)\left(\right.$ Frob $\left._{\mathfrak{p}}\right)$ for $\mathfrak{p} \notin T$.

It follows from Section 5 of [36] that there exists a finite extension $\mathcal{K}$ of $\operatorname{Frac}\left(\Lambda_{\mathcal{O}}\right)$, a $\Lambda$-adic eigenform $F_{\mathrm{H}, T}: h_{\mathcal{O}}^{0}(N)_{\mathfrak{m}_{T}} \rightarrow \mathcal{O}_{\mathcal{K}}$ and a height-one prime $\wp_{T}$ of $\mathcal{O}_{\mathcal{K}}$ such that $\left(\rho_{F_{\mathrm{H}, T}} \bmod \wp_{T}\right)$ is isomorphic to $\rho_{T},\left(F_{\mathrm{H}, T}\left(U_{\mathfrak{p}}\right) \bmod \wp_{T}\right)=$ $\left(\alpha_{\mathfrak{p}} / \chi_{T}\right)\left(\right.$ Frob $\left._{\mathfrak{p}}\right)$ for $\mathfrak{p}$ in $T$, and $\left(F_{\mathrm{H}, T}\left(U_{\mathfrak{p}}\right) \bmod \wp_{T}\right)=\left(\beta_{\mathfrak{p}} / \chi_{T}\right)\left(\right.$ Frob $\left._{\mathfrak{p}}\right)$, for $\mathfrak{p} \notin T$. 
For every $T \subset \mathbb{S}_{L}$, we define $f_{T}=\left(F_{\mathrm{H}, T} \bmod \wp_{T}\right)$. It is clear that the $f_{T}$ are $p$-adic ordinary eigenforms of parallel weight one, and it is standard that they are indeed overconvergent.

LEMMA 9.4. The assumptions stated in Theorem 4.1 hold for the collection of eigenforms $\left\{f_{T}: T \subset \mathbb{S}_{L}\right\}$, and the collection of Hecke characters $\left\{\chi_{T}: T \subset \mathbb{S}_{L}\right\}$.

Proof. Recall that, by construction, $\rho_{T}:=\left.\rho\right|_{\mathrm{Gal}(\bar{F} / L)} \otimes \chi_{T}^{-1}$ is the Galois representation associated to $f_{T}$ for $T \subseteq \mathbb{S}_{L}$. Let $N$ be an integer prime to $p$ and divisible by the prime-to- $p$ part of the conductor of $\rho_{T}$ for all $T \subset \mathbb{S}_{L}$.

We fix $T \subsetneq \mathbb{S}_{L}$ and $\mathfrak{p} \in \mathbb{S}_{L}-T$. Then, the conductor of $\chi_{T \cup\{\mathfrak{p}\}} / \chi_{T}$ divides $p N$. Indeed, assume first that $\alpha_{\mathfrak{p}} / \beta_{\mathfrak{p}}$ is unramified. By the construction of the $\chi_{T}$ in the proof of Theorem 9.3, we have $\chi_{T \cup\{\mathfrak{p}\}} / \chi_{T}=1$, as desired. Now, assume that $\alpha_{\mathfrak{p}} / \beta_{\mathfrak{p}}$ is ramified. For $\mathfrak{q} \in \mathbb{S}_{L}-\{\mathfrak{p}\}$, let $\mathcal{O}_{\mathfrak{q}}$ denote the ring of integers of $L_{\mathfrak{q}}$. Then we have $\left.\chi_{T \cup\{\mathfrak{p}\}}\right|_{\mathcal{O}_{\mathfrak{q}}^{\times}}=\left.\chi_{T}\right|_{\mathcal{O}_{\mathfrak{q}}^{\times}}$, viewed as characters of $\mathbb{A}_{L}^{\times} / L^{\times}$via class field theory, because, by construction, they are either both $\left.\alpha_{\mathfrak{p}}\right|_{\mathcal{O}_{\mathfrak{q}}^{\times}}$or both $\left.\beta_{\mathfrak{p}}\right|_{\mathcal{O}_{\mathfrak{q}}^{\times}}$. Similarly, we see that $\left.\left(\chi_{T \cup\{\mathfrak{p}\}} / \chi_{T}\right)\right|_{\mathcal{O}_{\mathfrak{p}}^{\times}}=\left.\left(\alpha_{\mathfrak{p}} / \beta_{\mathfrak{p}}\right)\right|_{\mathcal{O}_{\mathfrak{p}}^{\times}} \neq 1$. Since $\alpha_{\mathfrak{p}} / \beta_{\mathfrak{p}}$ is tamely ramified, it follows that $\chi_{T \cup\{\mathfrak{p}\}} / \chi_{T}$ has conductor $\mathfrak{p n}_{T}$, for some $\mathfrak{n}_{T} \mid N$.

Let $\psi_{\mathfrak{p}, T}: \mathcal{O}_{\mathfrak{p}}^{\times} \rightarrow \mathcal{O}^{\times}$be the nebentypus character of $f_{T}$ at $\mathfrak{p}$, and let

$$
\Psi_{T}: \mathbb{A}_{L}^{\times} / L^{\times} \rightarrow C_{L, N p^{\infty}} \stackrel{\lfloor)}{\rightarrow} h_{\mathcal{O}}^{0}(N)^{\times} \stackrel{f_{T}}{\rightarrow} \mathcal{O}^{\times}
$$

denote the Diamond character of $f_{T}$, where \langle\rangle is defined in (9.1.1). Then one has $\psi_{\mathfrak{p}, T}=\left.\Psi_{T}^{-1}\right|_{\mathcal{O}_{\mathfrak{p}}^{\times}}$. We will view $\Psi_{T}$ equivalently as a continuous character of $G_{L}$ via class field theory. Then the determinant of $\rho_{T}$ is equal to $\Psi_{T}$, since $f_{T}$ has parallel weight one. From the shape of $\rho$ at $\mathfrak{p}$, it follows that

$$
\left.\Psi_{T}\right|_{\mathcal{O}_{\mathfrak{p}}^{\times}}=\left.\left(\alpha_{\mathfrak{p}} \beta_{\mathfrak{p}} \chi_{T}^{-2}\right)\right|_{\mathcal{O}_{\mathfrak{p}}^{\times}}=\left.\left(\alpha_{\mathfrak{p}} / \beta_{\mathfrak{p}}\right)\right|_{\mathcal{O}_{\mathfrak{p}}^{\times}},
$$

where the second equality used $\left.\chi_{T}\right|_{\mathcal{O}_{\mathfrak{p}}^{\times}}=\left.\beta_{\mathfrak{p}}\right|_{\mathcal{O}_{\mathfrak{p}}^{\times}}$since $\mathfrak{p} \notin T$. Similarly, we have $\left.\Psi_{T \cup\{\mathfrak{p}\}}\right|_{\mathcal{O}_{\mathfrak{p}}^{\times}}=\left.\left(\beta_{\mathfrak{p}} / \alpha_{\mathfrak{p}}\right)\right|_{\mathcal{O}_{\mathfrak{p}}^{\times}}$. It follows that $\left.\Psi_{T}\right|_{\mathcal{O}_{\mathfrak{p}}^{\times}}=\left(\left.\Psi_{T \cup\{\mathfrak{p}\}}\right|_{\mathcal{O}_{\mathfrak{p}}^{\times}}\right)^{-1}=$ $\left.\left(\chi_{T \cup\{\mathfrak{p}\}} / \chi_{T}\right)\right|_{\mathcal{O}_{\mathfrak{p}}^{\times}}$. Therefore, we have

$$
\psi_{\mathfrak{p}, T}^{-1}=\psi_{\mathfrak{p}, T \cup\{\mathfrak{p}\}}=\left.\left(\chi_{T \cup\{\mathfrak{p}\}} / \chi_{T}\right)\right|_{\mathcal{O}_{\mathfrak{p}}^{\times}}
$$

As the conductor of $\left(\chi_{T \cup\{\mathfrak{p}\}} / \chi_{T}\right)$ divides $p N$, we see that all the $f_{T}$ are of level $\Gamma_{1}(p N)$, and the first assumption on in Theorem 4.1 is satisfied.

Let $\mathfrak{l}$ be a prime ideal of $\mathcal{O}_{L}$ not dividing $N p$. Comparing $\operatorname{tr}\left(\rho_{T}\left(\right.\right.$ Frob $\left.\left._{\mathfrak{l}}\right)\right)$ and $\operatorname{tr}\left(\rho_{T \cup\{\mathfrak{p}\}}\left(\right.\right.$ Frob $\left.\left._{\mathfrak{l}}\right)\right)$, it follows that $c\left(f_{T}, \mathfrak{l}\right)=\left(\chi_{T \cup\{\mathfrak{p}\}} / \chi_{T}\right)(\mathfrak{l}) c\left(f_{T \cup\{\mathfrak{p}\}}, \mathfrak{l}\right)$. On the other hand, comparing the determinants of $\rho_{T}\left(\right.$ Frob $\left._{\mathfrak{l}}\right)$ and $\rho_{T \cup\{\mathfrak{p}\}}\left(\right.$ Frob $\left._{\mathfrak{l}}\right)$, we get $\Psi_{T} / \Psi_{T \cup\{\mathfrak{p}\}}=\left(\chi_{T \cup\{\mathfrak{p}\}} / \chi_{T}\right)^{2}$. Using these facts, and the formula for the Hecke 
operators $T_{\mathfrak{l}^{r}}$ in terms of $T_{\mathfrak{l}}$ and $\Psi_{T}$ (for a positive integer $r$ ), it is easy to see that

$$
c\left(f_{T}, \mathfrak{m}\right)=\left(\chi_{T \cup\{\mathfrak{p}\}} / \chi_{T}\right)(\mathfrak{m}) c\left(f_{T \cup\{\mathfrak{p}\}}, \mathfrak{l}\right)
$$

for any ideal $\mathfrak{m} \subset \mathcal{O}_{L}$ which is prime to $N p$. If $\mathfrak{q} \mid p$ is a prime ideal different from $\mathfrak{p}$, then comparing $c\left(\mathfrak{q}, f_{T}\right)$ and $c\left(\mathfrak{q}, f_{T \cup\{\mathfrak{p}\}}\right)$ implies that the above relation also holds for $\mathfrak{m}=\mathfrak{q}$, and hence for all $\mathfrak{m}$ prime to $\mathfrak{p} N$.

Also, using a standard trick, we can assume that $c\left(f_{T}, \mathfrak{q}\right)=0$ for all $T \subset \mathbb{S}_{L}$ and all $\mathfrak{q} \mid N$. This would require increasing $N$, but does not require changing the prime ideal factors of $N$. Finally, the formula for $c\left(\mathfrak{p}, f_{T}\right)$ shows that it is nonzero, and the assumption on the distinguishability of $\rho$ implies that $c\left(\mathfrak{p}, f_{T}\right) \neq c\left(\mathfrak{p}, f_{T \cup\{\mathfrak{p}\}}\right)$ if $\alpha_{\mathfrak{p}} / \beta_{\mathfrak{p}}$ is unramified.

\section{Modularity of Artin representations}

THEOREM 10.1. Let $F$ be a totally real field in which 5 is unramified. Let

$$
\rho: G_{F}=\operatorname{Gal}(\overline{\mathbb{Q}} / F) \rightarrow \mathrm{GL}_{2}(\mathbb{C})
$$

be a continuous representation satisfying the following conditions:

- $\rho$ is totally odd,

- $\rho$ has projective image isomorphic to $A_{5}$,

- for every place $\mathfrak{p}$ of $F$ above 5, the projective image of the decomposition group at $\mathfrak{p}$ has order 2.

Then, there exists a holomorphic Hilbert cuspidal eigenform $f$ of parallel weight 1 such that $\rho$ arises from $f$ in the sense of Rogawski and Tunnell, and, hence, the Artin L-function $L(\rho, s)$ is entire.

Proof. Since $\rho$ is an Artin representation, it has finite image and hence ramifies at only finitely many places of $F$; if we choose an isomorphism $\iota: \overline{\mathbb{Q}}_{5} \rightarrow \mathbb{C}$, so is $\rho_{\iota}:=\iota^{-1} \circ \rho$. Conjugate $\rho_{\iota}$ if necessary to assume that the image of $\rho_{\iota}$ lies in $G L_{2}\left(\overline{\mathbb{Z}}_{5}\right)$, and let $\bar{\rho}_{\iota}$ denote the residual representation $G_{F} \rightarrow G L_{2}\left(\overline{\mathbb{F}}_{5}\right)$.

While $\operatorname{Im}\left(\operatorname{proj} \rho_{l}\right)$ reduces $\bmod 5$ to $\operatorname{Im}\left(\operatorname{proj} \bar{\rho}_{\iota}\right), \operatorname{Im}\left(\operatorname{proj} \rho_{l}\right) \simeq A_{5}$ is simple, and, hence, $\operatorname{Im}\left(\operatorname{proj} \rho_{l}\right) \simeq \operatorname{Im}\left(\operatorname{proj} \bar{\rho}_{l}\right)$. In particular, $\operatorname{Im}\left(\operatorname{proj} \bar{\rho}_{l}\right)$ is isomorphic to $P S L_{2}\left(\mathbb{F}_{5}\right)$. Because of the assumption on the order of the projective image of the decomposition group $D_{\mathfrak{p}}$ at $\mathfrak{p}$ above $5,\left.\rho_{l}\right|_{D_{\mathfrak{p}}}$ is a direct sum of characters $\alpha_{\mathfrak{p}}$ and $\beta_{\mathfrak{p}}$, and the image of $\operatorname{Im}\left(\left.\operatorname{proj} \rho_{\iota}\right|_{D_{\mathfrak{p}}}\right)=\operatorname{Im}\left(\alpha_{\mathfrak{p}} / \beta_{\mathfrak{p}}\right)$ assumes a cyclic subgroup of order 2 in $P S L_{2}\left(\mathbb{F}_{5}\right)$. Furthermore, the image of the wild inertia subgroup at $\mathfrak{p}$ above 5 cannot have order 2 , hence 1 , that is, $\alpha_{\mathfrak{p}} / \beta_{\mathfrak{p}}$ is tamely ramified at $\mathfrak{p}$. 
By Theorems 8.3 and 9.3, there is a finite soluble totally real field extension $L$ of $F$, and $2^{\left|\mathbb{S}_{L}\right|}$ overconvergent cusp eigenforms $\left\{f_{T}\right\}_{T \subset \mathbb{S}_{L}}$ and $2^{\left|\mathbb{S}_{L}\right|}$ characters $\chi_{T}$ of $\operatorname{Gal}(\bar{F} / L)$ of finite order such that $\rho_{f_{T}}=\left.\rho_{l}\right|_{\mathrm{Gal}(\overline{\mathbb{Q}} / L)} \otimes \chi_{T}^{-1}$. By Theorem 4.1, the $f_{T}$ are indeed classical cusp eigenforms. Untwisting, $\left.\rho_{l}\right|_{\mathrm{Gal}(\overline{\mathbb{Q}} / L)}$ arises from a classical weight-one form on $\operatorname{Res}_{L / \mathbb{Q}} \mathrm{GL}_{2, L}$. By automorphic descent, it follows that $\rho_{\iota}$ arises from a classical weight-one form on $\operatorname{Res}_{F / \mathbb{Q}} \mathrm{GL}_{2, F}$.

\section{Acknowledgements}

This program was started when both the first named and the third named authors visited the Institut des Hautes Études Scientifiques (IHES) in September 2011, and we would like to thank the hospitality of IHES heartily. The first named author was partially supported by EPSRC grant EP/H019537/1. The second named author was financially supported by EPSRC whist this paper was prepared. He would like to thank their support and thank King's College London for their hospitality. The third named author was partially supported by China's Recruitment Program of Global Experts, as well as National Center for Mathematics and Interdisciplinary Sciences, Chinese Academy of Sciences.

\section{References}

[1] F. Andreatta and E. Goren, 'Hilbert modular forms: $\bmod p$ and $p$-adic aspects', Mem. Amer. Math. Soc. 173 (2005).

[2] A. Abbes and T. Saito, 'Ramification of local fields with imperfect residue fields', Amer. J. Math. 124 (2002), 879-920.

[3] T. Barnet-Lamb, T. Gee and D. Geraghty, 'Congruences between Hilbert modular forms: constructing ordinary lifts, II', Math. Res. Lett. 18 (2011).

[4] K. Buzzard, 'Analytic continuation of overconvergent eigenforms', J. Amer. Math. Soc. 16 (2003), 29-55.

[5] K. Buzzard, M. Dickinson, N. Shepherd-Barron and R. Taylor, 'On icosahedral Artin representations', Duke Math. J. 109 (2001), 283-318.

[6] K. Buzzard and R. Taylor, 'Companion forms and weight one forms', Ann. of Math. 149 (1999), 905-919.

[7] H. Carayol, 'Sur les représentations $p$-adiques associées aux formes modulaires de Hilbert', Ann. Scient. de l'E.N.S. 19 (1986), 409-468.

[8] C. Chevalley, 'Deux theorems d'arithmetic', J. Soc. Math. Japan 3 (1951), 36-44.

[9] G. Cornell, J. H. Silverman and G. Stevens (Eds.), Modular Forms and Fermat's Last Theorem (Springer-Verlag, 1998).

[10] P. Deligne and K. Ribet, 'Values of abelian L-functions at negative integers over totally real fields', Invent. Math. 59 (1980), 227-286.

[11] F. Diamond, 'On deformation rings and Hecke rings', Ann. of Math. (2) 144 (1) (1996), 137-166.

[12] T. Ekedahl, 'An effective version of Hilbert's irreducibility theorem', in Séminaire de Théorie des Nombres, (Paris, 1988-1989), Progress in Mathematics, 91 (Birkhäuser). 
[13] T. Gee, 'Companion forms over totally real fields, II', Duke Math. J. 136 (2007), 275-284.

[14] E. Goren and F. Oort, 'Stratifications of Hilbert modular varieties', J. Algebraic Geom. 9 (1) (2000), 111-154.

[15] E. Goren and P. Kassaei, 'Canonical subgroups over Hilbert modular varieties', J. für die reine und angewandte Mathematik 670 (2012), 1-63.

[16] H. Hida, 'On $p$-adic Hecke algebras for $G L_{2}$ over totally real fields', Ann. of Math. 128 (1988), 295-384.

[17] F. Jarvis, 'Correspondences on Shimura curves and Mazur's principle above p', Pac. J. Math. 213 (2004), 267-280.

[18] C. Khare and J. P. Wintenberger, 'Serre's modularity conjecture II', Invent. Math. 178 (2009), 505-586.

[19] P. Kassaei, 'Modularity lifting in parallel weight one', J. Amer. Math. Soc. 26 (1) (2013), 199-225.

[20] P. Kassaei, 'Overconvergence, analytic continuation, and classicality: the case of curves', J. für die reine und angewandte Mathematik 631 (2009), 109-139.

[21] N. Katz and B. Mazur, Arithmetic Moduli of Elliptic Curves, Annals of Mathematical Studies, 108 (Princeton University Press, 1985).

[22] M. Kisin and K. F. Lai, 'Overconvergent Hilbert modular forms', Amer. J. Math. 127 (2005), $735-783$.

[23] M. Kisin, 'Moduli of finite flat group schemes and modularity', Ann. of Math. 170 (2009), 1085-1180.

[24] A. Mokrane, 'Quelques remarques sur l'ordinarité', J. Number Theory 73 (1998), 162-181.

[25] L. Nyssen, 'Pseudo-représentations', Math. Ann. 306 (1996), 257-283.

[26] G. Pappas, 'Arithmetic models for Hilbert modular varieties', Comput. Math. 98 (1995), 43-76.

[27] V. Pilloni, 'Formes modulaires $p$-adiques de Hilbert de poids 1', Preprint, 2012.

[28] V. Pilloni, 'Prolongement analytique sur les variétés de Siegel', Duke Math. J. 157 (1) (2011), 167-222.

[29] V. Pilloni and B. Stroh, 'Surconvergence et Classicité: le cas Hilbert', Preprint 2011.

[30] V. Pilloni and B. Stroh, 'Surconvergence, ramification et modularité', Preprint 2013.

[31] M. Rappoport, 'Compactification de l'espace de modules de Hilbert-Blumenthal', Comput. Math. 36 (1978), 255-335.

[32] M. Raynaud, 'Schémas en groupes de type $(p, \ldots, p)$ ', Bull. de la S.M.F. 102 (1974), 241-280.

[33] J. Rogawski and J. Tunnell, 'On Artin $L$-functions associated to Hilbert modular forms of weight one', Invent. Math. 1983 1-42.

[34] R. Rouquier, 'Caractérisation des Caractères et pseudo-Caractères', J. Algebra 180 (1996), 571-586.

[35] K. Rubin, Modularity of mod 5 representations, in [9], p. 463-474.

[36] S. Sasaki, On Artin representations and nearly ordinary Hecke algebras over totally real fields, Preprint.

[37] J. P. Serre, 'Topics in Galois theory', in Research Notes in Mathematics Vol. 1 (Jones and Bartlett Publishers, 1992).

[38] N. Shepherd-Barron and R Taylor, 'Mod 2 and mod 5 icosahedral representations', J. Amer. Math. Soc. 10 (1997), 283-298.

[39] G. Shimura, 'The special values of the zeta functions associated with Hilbert modular forms', Duke Math. J. 45 (3) (1978), 637-679.

[40] H. Stamm, 'On the reduction of the Hilbert-Blumenthal-moduli scheme with $\Gamma_{0}(p)$-level structure', Forum Math. 9 (4) (1990), 405-455. 
[41] R. Taylor, 'On Galois representations associated to Hilbert modular forms', Invent. Math. 98 (1989), 265-280.

[42] R. Taylor, 'On icosahedral Artin representations II', Amer. J. Math. 125 (2003), 549-566.

[43] Y. Tian, Classicality of overconvergent Hilbert eigenforms: case of quadratic residue degree. arXiv:1104.4583.

[44] A. Wiles, 'On ordinary $\lambda$-adic representations associated to modular forms', Invent. Math. 94 (1988), 529-573. 